University of Rhode Island

DigitalCommons@URI

Open Access Dissertations

2014

\title{
INVESTIGATION INTO 3D EARTH STRUCTURE AND SOURCES USING FULL SEISMIC WAVEFORMS
}

Brian M. Covellone

University of Rhode Island, bcovellone@gso.uri.edu

Follow this and additional works at: https://digitalcommons.uri.edu/oa_diss

\section{Recommended Citation}

Covellone, Brian M., "INVESTIGATION INTO 3D EARTH STRUCTURE AND SOURCES USING FULL SEISMIC WAVEFORMS" (2014). Open Access Dissertations. Paper 281.

https://digitalcommons.uri.edu/oa_diss/281

This Dissertation is brought to you for free and open access by DigitalCommons@URI. It has been accepted for inclusion in Open Access Dissertations by an authorized administrator of DigitalCommons@URI. For more information, please contact digitalcommons-group@uri.edu. 
INVESTIGATION INTO 3D EARTH STRUCTURE AND SOURCES USING

FULL SEISMIC WAVEFORMS

BY

BRIAN M. COVELLONE

A DISSERTATION SUBMITTED IN PARTIAL FULFILLMENT OF THE REQUIREMENTS FOR THE DEGREE OF

DOCTOR OF PHILOSOPHY

IN

OCEANOGRAPHY

UNIVERSITY OF RHODE ISLAND 


\title{
DOCTOR OF PHILOSOPHY DISSERTATION \\ $\mathrm{OF}$ \\ BRIAN M. COVELLONE
}

APPROVED:

Dissertation Committee:

\author{
Major Professor Brian Savage \\ Yang Shen \\ Karen M. Fischer \\ Nasser H. Zawia \\ DEAN OF THE GRADUATE SCHOOL
}

UNIVERSITY OF RHODE ISLAND 


\begin{abstract}
Seismograms are the result of the complex interactions between a seismic source, a propagation medium and the seismograph's response. Through the use of 3-dimensional modeling and full seismic waveform data, we quantify and minimize errors associated with the source and propagation medium within our data sets. We compile a new and unique earthquake catalog for the Middle East that is openly available to the public. We quantify the benefits of using a 3-dimensional model relative to a 1-dimensional model to minimizing error in earthquake moment tensors and identify where in the waveform 3-dimensional models outperform 1dimensional models. Two new and unique 3-dimensional seismic wave speed models are computed for the Ontong Java plateau and eastern North American margin. Both models are significant improvements to the resolution of wave speed structures in the crust and upper mantle and provide new information for the evaluation of tectonic features.
\end{abstract}




\section{ACKNOWLEDGMENTS}

I began attending the University of Rhode Island as an undergraduate in the fall of 2003. For those of you who haven't been counting that is a whopping 11 years ago. I had a wonderful run as an undergraduate in the Department of Geosciences and would like to thank each and every member of the faculty and staff past and present; there are far too many people to name here. You started me on this journey and for that I am grateful. Entering graduate school, I went from sharing the tiniest office on campus with 3 other people, to moving into the only office on campus with a balcony; I guess it pays to stick around.

I would first and foremost like to thank my advisor, Brian Savage. Thank you for your non-stop support, guidance, patience, sense of humor and most importantly belief that I could do this. I'll miss spending days and days writing a unnecessarily long code, only to show you and watch as you condense my weeks worth of work into a single line. It was truly a joy working with you and you have taught me more than I ever thought possible. You have been not only a great advisor but also a great friend.

Thank you to my thesis committee, each one of you has made a significant contribution to not only this thesis but to my graduate career and how I think about the Earth. Yang, you brought me into GSO while I was still an undergraduate and introduced me to seismology. Thank you for all of your years of guidance, advisement and support. Chris, your interpretive dances showed me that sometimes the variables in equations actually do have a physical meaning. Thanks for keeping science fun. I'll especially miss the AGU nights at Lefty's O'Doul's and the more recent parties in the red barn. Karen, thank you for letting me participate in your class and for providing me the opportunity to meet a whole new group of people at Brown. Gopu your unique perspective helped me to think differently about how 
to do inverse problems.

Rob, sometimes I think you're the one keeping the whole campus grounded. Thanks for all the distractions and your sense of humor, it was more welcome than you know. Katie you showed me that sometimes, albeit in very rare cases, chemistry is important. Thank you especially for the spectacular raspberry pies! To all of my office mates, residents of 2nd floor Horn Bldg. and past and present members of the SeismoLab group. I would especially like to mention Zhigang, Yong, Xiaofeng, Wei and Haiying, without your help and guidance I would not have gotten through the thousands (millions?) of lines of code I've run through the years. Shifra, for listening to me ramble for 4 years, without you listening and occasionally responding back I would have been talking to the wall more than society deems acceptable.

To the 2007-2013 Boston Celtics, thank you for the distraction 82 (plus 16) nights a year. 2008 was special.

Maryjo, you've picked me up when I was down and always told me to I could do it. Thank you for encouraging me to always do what makes me happy and for helping me keep everything in perspective. Thank you for being my best friend.

Zoey, woof howwwooooo grrr owoooo!

To all of my grandparents, aunts, uncles and cousins, thank you all for the continued support and encouragement. To my grandfather for always telling me I would do something great, I hope this counts.

To my mom and dad. My whole life you have supported and encouraged me to be the best I can be. Thank you for that. 


\section{PREFACE}

The following dissertation examines three distinct geologic settings throughout the Earth. It has been written in manuscript format and is broken into the following three manuscripts:

Manuscript one, "A quantitative comparison between $1 \mathrm{~d}$ and $3 \mathrm{~d}$ source inversion methodologies: Application to the middle east", investigates the difference between synthetically generated seismograms when using a 1-dimensional or 3-dimensional starting model and how these differences effect the quality of results when inverting for earthquake source mechanisms. Abstracts reflecting the progress of this research were presented at the American Geophysical Union (AGU) annual conference in 2008 and 2009, at the Monitoring Research Review (MRR) annually from 2008 through 2011, and at the Geologic Society of America (GSA) international section in Ankara, Turkey in 2010. This chapter was published in the Bulletin of the Seismological Society of America, October 2012.

Manuscript two, titled "Wave speed structure of the Ontong Java Plateau" is a high resolution model of the crust and upper mantle seismic wave speed structure beneath the Ontong Java Plateau. Abstracts of this work were presented at the AGU annual conference in 2011, 2012 and 2013 as well as at a GeoPRISMS planning workshop in 2013. The manuscript is prepared with submission to Earth and Planetary Science Letters anticipated in 2014.

The third manuscript, "Wave speed structure of the eastern North American margin" is a high resolution model of the crust and upper mantle seismic wave speed structure of the eastern margin of North America. 


\section{TABLE OF CONTENTS}

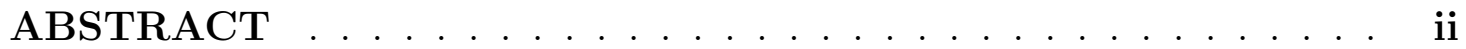

ACKNOWLEDGMENTS ................... iii

PREFACE ........................ . . . . .

TABLE OF CONTENTS .................. . vi

LIST OF TABLES . . . . . . . . . . . . . . . . . viii

TABLE OF FIGURES . . . . . . . . . . . . . . . . . .

CHAPTER 1 A quantitative comparison between $1 \mathrm{~d}$ and $3 \mathrm{~d}$ source inversion methodologies: Application to the middle east .......................... 1

Abstract .......................... 2

Introduction . . . . . . . . . . . . . . . . . 2

Moment Tensor Inversion Methodology . . . . . . . . . . . . 5

Moment Tensor Inversion Results . . . . . . . . . . . . . . 8

Discussion . . . . . . . . . . . . . . . . . 11

Conclusions ....................... . . . 14

Data and Resources . . . . . . . . . . . . . . . 15

Acknowledgments ................... 16

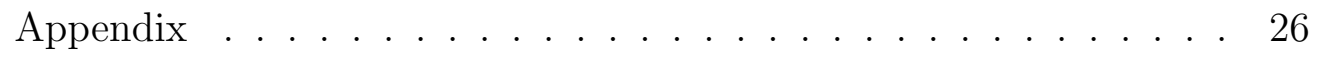

CHAPTER 2 Wave speed structure of the Ontong Java Plateau . 36

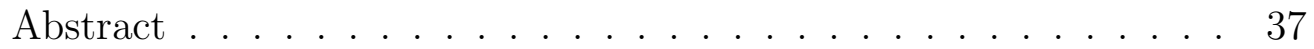

Introduction . . . . . . . . . . . . . . . . . . 37

Methodology ...................... 41

Results ..................... . . 45

Discussion ........................ . . . 49

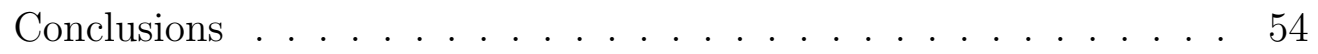


Acknowledgments . . . . . . . . . . . . . . 55

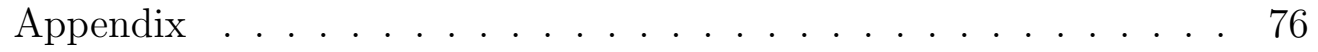

CHAPTER 3 Wave speed structure of the eastern North American margin . . . . . . . . . . . . . . . . 82

Abstract . . . . . . . . . . . . . . 83

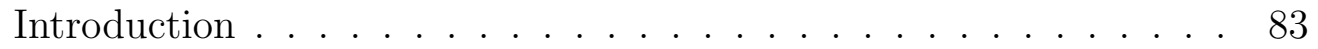

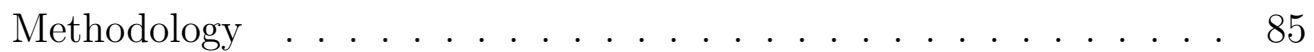

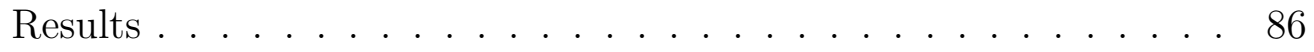

Discussion . . . . . . . . . . . . . . . . . . 89

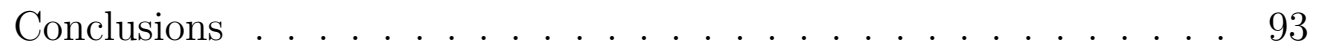




\section{LIST OF TABLES}

TABLE

PAGE

Table 1.1 Comparison between 1D and 3D inversion sets. Standard error on the trend and plunge of the P-axes for all events is calculated from the results of a bootstrap analysis; standard error of the T-axes show a comparable spread and is not reported. Also shown, is the result of calculating the average and standard deviation of $\tau$ from equation 1.3 for each inversion set. A full dataset, mean reduction in variance between the data and misfit between the initial solution, $M_{0}$, solution and our calculated solution is shown. . . . . . . . . . . . . 17

Table 1.2 Earthquake catalog containing 184 event solutions solved for using a 3D moment tensor inversion method Liu et al. (2004). Results are azimuthally weighted, 7-parameter (Mij and depth), zero-trace solutions. Mij components are normalized by dividing by $1 x 10^{17}$ N.m. . . . . . . . . . . . . . . . . 31

Table 2.1 Summary of inversion parameters. . . . . . . . . . . . . . . 67 
Table 2.2 2.5 GPa and $1125^{\circ} \mathrm{C}$. Grossular Garnet (gr), Forsterite (fo), Enstatite (en), Diopside (di), Alpha Quartz (aqz), Almandine (alm), Pyrope (py), Ferrosilite (fs), Hedenbergite (hed), Muscovite (mu), Spinel (sp), Coesite (coe), Jadeite (jd). Jadeite (jd), the sodium bearing clinopyroxene was used in place of Omphacite. Coesite (coe) was used in place of Rutile because it was the closest mineral structure substitute. Phengite and Opaques were combined into Muscovite (mu). References HA04 refers to Hacker and Abers (2004) and Ji03 refers to $\mathrm{Ji}$ et al. (2003). The $m$ prefix signifies the modal abundances have been modified from the published values. . . . . . . . . 79

Table 3.1 Summary of measurement and inversion parameters. Minimum cross correlation coefficient (Min CC), minimum signalto-noise ratio (Min SNR) and maximum phase delay (dT) were used to screen out low quality signals. . . . . . . . . . . . . 95

Table 3.2 A scaled version of the diffusion equation, where $t \approx \frac{\text { radius }^{2}}{\kappa}$, was used to estimate the time needed to completely remove a thermal anomaly given a suite of anomaly radius sizes. Two diffusivity, $\kappa$, values were used, $0.01 \frac{\mathrm{cm}^{2}}{\mathrm{~s}}$ and $0.018 \frac{\mathrm{cm}^{2}}{\mathrm{~s}}$ (Gibert et al., 2003). Diffusion time is given in million years (Ma). . . 95 


\section{LIST OF FIGURES}

FIGURE

PAGE

Figure 1.1 Events (circles) and stations (triangles) used for this study within the Middle East. Inset shows the global distribution of 580 stations used with the 3D S2.9EA wave speed model. Synthetic seismograms were created using the specfem3d software package and calculated for all stations located within the $90^{\circ}$ by $90^{\circ}$ mesh (black box). Major faults and mountain ranges discussed in the paper have been labeled: North Anatolian Fault (NAF); East Anatolian Fault (EAF); Main Recent Fault (MRF); Minab-Zendan-Palami Fault (MZF); Talesh (TL), Alborz (AB), and Kopet-Dagh (KD) Mountain Ranges. . . . . . 18

Figure 1.2 Results of a bootstrap statistical analysis for two single events, a) $1997 / 05 / 13$ and b)2005/02/22. Plotted in the upper left and right are the $\mathrm{P}$-axes on a focal sphere after each inversion $(\mathrm{n}=200)$ using a 1D and 3D model. A tighter cluster of dots indicates a more constrained solution and a smaller standard error on the P-axes of the solution. The histogram shows the distribution of results for the trend $(\mathrm{n}=200)$ for each particular event. . . . . . . . . . . . . . . . . 19 
Figure 1.3 Moment tensor inversion standard error for all events, $\mathrm{n}=195$.

Histograms show the standard error for two frequency bands, 60-125s (a) and (b) and 25-125s (c) and (d), on the trend and plunge. The last bin represents standard errors $>30^{\circ}$. Grey bars represent the use of 3D Kustowski et al. (2008) derived synthetics. White bars represent using 1D model Dziewonski and Anderson (1981) derived synthetics. . . . . . . . . . . . 20

Figure 1.4 Displacement data (black) and synthetic (red) fit for the LHZ component, shown at two stations, II.KURK (left) and IU.KIEV (right), for events 1997/05/13 14:13:45.740 (a-c) and 2005/02/22 02:25:22920 (d-f) filtered at 60-125s and 25-125s and inverted using 1D Dziewonski and Anderson (1981) and 3D Kustowski et al. (2008) wave speed models. Event locations are indicated by (latitude, longitude) beneath the event date. A better fit to the data is seen for complex body waves between 150-450 seconds for II.KURK and 600-900s for station KIEV as well as surface wave dispersion at greater than 800 seconds when using the 3D derived synthetic seismograms (highlighted by the blue box). . . . . . . . . . . . . . . . . . 21

Figure 1.5 Comparison between Global CMT solution (left) and 3D azimuthally weighted 7-parameter solution (right). Our solutions compare well with the initial solution, but variance between the data and synthetic is reduced while fitting more of the data using a 3D model. Percentage represents the variance reduction between our solutions and the Global CMT solution. A majority of events in the catalog (Table S1) see a variance reduction between 5-40\% compared to the Global CMT solution. 22 
Figure 1.6 Original Global CMT depth (Initial Depth) plotted against this study's depths (Revised Depth) from the zero-trace azimuthally weighted solution; a line represents a 1-1 relationship between the two depth solutions. (a) Shows short period (25-125s) solutions which have a tighter fit to the 1-1 line than do (b) longer period (60-125s) solutions. Inset zoomed in to show results at shallow depths. Depth residuals are smaller than symbol size (average residual is less than $2.5 \mathrm{~km}$ ) and therefore not plotted. . . . . . . . . . . . . . . . . 23

Figure 1.7 Histogram showing the percentage double couple (DC) component for short period (25-100 seconds) solutions. A larger \%DC is seen when using 3D derived synthetic seismograms (grey) compared to 1D derived synthetics (white). This result provides evidence that the $\%$ of non-DC component in the solutions is a result of imperfect and poorly resolved Earth structure within the initial velocity models. . . . . . . . . . . 24

Figure 1.8 Earthquake event solutions for the (a) entire dataset and highlighted regions, (b) Hindu Kush and Tian Shan and (c) Iran. Shown are 195 solutions from the 3D zero-trace azimuthally weighted moment tensor inversion. Solutions agree well with regional tectonics and large scale plate motions. . . . . . . 25 
Figure 2.154 Seismic stations (inverted triangles) and 105 earthquake events (circles) used for this study in the Pacific Ocean. Our computational domain is outlined in black. The $4000 \mathrm{~m}$ bathymetric contour of the Ontong Java Plateau (OJP) is outlined in black. Modern plate boundaries are shown with a thin black line. The red arrows show modern Pacific plate motion. Major features have been labeled in black: Central Spreading Ridge (CSR), the Vitiaz Lineament, and Manihiki Plateau. The location of Solomon Islands of Santa Isabel Island (SI), Guadalcanal (GD), and Malaita Island (MI) are also highlighted. . . 67

Figure 2.2 Computational model domain perturbed with $5^{\circ}$ and $7^{\circ}$ sized harmonic positive and negative $5 \%$ wave speed anomalies. Our recovered solution is plotted at three depths into the model. Our recovered resolution is excellent for the region outlined in

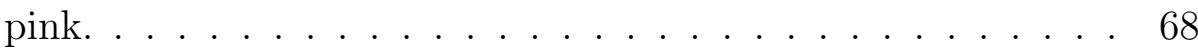

Figure 2.3 (a) Shows the evolution of the $d T$ through iterations; black circles are from AN data, grey circles are from EQ data. The sum of the absolute value of the $d T$ provides a good indication of how the whole data set is improving despite the increase in the number of measurements shown in (b). An increase in the number of measurements through iteration reflects an improvement in fit to the data. . . . . . . . . . . . . . 69 
Figure 2.4 Displacement data (black) and synthetics for iteration 05 (red) and iteration 07 (blue) recorded at station PS.OGS from 5 earthquake events with paths crossing the main fast velocity anomaly beneath the OJP. The $4000 \mathrm{~m}$ bathymetric contour of the OJP is outlined in black. The $d T$ significantly decreases from iteration 05 to iteration 07 , reflecting an improvement in fit to the data. . . . . . . . . . . . . . . . . 70

Figure 2.5 (a-f) Multiple depth slices through the best resolved region of the model (outlined in pink in Figure 2.2. Color scale is absolute $V s$ in $\mathrm{km} / \mathrm{s}$ and remains the same at each depth. . . 71

Figure 2.6 (a) North - South cross section at $151^{\circ}$, a column shaped low wave speed anomaly extends to depths greater than $150 \mathrm{~km}$ on the boundary of the North Bismark/Manus and South Bismark plates. (b) East - West cross section at $-3^{\circ} \mathrm{S}$, showing the fast wave speed feature extending to depths greater than $100 \mathrm{~km}$. (c-d) North - South cross sections at $161^{\circ}$ and $162^{\circ}$, showing the main fast wave speed feature extending to depths greater than $100 \mathrm{~km} . \ldots \ldots \ldots \ldots$. . . . . . . . 72

Figure 2.7 Resolution tests for a $7^{\circ}$ sized fast wave speed anomaly in the location of our observed anomaly. We are able to recover both the shape and amplitude of the fast wave speed anomaly with limited smearing. Also shown is an alternating fast and slow wave speed anomaly simulating the boundary between old, seismically fast lithosphere and young, seismically slow lithosphere. Both fast and slow anomalies are recovered with limited smearing across the boundary. . . . . . . . . . . . 73 
Figure 2.8 The final iteration (ite07) model is shown plotted above two capped model cases, limiting the maximum $V s$ to 4.5 and 4.75 $\mathrm{km} / \mathrm{s}$. For all frequency bands (a- e) the $4.5 \mathrm{~km} / \mathrm{s}$ model is too slow to fit the main surface wave arrival. The $4.75 \mathrm{~km} / \mathrm{s}$ model fits more closely to the observed data. Based on the capping tests, we can confidently identify a wave speed $>4.75$ $\mathrm{km} / \mathrm{s}$ in the fastest portions of our model beneath the OJP. $\quad$ 74

Figure 2.9 The BHT component of our final iteration (ite07) isotropic model is shown plotted above two test cases of anisotropy, a $V s_{v}>V s_{h}$ case as in Beghein et al. (2014) and a $V s_{h}>$ $V s_{v}$ case analogous to adding PREM anisotropy to our model. The $V s_{v}>V s_{h}$ case reveals a transverse component surface wave arrival that is late compared to the data for all frequency bands (a-c). The applied PREM anisotropy case fits the data slightly better than our isotropic model, suggesting that the fast anomaly is weakly anisotropic. . . . . . . . . . . 75

Figure 2.10Empirical Green's Functions (EGF): a) Lines connecting a "virtual" source (red triangle) located at station IU.AFI to each receiver (black triangles). b) EGFs plotted from the "virtual" source to each receiver plotted in (a). EGFs are computed by the cross-correlation of vertical records. Waveforms have been filtered between $0.01-0.02 \mathrm{~Hz}$ and are plotted by distance from the "virtual" source. . . . . . . . . . . . . . 80 
Figure 2.11(a) Shows the main fast wave speed anomaly. Vs values for the constained area shown in a) are plotted as a histogram in b) to illustrate the distribution of shear wave speed at each grid point in the constrained model. The histogram shows that the majority of grid points have a $V s$ value of $<4.75 \mathrm{~km} / \mathrm{s}$. Extreme values, $>4.75$, are from the center of the anomaly. . 81

Figure 3.1203 Seismic stations (inverted triangles) used for this study of the Eastern North American Margin (ENAM). Our computational domain is outlined in black. Modern plate boundaries are outlined in black. . . . . . . . . . . . . . . . . 96

Figure $3.21^{\circ}, 3^{\circ}, 5^{\circ}$ and $7^{\circ}$ sized harmonic pattern of positive and negative $5 \%$ wave speed anomalies. We are able to recover the shape of the $1^{\circ}$ sized anomalies for depths shallower than $100 \mathrm{~km}$ however there is a large decrease in amplitude. Anomalies $3^{\circ}$ and larger are very well resolved in both shape and amplitude for the all of the eastern North American margin and approximately $500 \mathrm{~km}$ offshore. Anomalies of $7^{\circ}$ or larger can be interpreted for much of the Atlantic ocean and Caribbean. . . 97

Figure 3.3 (a) Histogram of the phase delay, $d T$, measured between the data and synthetic waveforms. In grey is iteration 01 , outlined in black is iteration 02. (b) Shows the number of measurements plotted with iteration. An increase in the number of measurements indicates that the model is better able to fit the data and more measurements fall within the minimum acceptance criteria. . . . . . . . . . . . . . . . . . . . . 98 
Figure 3.4 (a-h) Multiple depth slices through the model. The color scale corresponds to absolute $V s$ in $\mathrm{km} / \mathrm{s}$ and remains the same at each depth. Areas of interpretation are outlined in black and discussed in the text. . . . . . . . . . . . . . 99

Figure 3.5 (a-h) Multiple depth slices through the model. The color scale is change in shear wave speed, $d V s$, from the initial model, CUB Ritzwoller et al. (2002), and remains the same at each depth. . . . . . . . . . . . . . . 100

Figure 3.6 (a-b) Cross section through the New England low wave speed region. A seaward shallowing low wave speed anomaly is seen between depths of approximately $50-250 \mathrm{~km}$. (c) Cross section through the low wave speed anomaly offshore of South Carolina and Georgia. A seaward dipping low wave speed anomaly is seen between depths of approximately 35 to greater than $200 \mathrm{~km} . \ldots \ldots \ldots 10 . \ldots \ldots 1$

Figure 3.7 (a-c) Cross section showing the transition between oceanic and continental lithosphere thickness at three locations up the eastern North American margin. The approximate lithosphere asthenosphere boundary is indicated by the dashed black line. A gap in between the thick continental lithosphere and the much thinner oceanic lithosphere is aligned with low wave speed features possibly imaging edge-driven convection cells on the boundary of the continent. . . . . . . . . . . 102 


\title{
CHAPTER 1
}

A quantitative comparison between $1 \mathrm{~d}$ and $3 \mathrm{~d}$ source inversion methodologies: Application to the middle east

by

\author{
Brian M. Covellone ${ }^{1}$; Brian Savage ${ }^{1,2}$
}

Published in the Bulletin of the Seismological Society of America, October 2012

(Covellone and Savage, 2012).

${ }^{1}$ Graduate School of Oceanography, University of Rhode Island, Narragansett, RI 02882

${ }^{2}$ Department of Geosciences, University of Rhode Island, Kingston, RI 02881 


\section{Abstract}

We present a quantitative comparison between seismic moment tensor inversion solutions using 1D and 3D synthetic seismograms, at two frequency bands for events in the Middle East, to assess the effects of 3D models on source studies. Complex geology associated with the active continental convergent margin leads to a scarcity of reliable, available data, necessitating a thorough examination of solution stability and robustness to assure an accurate description of sources with well-characterized source parameters.

Solutions were calculated for 195 events $(\mathrm{Mw}>5.5)$ using a full-waveform moment tensor inversion matching both phase and amplitude. Seismic data processed at two frequency bands compares short and long period performance for 1D and 3D synthetic seismograms. An improvement in fit between data and synthetics is seen using 3D over 1D synthetic seismograms, especially for complex body wave propagation and surface wave dispersion. At short periods, 3D synthetics provide a more robust solution compared to $1 \mathrm{D}$, showing a reduction in error of the source mechanism. Percent double-couple increases with the addition of 3D structure and suggests the percentage of non-double couple component is a result of poorly constrained Earth structure. Event solutions contained in the catalog (Table 1.2) have an average cross-correlation value of 0.87 , with good amplitude ratios, and

are improved (i.e. increased variance reduction) yet consistent with longer period solutions from Global CMT.

\section{Introduction}

The ability to obtain reliable earthquake source solutions is a useful tool to any tectonic interpretation. Source mechanisms prove invaluable in the assessment of plate motions, accurate characterizations of faults, and defining regional stresses. Holt et al. (1991) showed how moment tensors can be related to the seismically re- 
leased strain rate, allowing an estimate of the regional tectonic strain rate. Strain rates calculated from earthquake moment tensors can aid in the evaluation of current GPS velocity fields, as well as an evaluation of hypotheses explaining regional tectonic framework. Obtaining accurate source depths and quantifying their uncertainty are an invaluable source of information providing an additional constraint on plate motions, especially in a region of complex geology with multiple emergent subduction zones, complex fold and thrust belts and thickened crust.

Furthermore, accurate source parameters are necessary to the improvement of 3D Earth models in full-waveform inversion methods. The use of full waveforms require well-constrained source parameters to avoid mapping source errors into updated Earth models as well as maximizing the number of measurements in the full-waveform inversions (Tape et al., 2009; Maggi et al., 2009; Zhao et al., 2005).

We solve for source solutions using a full-waveform moment tensor methodology computed using synthetic seismograms from both 1-dimensional (1D) and 3-dimensional (3D) tomographic models at two frequency bands. With each inversion we compute a variance reduction from the initial solution, assess the goodness of fit between the data and synthetic seismograms, and determine the stability of each event solution. A quantitative comparison of each inversion case allows for an assessment of the advantages and limitations of different seismological techniques using similar data sets.

\section{Geologic Setting}

Our study region is geographically broad and tectonically diverse, spanning nearly the entire Middle East, parts of western Asia and northern Africa. It can be broken into 7 broad tectonic regions (Figure 1.1): Zagros mountain range, Makran subduction zone, Iranian Plateau, Caspian Basin and surrounding ranges

of the Alborz, Kopet-Dagh, and Talesh mountains, Caucasus, the Arabian Plateau in Saudi Arabia, and Hindu Kush/Tian Shan to the east. The combination of 
convergence between the Arabian and Eurasian plates in the west, and India's collision with Eurasia in the east, has created a complex area of intercontinental mountain belts, deep basins, incipient subduction, and dynamic micro-plates.

Motion of the Eurasian and Arabian plates led to the closure of the Neo-Tethyan Ocean during the late Neogene, with the onset of collision between Arabia and Eurasia occurring between 35 - 23 Ma (Vernant et al., 2004; Hatzfeld and Molnar, 2010; Adams et al., 2009). Intracontinental shortening accommodates most of the convergence, especially in Iran; however, large strike-slip faulting occurs along block margins. The interplay between strike-slip and thrusting motion results in compressional structures that strike obliquely relative to the regional convergence direction (Vernant et al., 2004). The transition zone between strike-slip motion in the Zagros (on the Main Recent Fault and North Anatolian Fault) and the Makran subduction zone is marked by large strike slip motion on the Minab-Zendan-Palami Fault (Vernant et al., 2004). The best estimates for the current motion between Arabia and Eurasia is between 18-25 mm/yr, which is slightly slower than the precollision rate of $31 \mathrm{~mm} / \mathrm{yr}$ (Hatzfeld and Molnar, 2010). Roughly 20\% of ArabiaEurasian convergence is accommodated for in the Zagros (Hatzfeld and Molnar, $2010)$.

To the east, the Hindu-Kush and Tian Shan ranges reflect the transmission of stresses north due to the collision of the Indian subcontinent into Eurasia, beginning roughly during the Tertiary period (55 - $45 \mathrm{Ma}$ ) (Hatzfeld and Molnar, 2010). Similarly to Arabia's collision with Eurasia, the rate of convergence decreased rapidly once plates collided; Hatzfeld and Molnar (2010) cites a precollision rate of $110 \mathrm{~mm} / \mathrm{yr}$ compared with a present rate of 32-44 mm/yr. Reigber et al. (2001), using GPS, quantifies nearly $20 \mathrm{~mm} / \mathrm{yr}$ of deformation accommodated for within the Tian Shan alone. A better understanding of the complex motion between micro and macro plates in the region is needed to unravel the tectonic history and 
structure.

\section{Moment Tensor Inversion Methodology Data Set}

An original earthquake event list, obtained from the Global Centroid-Moment Tensor (Global CMT) Catalog (Dziewonski et al., 1981), was compiled containing events between January 1990 and July 2007; events with a moment magnitude greater than or equal to 5.5 were used. This list contained greater than 200 events within the study region, spanning the Middle East from Turkey to India $\left(30^{\circ} \mathrm{E}\right.$ - $\left.80^{\circ} \mathrm{E}\right)$ east-west and the Horn of Africa to the Kazakh Platform $\left(10^{\circ} \mathrm{N}-50^{\circ} \mathrm{N}\right)$ south-north (Figure 1.1). Broadband seismic waveform data was acquired from IRIS DMC from regional and teleseismic stations; a total of 578 stations from 21 receiver networks were used for the moment tensor (MT) inversions. Typical source-to-station distances ranged from a couple hundred kilometers to $90^{\circ}$.

\section{Inversion}

We follow a moment tensor inversion methodology by Liu et al. (2004), adapted from a local to regional set of earthquakes, and solve for the 6 independent seismic moment tensor elements $\left(M_{i j}\right)$ plus the event depth. We perform inversions for constrained, zero-trace and double-couple, and unconstrained solutions, azimuthally weighted and unweighted, with and without depth variation. The variety of inversion parameterizations was done to assess the stability of each solution, the robustness of the inversion method, and compare the results using different constraints to determine a robust solution. As in Liu et al. (2004), a comparison of the solutions from different parameterizations showed little difference among the solutions driven by the large number of measurement windows and the stability of the method; our focal mechanism solutions remain consistent for each case. A zero-trace, azimuthally-weighted solution while solving for depth, is used for error analysis comparisons between wave speed models and frequencies, as this 
parameterization produced a robust solution with good match between the data and synthetics. Our methodology lends itself to testing the source of non-DC components by comparing solutions between $1 \mathrm{D}$ and $3 \mathrm{D}$ waves speed models to investigate whether a reduction in misfit reflectes imperfections in the model, as suggested by Liu et al. (2004). If the source of the non-DC component is indeed an effect of poorly constrained Earth structure in the model, we should see a reduction in non-DC going from $1 \mathrm{D}$ to $3 \mathrm{D}$ parameterizations.

We define the misfit objective function, E, as in Liu et al. (2004) where,

$$
E\left(m, \mu_{1}, \mu_{2}\right)=\lambda E_{1}(m)+\mu_{1} C_{1}(m)+\mu_{2} C_{2}(m)
$$

$E_{1}$ represents the least-square misfit function,

$$
E_{1}(m)=\frac{1}{2 A_{1}} \sum_{i=1}^{N} w_{i} \int\left[d_{i}(t)-s_{i}(t, m)\right]^{2} d t .
$$

$C_{1}(m)$ is a zero trace moment tensor constraint, $C_{2}(m)$ is a double-couple source mechanism constraint, $\lambda$ is the function weight, $\mu_{1}$ and $\mu_{2}$ are Langrange multipliers associated with the constraints; in equation $1.2, A_{1}$ is a normalization factor, $w_{i}$ represents specified weights (eg. azimuthally weighted, $\left.w_{i}^{a}\right), d_{i}$ and $s_{i}$ are the data and synthetics respectively, and $m$ is the moment tensor. Synthetics are allowed to shift in time to match data.

\section{Synthetic Seismogram Generation}

Full waveform synthetic seismograms are required for the MT inversion. We computed both 1D and 3D synthetics to compare wave speed models independent of the inversion methodology. 1D synthetics were created using mode summation from the PREM (Dziewonski and Anderson, 1981) wave speed model. Synthetics for the 3D reference model case were created using the spectral-element method (SEM) (Komatitsch and Tromp, 1999, 2002a,b) using the S2.9EA (Kustowski et al., 2008) wave speed model. Benefits and details of using the SEM method- 
ology over other methodologies are described in Komatitsch and Tromp (1999, 2002a,b). The computation cost for computation of the 3D synthetic seismograms was significant, but tractable on a dedicated cluster. The S2.9EA model is a global shear-wave velocity structure model based on the PREM reference and determined from surface wave phase velocities, long-period waveforms, and body-wave travel times (Kustowski et al., 2008). The full 3D wave speed model also uses a Crust 2.0 crustal model (Bassin et al., 2000), attenuation from PREM (Dziewonski and Anderson, 1981), and Etopo5 topography/bathymetry (NOAA, 1988). Compressional wave speed perturbations are scaled from shear-wave speed perturbations by 0.55 as in Kustowski et al. (2008). Initial moment tensor solutions were obtained from the Global CMT Catalog (Dziewonski et al., 1981).

Synthetic seismograms and Fréchet derivatives for each component of the moment tensor and depth, were created at all stations within a $90^{\circ}$ by $90^{\circ}$ mesh seen in Figure 1.1, inset. The depth derivative was calculated by the difference between synthetics from the initial solution and synthetics with a depth increased by $d h$. Based on synthetic tests, depth perturbations of 1, 10, 15, 20, 25, and $50 \mathrm{~km}$ show a less than $1 \%$ change in calculated depth derivatives for all perturbations with the exception at $50 \mathrm{~km}$, which is an unreasonable $d h$ value for shallow events. We use a $d h$ value of $1 \mathrm{~km}$.

\section{Data Processing}

Data was filtered between 25-125 second (short period) and 60-125 second (long period) to compare the inversion performance and results at different period bands; a maximum period of 125 seconds was used due to band limitations in instrument response. A bootstrap analysis was performed to assess solution robustness (Press et al., 1997). During the bootstrap analysis, we solved for each event solution 200 times using a random selection of components (radial, vertical, and tangential) from the original dataset. The P-axes were then plotted on a focal sphere to 
quantitatively assess the stability of the plunge and trend, see Figures 1.2.

The Flexwin (Maggi et al., 2009) algorithm was used to automatically select time windows for input into the MT inversion using a combination of criteria based on phase, amplitude, ratio of short term and long term average, and envelope mismatch. Flexwin allows for a large volume of repeatable measurements to be made on full-waveform data-synthetic pairs that would otherwise be over looked when hand picking only peaks for specific phases or amplitudes. Flexwin has usertunable parameters and the ability to adapt to 1D and 3D models (Maggi et al., 2009). As per the user-tunable parameters detailed in Maggi et al. (2009), we required a cross correlation value of 0.75 and an amplitude ratio $(\ln A)$ of $<1.0$ for Flexwin windows to be accepted in the MT inversion. A minimum signal to noise ratio of 3.5 within two measurement windows, and a minimum single window signal to noise ratio of 1.5 was required to use the time series in the MT inversion. Flexwin fine tuning parameters are $c_{0}=0.7, c_{1}=4.0, c_{2}=0.0, c_{3 a, b}=(1.0,2.0)$, $c_{4 a, b}=(3.0,10.0)$, see Maggi et al. (2009) for details. A consistent number of evaluation windows was used for each inversion case, permitting an appropriate comparison without bias to the amount of waveform being evaluated, Table 1.1.

\section{Moment Tensor Inversion Results}

We recovered 184 well constrained solutions out off the initial set of events (for the complete earthquake catalog, see Table 1.2); the remaining 11 events had data quality issues that did not produce acceptable results. The average constraint on the trend and plunge is shown as a histogram in Figures 1.2 and 1.3 and in Table 1.1. Standard errors were determined for the trend and plunge of the MT compressional axes, P-axes, using the bootstrap methodology discussed previously. Standard errors for the trend and plunge of the T-axes were also analyzed; the errors are comparable to the spreads for the P-axes and as such we do not report 
results for the T-axes.

\section{Comparison}

To quantify the effect of wave speed model on the MT inversion, comparisons were made between 1D and 3D moment tensor solutions using an identical data processing scheme and a consistent number of evaluation windows, this allows for direct comparisons of inversion results based on wave speed models and frequency bandwidth without bias to methodology or the number of evaluation windows. The 1D wave speed model does a sufficient job fitting simple body wave signals and large amplitude surface waves at both period bands, example waveforms in Figure 1.4. Complex signals, from body wave propagation and surface wave dispersion due to the continental lithosphere, are not adequately fit by the $1 \mathrm{D}$ model at shorter periods. Employing the 3D wave speed model (Kustowski et al., 2008), synthetic seismograms predict a larger portion of the data at all periods, including the late arriving shorter period arrivals due to strong dispersion from the continental lithosphere. Additionally, using an appropriate 3D model improves the amplitude and phase misfits when compared to a 1D model and facilitates the use of more waveform data in the MT inversion.

A quantitative comparison of the variance reduction between 1D and 3D models shows that, within the same frequency band, a reduction in error on the trend and plunge is seen when using the 3D versus the 1D model, Table 1.1. At longer periods, $60-125 \mathrm{~s}$, the difference between using the 1D and 3D model is negligable, seen only as an approximate difference of $0.39^{\circ}$ on the trend and plunge. At shorter periods, 25-125s, the improvement is more significant, reducing the error by approximately $4.43^{\circ}$ on the trend and $1.34^{\circ}$ on the plunge, see Figure 1.3.

A metric was created to ease the comparison between inversion runs by defining a variable $\tau$ as

$$
\tau=\lambda_{1} \overline{|1-C C|}+\lambda_{2} \overline{|\Delta \ln A|}+\lambda_{3} \overline{|\xi|}-\lambda_{4} \frac{N}{200}+\lambda_{5} \frac{A Z}{360}
$$


where $\lambda_{1-5}$ represents the relative weights $\left(\lambda_{1}=5, \lambda_{2}=0.25, \lambda_{3}=1, \lambda_{4}=\right.$ $\left.0.05, \lambda_{5}=0.05\right), \overline{C C}$ is the average cross-correlation value, $\Delta \overline{\ln } A$ is the average amplitude ratio, $\bar{\xi}$ is the average misfit, $N$ is the number of windows used, and $A Z$ is the maximum azimuthal gap. Weights were chosen to emphasize the importance of the cross-correlation, $\overline{C C}$, and misfit, $\bar{\xi}$, to the goodness-of-fit, and downweight the larger numbers associated with the $N$ and $A Z$ variables. Based on this defined metric, where a smaller $\tau$ value indicates better goodnes-of-fit, $95 \%$ of the events have $\tau$ values $<10$ and $85-90 \%$ have $\tau$ ranging between 0 and 2 . We calculate the mean $\tau$ and standard deviation for each inversion set (1D25, 1D60, 3D25, 3D60) to obtain a single number with which to evaluate the entire dataset. Events with metric values, $\tau>10$ are considered to be very poorly fit and are not included in the inversion set averages. Typically, an event with a large metric, or poor goodness-of-fit has severe data quality or lack of data issues resulting in values of $\tau$ in the $>>100$

Based on trend and plunge standard error improvements, increases in the crosscorrelation coefficient and variance reduction for short periods (25-125s), (Table 1.1 and Figures 1.3 and 1.4) the 3D S2.9EA model (Kustowski et al., 2008) produces a better fit to the data than does the 1D PREM model (Dziewonski and Anderson, 1981). At shorter periods waveform fit deteriorates slightly, relative to the longer period data (60s vs. 25s), as seen by the decrease in cross-correlation value and increase in the mean $\tau$ (Table 1.1); however the benefit is the incorporation of much more seismic data into the source inversion as a result of 3D synthetics predicting a larger portion of the waveform data (Fig. 1.4) .

Results compare favorably with previously published solutions in both mechanism and depth (Jackson et al., 2002; Tatar et al., 2004; Talebian and Jackson, 2004), as well as Global CMT solutions. Figure 1.5 shows a comparison between Global CMT solutions for 3 events and our calculated solutions, for each event our 
calculated source mechanism are similar to Global CMT.

\section{Depth}

Comparison of our revised depth versus Global CMT depths are made in Figure 1.6. Differences between $1 \mathrm{D}$ and $3 \mathrm{D}$ wave speed models are subtle, yet present, especially for shallow events. Agreement between our determined depths and those from Global CMT improves with the inclusion of shorter period data (25-125s), this is especially true at shallow depths. Lack of Global CMT event depths less than $15 \mathrm{~km}$ is a result of a constraint imposed on their solution, our inversions do not impose this constraint. A cluster of events between $50-150 \mathrm{~km}$ depth is within agreement with that of Global CMT with the inclusion of shorter periods. For deep events $(>200 \mathrm{~km})$ there is a systematic divergence between our solutions and those from Global CMT as the calculated depth is shallower than the initial depth; this is most likely due to S2.9EA's heterogeneous wave speeds at depth. Additionally, Muyzert and Snieder (1996) has shown that these large deviations we see in the depth may possibly be due to unstable initial phase behavior in the long period surface waves.

\section{Discussion}

The comparison between Global CMT solutions and the moment tensor solutions presented here show minimal difference in source mechanism, an average of $7.31^{\circ}$ and $7.56^{\circ}$ difference for the $\mathrm{P}$ and $\mathrm{T}$ axes respectively for the $3 \mathrm{D} 25-125 \mathrm{~s}$ case. This is true for all four cases examined (1D vs 3D model, minimum period $25 \mathrm{~s}$ vs $60 \mathrm{~s})$ and when varying constraints applied to the inversion. In all cases, a reduction in variance between data and synthetic is seen between the original solution and our calculated solutions. The greatest reduction in variance is seen in the 3D case when filtered between 25-125s, shown in Table 1.1. A majority of events see a variance reduction of $5-40 \%$ which represents a significant improvement in fitting waveforms. Events with the largest variance reduction are a result 
of a better amplitude agreement between the data and synthetic.

The largest contribution to the misfit in our result can be attributed to poorly constrained shallow Earth structure in our models. The use of shorter periods (25-125s) introduces a potential for contamination from larger errors due to unresolved wave speed structures. At shorter periods, Earth structure will have a greater influence on the propagating wavefield and errors in the Earth model may be mapped into the source solution. There is a small, but significant, increase in standard error on the trend and plunge as well as a decrease in the average cross-correlation value from $60-125$ s to $25-125$ s (Table 1.1 ). We do not feel the degradation in solution stability, as seen by the increase in trend and plunge standard error, and waveform metrics, as seen by the decrease in the cross-correlation value, are justification for the removal of shorter period signal. Alternatively, the addition of shorter period signals, $25-125 \mathrm{~s}$, allows for much more seismic data to be incorporated into the inversion, as seen by an increase in the number of evaluation windows from 1D25 to 3D25 (Table 1.1), indicating a robust predictive capability of the 3D wave speed model over a wide period range. An azimuthal gap in seismic stations seen in Kazakhstan and Russia also contributes to the error of the trend of some solutions, but for most events the overall azimuthal coverage is excellent and the trend is well constrained for the entire data set as a whole when using the 3D moment tensor inversion.

The percentage of double-couple, defined by decomposing the moment tensor into a double-couple and CLVD component (Stein and Wysession, 2009), increases when using a 3D model relative to the $1 \mathrm{D}$ model results, implying the non-doublecouple component of the solution comes from poorly constrained wave speed structure in the $1 \mathrm{D}$ model relative to $3 \mathrm{D}$. The nature of observed slip on faults being both non-planar, as in rotational faulting, and/or events with complex rupture histories, may manifest itself as volumetric change (i.e. non-double-couple) within 
the source (Julian et al., 1998). We plot the percentage of double-couple components in our solutions using a 1D and 3D model at 25-125s periods, Figure 1.7, to test this hypothesis that non-double-couple components are a reflection of poorly constrained regional structure in the initial velocity model or complex fault geometry, consistent with suggestions made by Liu et al. (2004) and Henry et al. (2002). The number of events in our catalog with significant non-double couple components suggests that improvements in the wave speed model are needed. Experiments by Hjörleifsdóttir and Ekström (2010) to evaluate source parameters using synthetic seismograms at periods greater than $40 \mathrm{sec}$, show small errors in the non-doublecouple component when using a diverse seismic data set, similar to the global catalogs (Miller et al., 1998). Experiments here using real data and are consistent with synthetic experiments at longer periods, but the use of a 3D wave speed model and shorter periods, $25 \mathrm{sec}$, significantly increase the double-couple component. This may suggest a frequency dependance to resolving the non-double-couple components. At longer periods (60-125s), not shown, the difference between 1D and 3D percentage double-couple is minimal, further suggesting that 3D structure at short periods is the cause for increased double-couple component. We would expect an increase in the percentage of double-couple components in the source with improvements made to the wave speed model; however due to the complex nature of real faulting, a completely double-couple source is unlikely. A subset of our deepest events $(100$ to $\sim 260 \mathrm{~km})$ shows a similar trend as for the whole catalog.

\section{Tectonics}

Comparisons between our solutions with regional tectonic features show good general agreement with previous geologic interpretations (Figure 1.8). Within the Zagros, right-lateral strike slip and northeast striking convergence is dominant, corresponding to right-lateral strike slip motion on the Main Recent Fault and general 
northeast motion reflecting the collision of the Arabian plate with Eurasia and consistent with tectonic interpretations made by Talebian and Jackson (2004), Tatar et al. (2004) and Hatzfeld and Molnar (2010). Northeast striking thrust events also dominate in the Caspian Basin region and Alborz mountain range as also seen in Jackson et al. (2002). Two large strike-slip mechanisms, one in the Kopet-Dagh region and another near the border of Iran and Afghanistan reflect rotation around rigid blocks within central Iran (Vernant et al., 2004; Hollingsworth et al., 2006, 2008, 2009; Hatzfeld and Molnar, 2010). In Turkey large strike-slip mechanisms are consistent with motion along the North Anatolian Fault and East Anatolian Fault to the east. Within the Red Sea, tensile mechanisms reflect extension within the basin. Other notable trends are seen in the Gulf of Aden, where there is a cluster of non-double couple strike slip events. The Hindu Kush/Tian Shan regions are dominated by reverse and normal faults, the strikes vary considerably reflecting the complexity of the regional structure and collision of the Indian subcontinent and Eurasia.

\section{Conclusions}

Using a full-waveform moment tensor inversion method (Liu et al., 2004), we repeat an identical data processing scheme for four cases using two initial models (1D and 3D) and two frequency bands, allowing for direct comparison between results and the evaluation of model and frequency bandwidth. The number of evaluation windows is consistent for each inversion set permitting an appropriate comparison between models and frequency ranges without bias given to the number of evaluations.

We provide justification for the use of $3 \mathrm{D}$ models, in preference to $1 \mathrm{D}$ models, by showing a reduction in variance and better constraint on moment tensor solutions, source characteristics, Earth structure and event depth. This is espe- 
cially true in regions of highly heterogeneous Earth structure, as seen in our study region. The $1 \mathrm{D}$ model does not provide an adequate fit to waveforms at shorter periods, especially in regards to fitting complex body wave propagation and surface wave dispersion. Additionally, the $3 \mathrm{D}$ model produces a solution with a greater percentage of the source approximated as a double couple, suggesting that the non-double-couple component of our solutions comes from poorly constrained wave speed structure.We achieved an overall agreement in mechanism and depth with regional tectonics across inversion methods, wave speed models, and frequency range confirming the stability and robustness of our methodology and solutions. Further, the solutions obtained in this study agree with those found by pervious researchers, Global CMT, and also agree with the large scale geologic structures and overall GPS measurements (Adams et al., 2009; Jackson et al., 2002; DeMets et al., 1994; Vernant et al., 2004; Tatar et al., 2004; Hatzfeld and Molnar, 2010).

\section{Data and Resources}

The facilities of the IRIS Data Management System, and specifically the IRIS Data Management Center, were used for access to waveform and metadata required in this study. The IRIS DMC is funded through the National Science Foundation and specifically the GEO Directorate through the Instrumentation and Facilities Program of the National Science Foundation under Cooperative Agreement EAR0552316. The Global Centroid-Moment Tensor (Global CMT) Catalog was used to compile earthquake events by using http://www.globalcmt.org/CMTsearch. html (last accessed May 2012). Synthetic seismogram simulations were carried out on supercomputing facilities at the University of Rhode Island and Princeton University. Map figures were produced using GMT (Wessel and Smith, 1991). 


\section{Acknowledgments}

We would like to thank Qinya Liu for providing the codes to calculate our source mechanism solutions and for her helpful comments throughout the process; Daniel Peter for his help creating synthetic seismograms; Arthur J. Rodgers, Jeroen Tromp, and Yang Shen for providing valuable insight. We would also like to thank three anonymous reviewers for their helpful comments and suggestions. This research was supported by the Air Force Research Laboratory under contract No. FA8718-08-C-0009. 


\begin{tabular}{l|c|c|c|c}
\hline & $1 \mathrm{D} 60(\mathrm{~s})$ & $1 \mathrm{D} 25(\mathrm{~s})$ & $3 \mathrm{D} 60(\mathrm{~s})$ & $3 \mathrm{D} 25(\mathrm{~s})$ \\
\hline Trend $\left(^{\circ}\right)$ & $4.71 \pm 8.08$ & $9.66 \pm 15.95$ & $5.10 \pm 9.37$ & $5.23 \pm 9.18$ \\
Plunge $\left(^{\circ}\right)$ & $3.22 \pm 4.69$ & $4.33 \pm 8.69$ & $2.83 \pm 4.37$ & $2.99 \pm 5.65$ \\
Avg. Cross Correlation & 0.92 & 0.82 & 0.93 & 0.87 \\
Avg. dlnA & 0.16 & 0.41 & 0.16 & 0.37 \\
Avg. \# Windows & 387.07 & 338.53 & 267.71 & 363.99 \\
Mean $\tau$ & $1.07 \pm 1.26$ & $1.96 \pm 1.22$ & $1.11 \pm 1.59$ & $1.52 \pm 1.25$ \\
Variance Reduction & 20.12 & 24.17 & 33.42 & 36.77 \\
Mean \% & \multicolumn{2}{|c}{} \\
\hline
\end{tabular}

Table 1.1: Comparison between 1D and 3D inversion sets. Standard error on the trend and plunge of the $\mathrm{P}$-axes for all events is calculated from the results of a bootstrap analysis; standard error of the T-axes show a comparable spread and is not reported. Also shown, is the result of calculating the average and standard deviation of $\tau$ from equation 1.3 for each inversion set. A full dataset, mean reduction in variance between the data and misfit between the initial solution, $M_{0}$, solution and our calculated solution is shown. 


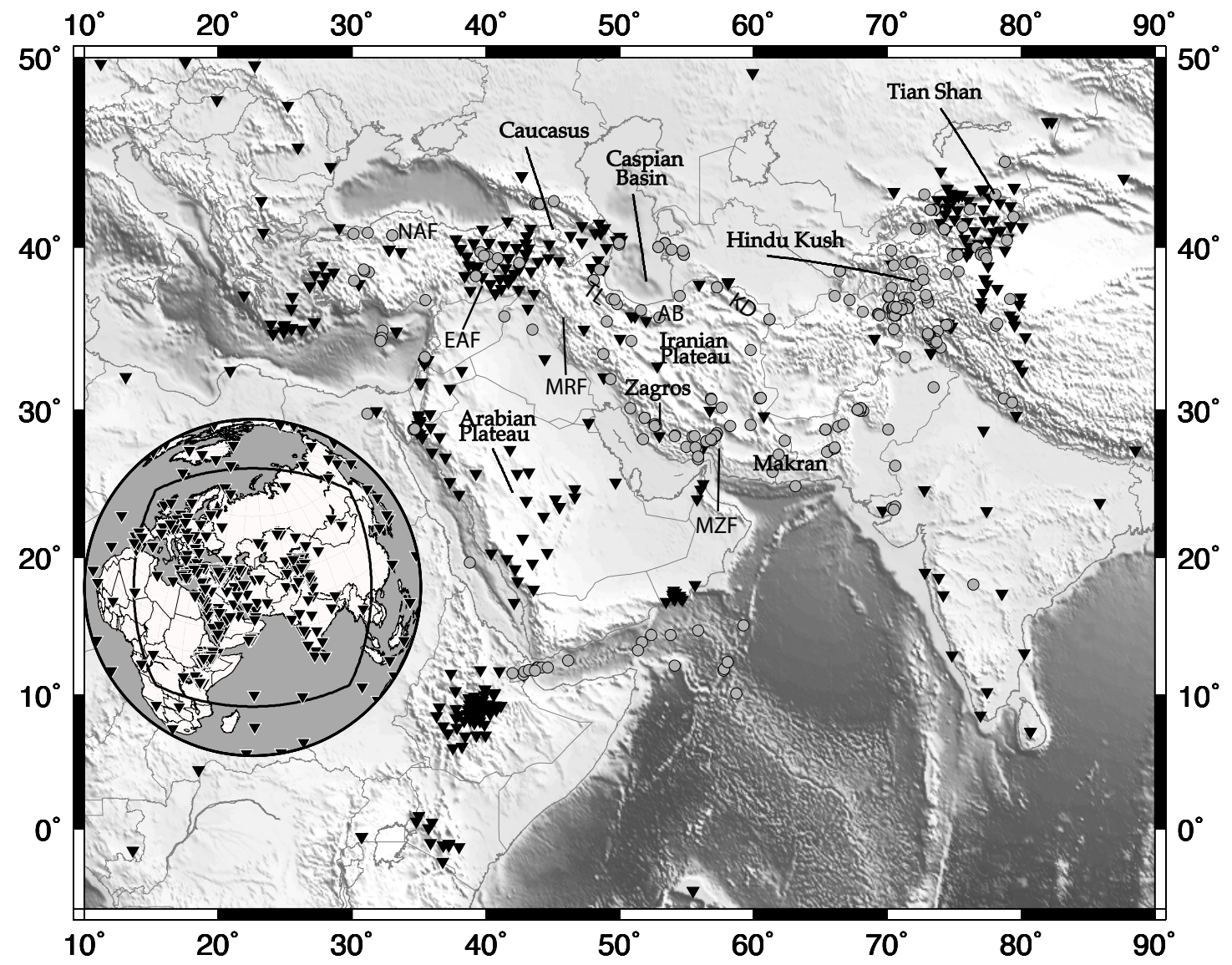

Figure 1.1: Events (circles) and stations (triangles) used for this study within the Middle East. Inset shows the global distribution of 580 stations used with the 3D S2.9EA wave speed model. Synthetic seismograms were created using the specfem3d software package and calculated for all stations located within the $90^{\circ}$ by $90^{\circ}$ mesh (black box). Major faults and mountain ranges discussed in the paper have been labeled: North Anatolian Fault (NAF); East Anatolian Fault (EAF); Main Recent Fault (MRF); Minab-Zendan-Palami Fault (MZF); Talesh (TL), Alborz (AB), and Kopet-Dagh (KD) Mountain Ranges. 

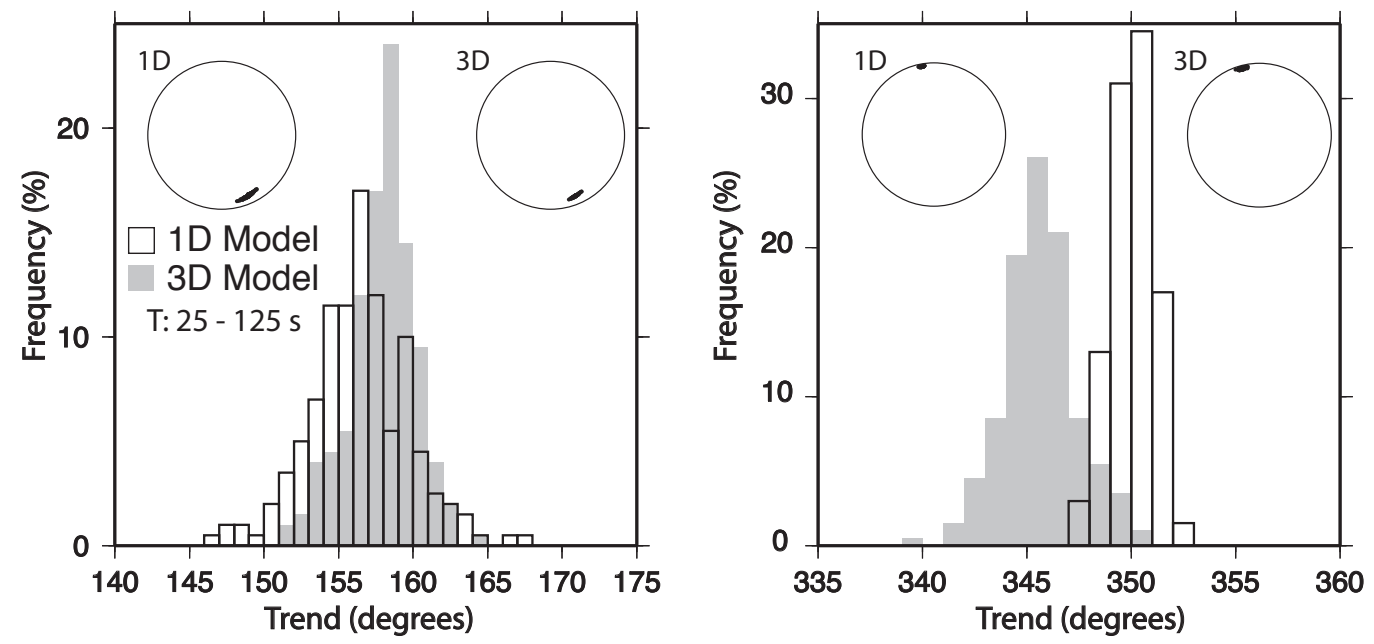

Figure 1.2: Results of a bootstrap statistical analysis for two single events, a)1997/05/13 and b)2005/02/22. Plotted in the upper left and right are the P-axes on a focal sphere after each inversion $(n=200)$ using a $1 \mathrm{D}$ and 3D model. A tighter cluster of dots indicates a more constrained solution and a smaller standard error on the P-axes of the solution. The histogram shows the distribution of results for the trend $(n=200)$ for each particular event. 
(a)

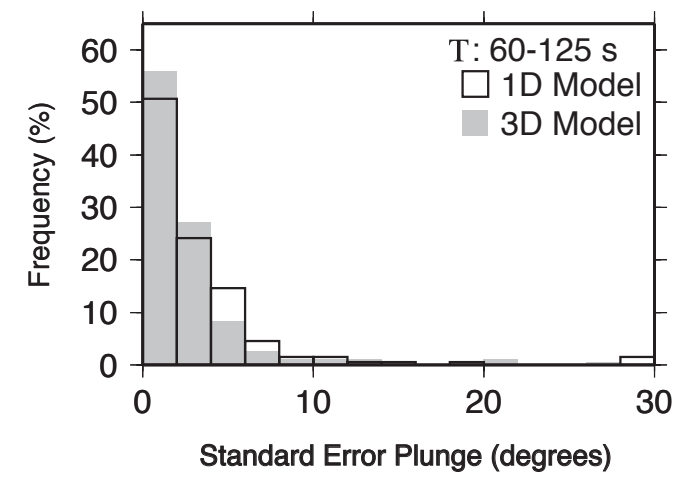

(c)

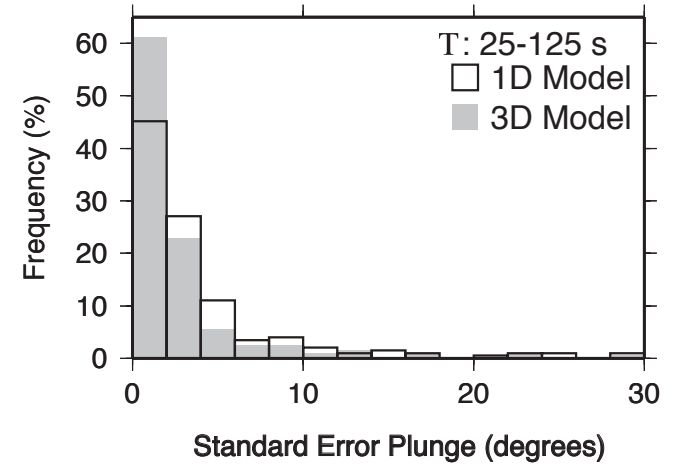

(b)

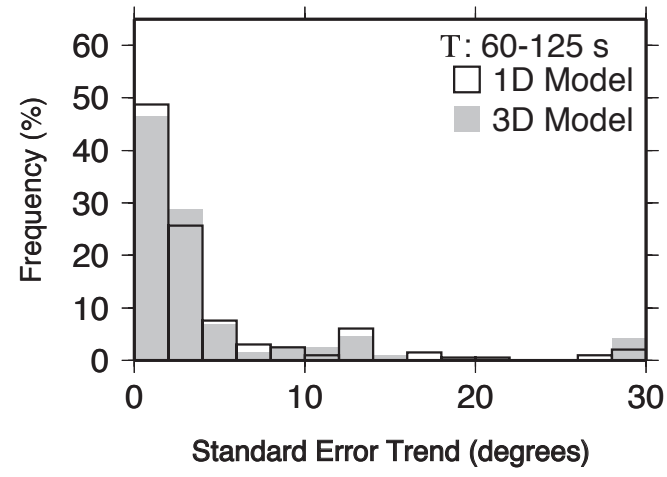

(d)

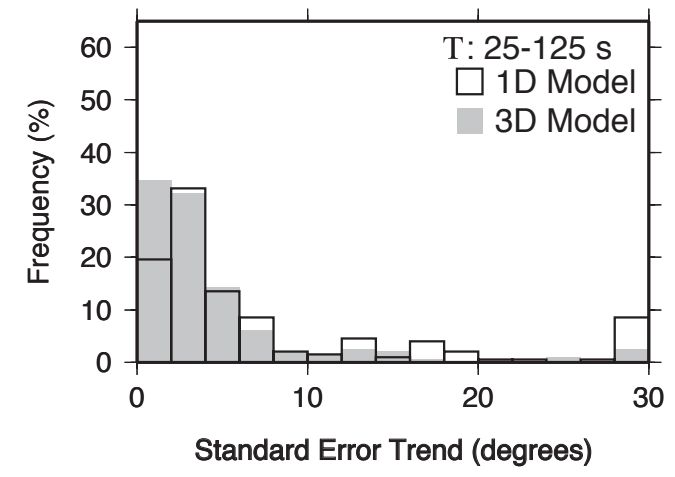

Figure 1.3: Moment tensor inversion standard error for all events, $n=195$. Histograms show the standard error for two frequency bands, 60-125s (a) and (b) and 25-125s (c) and (d), on the trend and plunge. The last bin represents standard errors $>30^{\circ}$. Grey bars represent the use of 3D Kustowski et al. (2008) derived synthetics. White bars represent using 1D model Dziewonski and Anderson (1981) derived synthetics. 


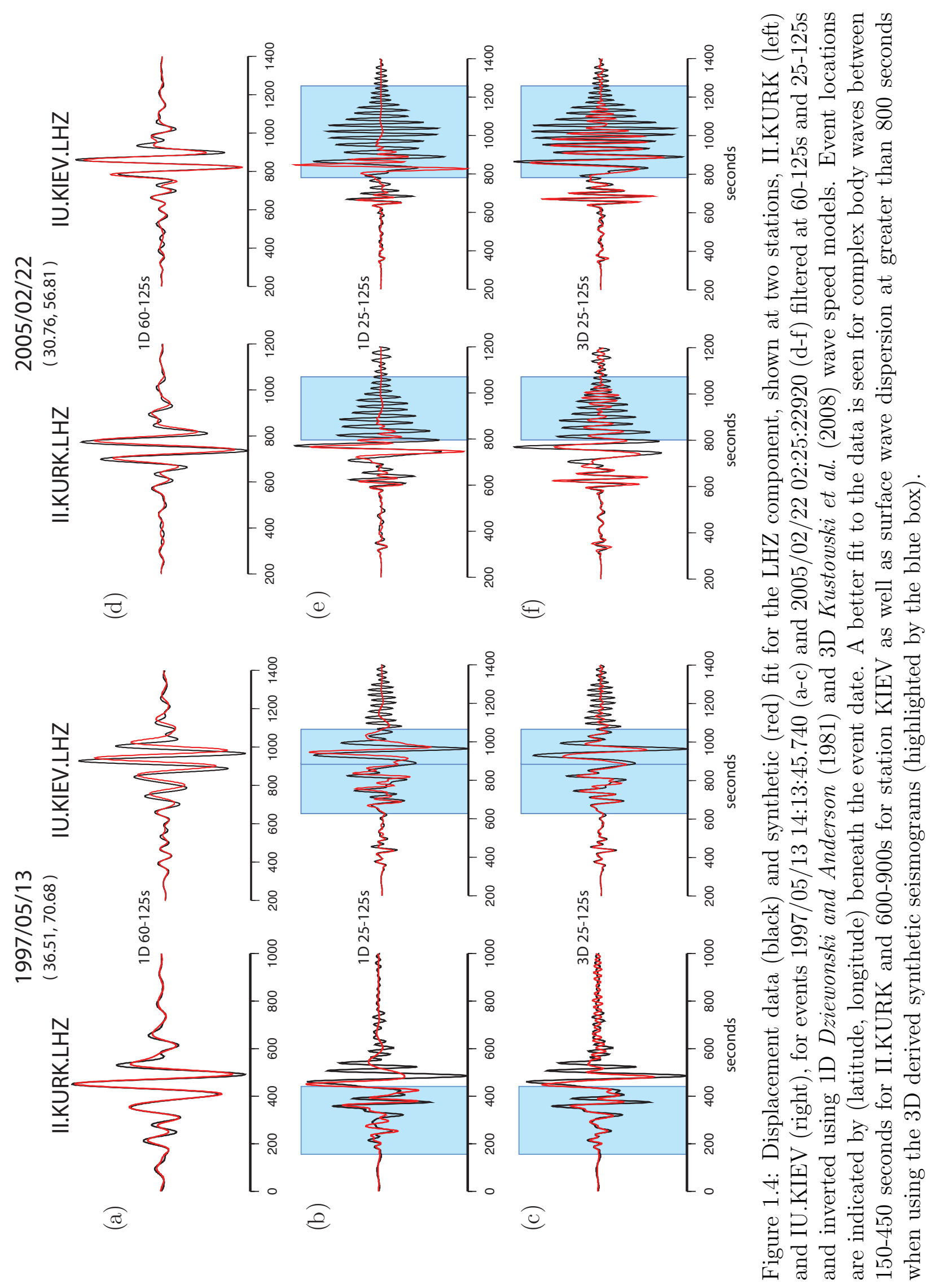




\section{Initial Solution}

\section{D Zero Trace}

(a)

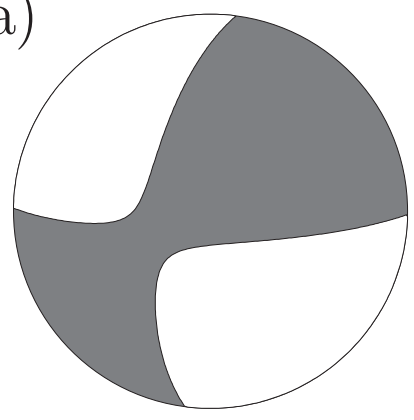

(c)

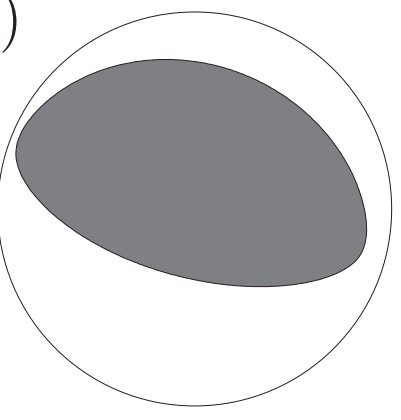

(e)

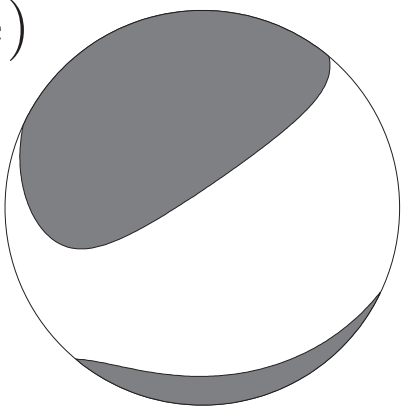

2007/01/08

17:21:49.910

$22.36 \%$

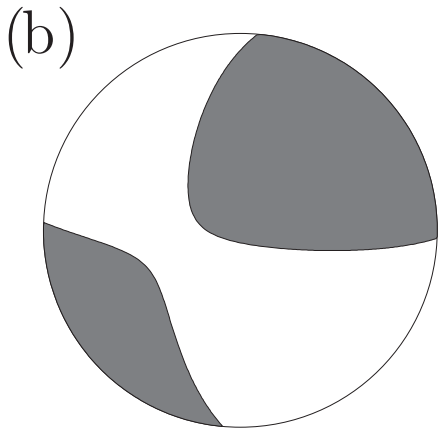

$2002 / 06 / 22$

02:58:23.010

$19.66 \%$

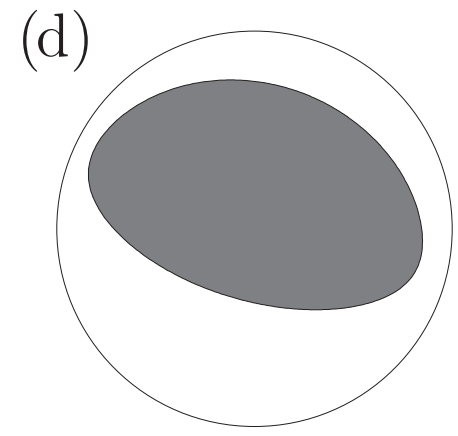

2000/05/12

23:10:29.980

$15.10 \%$

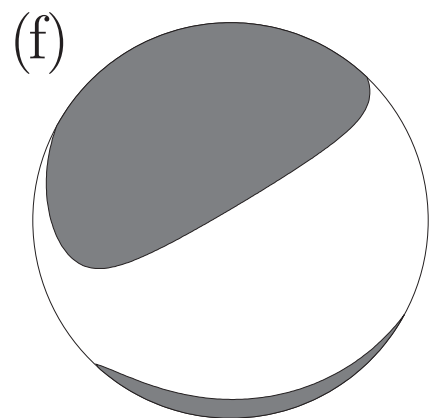

Figure 1.5: Comparison between Global CMT solution (left) and 3D azimuthally weighted 7-parameter solution (right). Our solutions compare well with the initial solution, but variance between the data and synthetic is reduced while fitting more of the data using a 3D model. Percentage represents the variance reduction between our solutions and the Global CMT solution. A majority of events in the catalog (Table S1) see a variance reduction between 5-40\% compared to the Global CMT solution. 
(a)

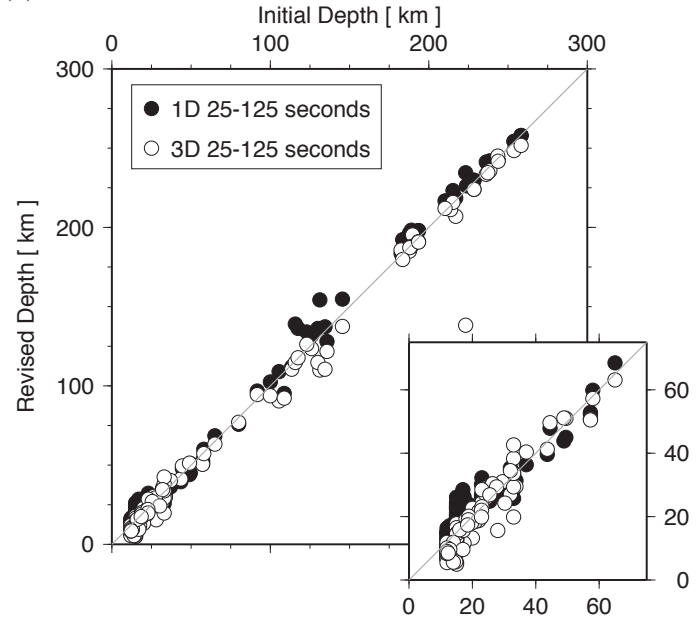

(b)

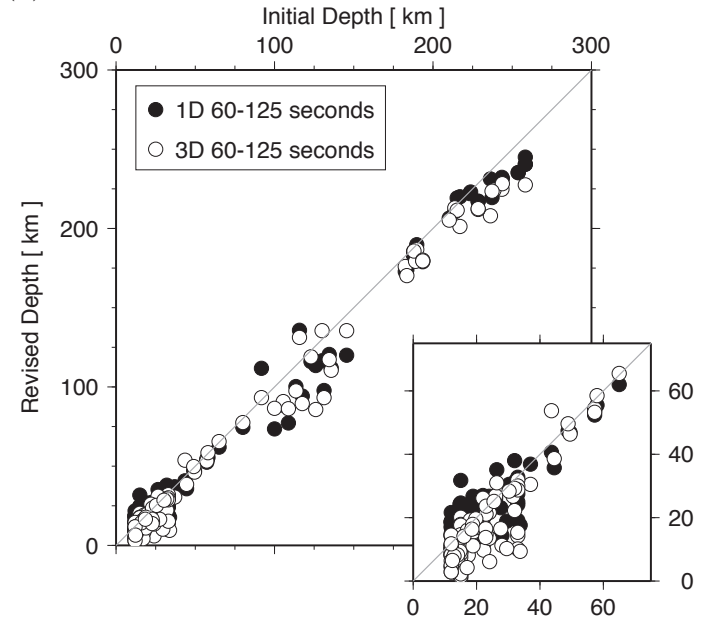

Figure 1.6: Original Global CMT depth (Initial Depth) plotted against this study's depths (Revised Depth) from the zero-trace azimuthally weighted solution; a line represents a 1-1 relationship between the two depth solutions. (a) Shows short period (25-125s) solutions which have a tighter fit to the 1-1 line than do (b) longer period (60-125s) solutions. Inset zoomed in to show results at shallow depths. Depth residuals are smaller than symbol size (average residual is less than $2.5 \mathrm{~km}$ ) and therefore not plotted. 


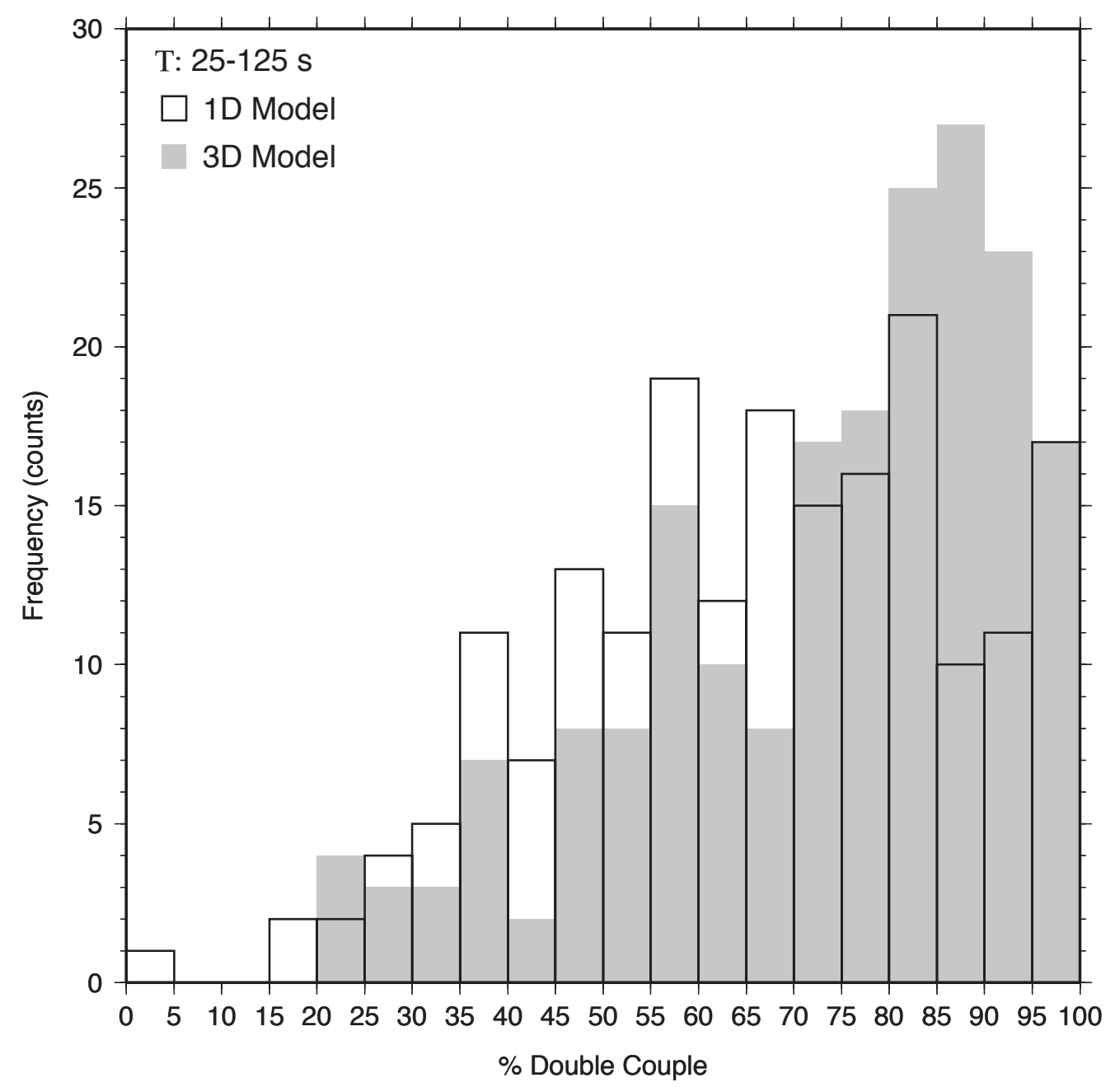

Figure 1.7: Histogram showing the percentage double couple (DC) component for short period (25-100 seconds) solutions. A larger \%DC is seen when using 3D derived synthetic seismograms (grey) compared to 1D derived synthetics (white). This result provides evidence that the $\%$ of non-DC component in the solutions is a result of imperfect and poorly resolved Earth structure within the initial velocity models. 
(a)

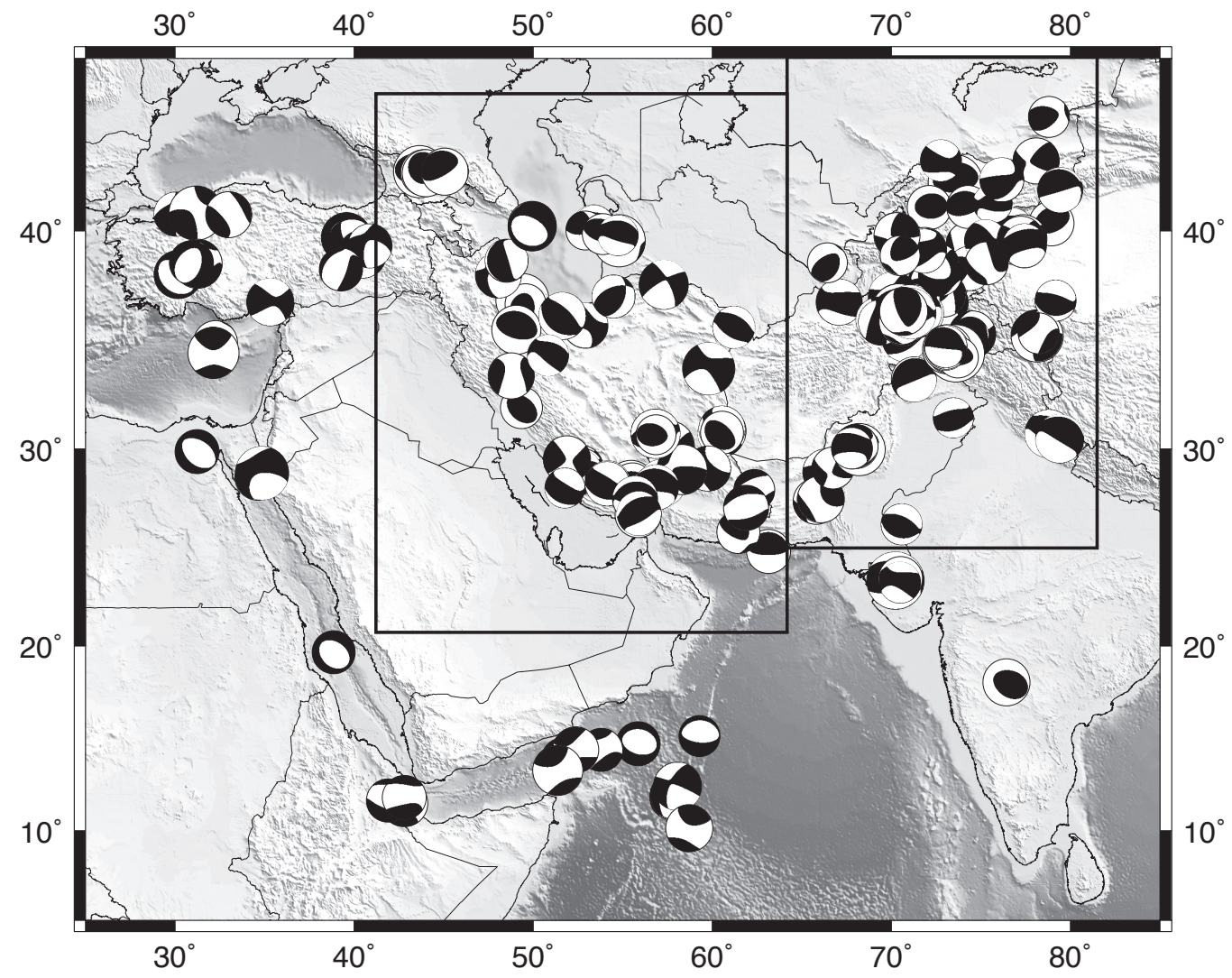

(b)

(c)
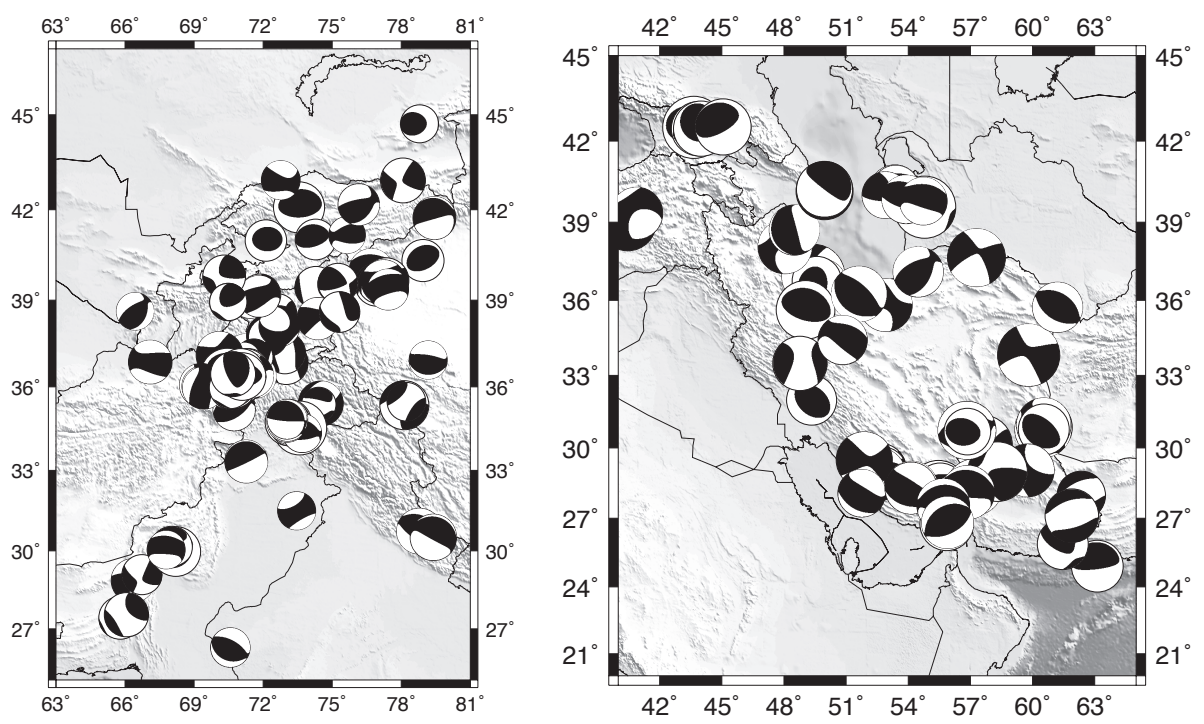

Figure 1.8: Earthquake event solutions for the (a) entire dataset and highlighted regions, (b) Hindu Kush and Tian Shan and (c) Iran. Shown are 195 solutions from the 3D zero-trace azimuthally weighted moment tensor inversion. Solutions agree well with regional tectonics and large scale plate motions. 


\section{Appendix}

Supplemental (1.2): Earthquake catalog containing 184 event solutions solved for using a 3D moment tensor inversion method (Liu et al., 2004). Results are azimuthally weighted, 7-parameter (Mij and depth), zero-trace solutions. Mij components are normalized by dividing by $1 x 10^{17} \mathrm{~N} . \mathrm{m}$. 


\begin{tabular}{|c|c|c|c|c|c|c|c|c|c|c|c|c|c|c|c|c|}
\hline \multicolumn{3}{|c|}{ Date } & \multicolumn{3}{|c|}{ Time } & Latitude & Longitude & Depth & Mw & Mrr & Mrt & Mrp & Mtp & Mtt & Mpp & VarRed \\
\hline 19900 & 01 & 20 & 01 & 27 & 11.00 & 35.5800 & 53.2900 & 29.6094 & 5.80717 & 1.45320 & 2.66950 & -0.14810 & 5.49140 & -2.44250 & 0.98930 & 24.7369 \\
\hline 1990 & 02 & 05 & 05 & 16 & 45.10 & 36.5600 & 70.8400 & 110.6157 & 6.18708 & 17.95600 & -1.79770 & -7.36690 & 0.37620 & -25.63980 & 7.68380 & 37.4747 \\
\hline 1990 & 03 & 04 & 19 & 46 & 22.10 & 28.6600 & 66.1600 & 15.6129 & 5.99898 & 1.13200 & 2.55000 & 3.22550 & -10.65150 & -5.67060 & 4.53850 & 3137 \\
\hline 1990 & 03 & 05 & 20 & 47 & 3.50 & 37.0400 & 72.8500 & 15.7348 & 5.97139 & -7.38520 & 4.13430 & -3.79410 & -2.70050 & -3.40560 & 10.79070 & .7533 \\
\hline 1990 & 04 & 17 & 01 & 59 & 28.40 & 39.2900 & 74.7800 & 15.5878 & 5.81621 & -0.35560 & 0.44760 & -3.92140 & -1.81410 & -4.87840 & 5.23390 & 81.7252 \\
\hline 1990 & 05 & 17 & 13 & 21 & 7.30 & 38.1200 & 74.5800 & 114.7634 & 5.68956 & 0.39070 & 1.06360 & 1.93140 & -1.89070 & -3.35970 & 2.96900 & 6.6017 \\
\hline 1990 & 06 & 17 & 04 & 51 & 46.10 & 26.7500 & 65.2500 & 11.7372 & 5.97755 & 4.29240 & 2.84640 & -6.15020 & -7.49390 & -6.59830 & 2.30590 & 53.6939 \\
\hline 1990 & 06 & 20 & 21 & 00 & 8.50 & 36.9500 & 49.5200 & 14.3413 & 6.92754 & 131.86600 & -233.19400 & -92.64690 & 117.52800 & 13.17670 & -145.04300 & 97.7853 \\
\hline 1990 & 06 & 21 & 09 & 02 & 13.70 & 36.5100 & 49.7700 & 14.6536 & 5.40261 & 1.15230 & 0.03640 & 1.05040 & 0.03160 & 0.09910 & -1.25140 & 96.1907 \\
\hline 1990 & 07 & 13 & 14 & 20 & 43.70 & 36.6800 & 70.6100 & 207.0632 & 6.38577 & 29.68100 & 35.71320 & -5.45020 & 8.72840 & -30.16010 & 0.47910 & 26.8210 \\
\hline 1990 & 09 & 08 & 19 & 33 & 19.60 & 27.5500 & 66.2300 & 13.5112 & 5.47420 & 1.93850 & 0.25360 & -0.20280 & -0.72980 & -1.82790 & -0.11060 & 42.1186 \\
\hline 1990 & 09 & 12 & 15 & 28 & 35.60 & 14.5300 & 59.1100 & 14.6659 & 5.36323 & -1.08140 & -0.88980 & 0.13750 & -0.01580 & 1.05100 & 0.03040 & \\
\hline 1990 & 10 & 25 & 04 & 53 & 46.50 & 35.1900 & 7400 & 121.7054 & 5.86025 & 6.89210 & 0.05150 & 740 & -3.58670 & -6.54880 & -0.34330 & 31.2622 \\
\hline 1990 & 11 & 03 & 16 & 39 & 55.60 & 39.1500 & 71.0100 & 15.6963 & 5.24590 & 0.82190 & & 0.10360 & -0.46010 & -0.60420 & -0.21770 & \\
\hline 1990 & 11 & 06 & 18 & 45 & 54.10 & 28.0600 & 2500 & & 6.50520 & 63.88360 & 24.16860 & 19.62080 & 6.71360 & -65.40940 & 1.52580 & 10.0871 \\
\hline 1990 & 11 & 12 & 12 & 28 & 49.00 & 43.1800 & 78.2400 & 14.5299 & 6.05844 & 3.07250 & 1.68090 & -6.01240 & -6.65750 & 5760 & 10.58510 & 84.2291 \\
\hline 1991 & 01 & 31 & 23 & 03 & 34.90 & 36.0100 & 70.2300 & 123.6140 & 6.56079 & 68.22790 & 54.62590 & -26.68180 & 17.63970 & -22.76310 & -45.46490 & 66.8506 \\
\hline 1991 & 02 & 25 & 14 & 30 & 29.30 & 40.3400 & 79.2000 & 9.9678 & 5.84987 & 7.08270 & 880 & 590 & -2.54710 & -5.08450 & -1.99820 & 65.6194 \\
\hline 1991 & 04 & 29 & 09 & 12 & 47.20 & 42.6000 & 43.6100 & 20.6384 & 469 & 8800 & 51. & 9400 & 14. & 8600 & -16.40280 & \\
\hline 1991 & 04 & 29 & 18 & 30 & 40.70 & 42.3800 & 500 & 069 & 277 & 10.65470 & & 440 & 9310 & -9.06620 & -1.58850 & 41.4633 \\
\hline 1991 & 05 & 22 & 16 & 29 & 2.20 & 27.0400 & 300 & 13.7 & 5.2 & 0.8 & & & 0.4 & -0.7 & -0. & \\
\hline 1991 & 06 & 15 & 00 & 59 & 20.30 & 42.5800 & 700 & 8.3 & 6.1 & 070 & 440 & & 3.2 & -4.30830 & 0240 & 0564 \\
\hline 1991 & 10 & 19 & 21 & 23 & 15.50 & 30.2200 & 2400 & 17.8 & 219 & 1890 & 770 & -9.8 & 15.01220 & -22.12370 & 520 & 867 \\
\hline 1991 & 11 & 08 & 15 & 13 & 43.80 & 26.4700 & 7000 & & 595 & 310 & 340 & 440 & 9260 & -0.98870 & 4540 & \\
\hline 1991 & 11 & 28 & 17 & 19 & 54.80 & 36.8 & 49.3300 & 14.8606 & 5.36354 & 1.25190 & -0.48650 & 0.55020 & -0.3 & 8410 & -0.96780 & 85.7565 \\
\hline 1992 & 01 & 30 & 05 & 22 & 1.40 & 24. & 00 & 16.3 & 359 & 1.93860 & & 0.0 & 0.0 & 7770 & -0.23090 & 30. \\
\hline 1992 & 02 & 26 & 03 & 45 & 19.70 & 11. & & & & & & -4.6 & 570 & -6 & & \\
\hline 1992 & 03 & 05 & 08 & 55 & 6.50 & & & & & -5.74260 & -7. & 2.3 & 1960 & 60 & -14. & \\
\hline 1992 & 03 & 13 & 17 & 18 & 40.10 & 39.9 & & & & -69.30660 & 22. & -18.7 & 370 & 300 & 51. & \\
\hline 1992 & 04 & 24 & 07 & 07 & 25.10 & 27.4 & & & & -1.42210 & & 860 & 7720 & -6. & 5550 & \\
\hline 1992 & 05 & 10 & 04 & 04 & 32.8 & 37.2 & & & & -3.9 & & 50 & 430 & 20 & & 74 \\
\hline 1992 & 05 & 19 & 12 & 24 & & & & & & & & & & & -0 . & \\
\hline 1992 & 05 & 20 & 12 & 20 & 35.00 & 32.9 & & & & 1.16830 & 6.2 & & & -1.2 & & \\
\hline 1992 & 08 & 19 & 02 & 04 & 36.5 & 42.1 & 0 & & 022 & 431.42500 & 231. & -10. & 7060 & -377 . & 190 & \\
\hline 1992 & 08 & 28 & 00 & 50 & 54.20 & 28.4300 & & & & & -0. & -1. & -1.330 & -1.9 & & 8.7390 \\
\hline 1992 & 10 & 12 & 13 & 09 & 56.30 & & & & & & & & -2.329 & & & \\
\hline 1992 & 10 & 23 & 23 & 19 & 47.20 & & & & & & & 11. & & & 60 & 72. \\
\hline 1992 & 11 & 12 & 20 & 41 & 4.70 & & & 184.9 & & & & & & & 80 & \\
\hline 1992 & 11 & 23 & 23 & 11 & 9.10 & & & & & & & & & & 510 & \\
\hline 1992 & 12 & 04 & 11 & 36 & 36.30 & 37.3500 & & 110.1 & & & & & & & & \\
\hline 1992 & 12 & 17 & 10 & 39 & 28.80 & & & & & & & & & -0.2 & -1.77440 & \\
\hline 1993 & 03 & 13 & 17 & 12 & 26.30 & 19.4200 & 500 & 5.0149 & 7649 & -3.1 & & 930 & -1.05290 & 1.60030 & 1.41280 & 52.4978 \\
\hline 1993 & 03 & 16 & 22 & 59 & 45.40 & 11.4900 & & & & & & & & & 0.19120 & \\
\hline 1993 & 08 & 03 & 12 & 43 & 4.50 & 28.6200 & 1000 & & 468 & -9.7 & & -5.62450 & -4.01130 & -1.71310 & 11.50530 & 45.1078 \\
\hline 1993 & 08 & 03 & 16 & 33 & 20.60 & & & & & 560 & & -2.38790 & & -0.58270 & 1.37830 & \\
\hline 1993 & 08 & 09 & 12 & 42 & 49.70 & & & 211.1562 & & 149.92800 & 109.08500 & -65.92220 & -59.70100 & -156.35500 & & 44.5476 \\
\hline 19930 & 09 & 04 & 11 & 38 & 39.40 & 36.1600 & 70.5100 & 195.3544 & 6.00066 & 9.01220 & 6.33000 & 5.79260 & -1.02660 & -9.36660 & 0.35440 & 22.7730 \\
\hline
\end{tabular}




\begin{tabular}{|c|c|c|c|c|c|c|c|c|c|c|c|c|c|c|c|c|}
\hline \multicolumn{3}{|c|}{ Date } & \multicolumn{3}{|c|}{ Time } & Latitude & Longitude & Depth & Mw & Mrr & Mrt & Mrp & Mtp & Mtt & Mpp & VarRed \\
\hline 1993 & 09 & 18 & 05 & & 27.20 & 36.4800 & 71.8000 & 117.6479 & 6.13179 & 9.52090 & -7.49970 & 8.08050 & -9.22810 & 6.15690 & -15.67790 & 13.2121 \\
\hline 1993 & 09 & 29 & 22 & 25 & 48.50 & 18.1100 & 76.5500 & 13.7623 & 6.08362 & 15.99240 & -2.02640 & -6.40700 & 5.02350 & -12.48880 & -3.50360 & 35.2837 \\
\hline 1993 & 12 & 30 & 14 & 24 & 5.30 & 44.9500 & 78.7600 & 18.4446 & 5.30448 & 0.81030 & -0.04570 & 0.60780 & -0.27110 & -1.00600 & 0.19580 & 55.4319 \\
\hline 1994 & 02 & 23 & 08 & 02 & 5.30 & 30.8300 & 60.5000 & 12.3478 & 6.06498 & 14.50360 & -5.37470 & 5.09370 & 5.97330 & -7.26140 & -7.24220 & 33.4309 \\
\hline 1994 & 02 & 24 & 00 & 11 & 12.80 & 30.7300 & 60.5200 & 11.3971 & 6.20186 & 23.71000 & -5.06320 & 0.89210 & 13.73340 & -10.29290 & -13.41720 & 44.6078 \\
\hline 1994 & 02 & 26 & 02 & 31 & 11.60 & 30.5900 & 60.4400 & 13.2090 & 5.96225 & 8.03130 & -5.44160 & 4.16310 & 5.09350 & -3.09530 & -4.93600 & 45.3430 \\
\hline 1994 & 02 & 28 & 11 & 13 & 54.10 & 30.7700 & 60.4800 & 12.4327 & 5.61215 & 2.93190 & -1.32840 & 1.26600 & 1.01680 & -1.63000 & -1.30200 & 13.3625 \\
\hline 1994 & 03 & 01 & 03 & 49 & 1.30 & 28.7500 & 52.4200 & -2.1624 & 5.99434 & -6.09580 & -1.97730 & -0.05400 & 1.14130 & -7.87600 & 13.97170 & 8.9364 \\
\hline 1994 & 03 & 30 & 19 & 55 & 44.10 & 28.9600 & 52.6000 & 19.8469 & 5.31265 & -0.19440 & -0.21890 & -0.41370 & 0.58630 & -0.78750 & 0.98190 & 6.6397 \\
\hline 1994 & 04 & 11 & 11 & 20 & 22.20 & 11.8200 & 43.1500 & 10.7326 & 5.77236 & -3.03700 & -1.96890 & -1.32120 & -0.09150 & 6.02880 & -2.99180 & 58.6395 \\
\hline 1994 & 05 & 01 & 12 & 00 & 37.00 & 37.1000 & 66.8500 & 25.4927 & 5.96427 & 7.59260 & -0.86970 & -2.41080 & 2.58340 & -12.00270 & 4.41010 & 44.3478 \\
\hline 1994 & 06 & 20 & $0 \overline{9}$ & 09 & 4.00 & 29.0600 & 52.4400 & 9.1436 & 5.85157 & -2.53420 & 2.60820 & $-\overline{3} .55990$ & 5.12920 & -1.23180 & 3.76600 & 19.5429 \\
\hline 1994 & 06 & 30 & 09 & 23 & 21.60 & 36.3400 & 71.0000 & 248.4365 & 6.27160 & 33.91650 & 0.54180 & 8.58620 & -7.71880 & -10.77020 & -23.14620 & 4.8465 \\
\hline 1994 & 07 & 01 & 10 & 12 & 41.30 & 40.0100 & 1300 & 50.7896 & 5.50583 & 1.52830 & 1.39470 & -0.33880 & -0.37620 & -1.88860 & 0.36020 & 27.6445 \\
\hline 1994 & 07 & 01 & 19 & 50 & 4.00 & 40.1 & 700 & & 5.07280 & 0.36700 & 0.21440 & 0.02380 & 0.04800 & & 0.15140 & \\
\hline 1994 & 07 & 24 & 14 & 47 & 48.60 & 37.2700 & 71.3100 & 114.7024 & 5.17731 & -0.31700 & -0.27090 & -0.47510 & -0.11530 & 0.54800 & -0.23100 & 23.9903 \\
\hline 1994 & 10 & 25 & 00 & 54 & 34.60 & 36.2500 & 71.0000 & 251.6328 & 5.95306 & 11.08710 & 2.55360 & 2.25880 & -0.15980 & -8.82370 & -2.26340 & 31.4187 \\
\hline 1995 & 02 & 23 & 21 & 03 & 2.00 & 35.0200 & 32.4400 & 14.0548 & 5.72570 & 2.46970 & -0.09260 & 4.06620 & -1.51680 & -0.59300 & -1.87670 & 63.2053 \\
\hline 1995 & 05 & 16 & 03 & 35 & 3.00 & 36.6700 & 00 & 185.8072 & 5.87960 & 5.35870 & -6.33940 & -1.40120 & -1.35720 & -4.54040 & -0.81840 & 35.5086 \\
\hline 1995 & 08 & 17 & 23 & 14 & 19.40 & 200 & & 244 . & 5.67061 & 3.0 & -1.06970 & & -1.23610 & 390 & 80 & $\$ 25$ \\
\hline 1995 & 10 & 01 & 15 & 57 & 16.00 & 38. & 00 & 13. & 6.30778 & -32.53460 & 14.72950 & -12.18040 & -12.95660 & 17.44430 & 15.09030 & 31. \\
\hline 1995 & 10 & 08 & 08 & 55 & 49.90 & 41. & 00 & 13.2383 & 5.66908 & 4.2 & 0.8 & 2150 & -0.29090 & -3.31650 & -0.92180 & \\
\hline 1995 & 10 & 18 & 09 & 30 & 38.60 & 300 & & 235.7519 & 6.26925 & 26.8 & 490 & -6.5 & -1.91800 & 4160 & 2630 & 6177 \\
\hline 1995 & 11 & 22 & 04 & 15 & 11.70 & 29.0700 & 34.7300 & 18.0244 & 6.95785 & -50.16700 & 220.02400 & 25.50020 & -203.61800 & -136.31200 & 186.47900 & \\
\hline 1995 & 12 & 05 & 18 & 49 & 33.80 & 39.4100 & 700 & 14.4265 & 5.64817 & 0.18970 & -0.00730 & -2.29770 & 0.65460 & -2.96070 & 7090 & 41.1483 \\
\hline 1996 & 03 & 28 & 07 & 28 & 28.10 & 12.0 & & 8.9 & 6.02864 & -2.4 & & -6.02080 & 1150 & 810 & 9.16620 & 21.4208 \\
\hline 1996 & 04 & 01 & 08 & 08 & 2.60 & 31.3 & 200 & 94.4 & 5.21135 & 0.7 & -0.2 & -0.03020 & -0.22480 & 3480 & 0.04920 & 7.6111 \\
\hline 1997 & 01 & 09 & 13 & 43 & 31.50 & 41. & & & 5.65829 & & & & -0. & & -0.13550 & 43.8882 \\
\hline 1997 & 02 & 04 & 10 & 37 & 47.10 & & 00 & & 6.44140 & 900 & & -2 . & 27. & -43.22990 & 49.24890 & 7.4118 \\
\hline 1997 & 02 & 27 & 21 & 08 & 2.30 & & 000 & & & 4800 & 3200 & 41. & 790 & -134.01000 & 780 & 9467 \\
\hline 1997 & 02 & 28 & 12 & 57 & 18.60 & & & & & & & & -7.8 & & & \\
\hline 1997 & 03 & 20 & 08 & 50 & 40.30 & & & & & -0 . & & & & & 90 & 190 \\
\hline 1997 & 04 & 11 & 05 & 34 & 42.70 & & & & & -8 & & -7. & & -2.3 & 7790 & 652 \\
\hline 1997 & 04 & 19 & 05 & 53 & 14.10 & & & & & & & -0.5 & -0.47530 & -1.90160 & 0.82090 & 692 \\
\hline 1997 & 05 & 10 & 07 & 57 & 29.70 & & & & & & & & & & 128.70100 & \\
\hline 1997 & 05 & 13 & 14 & 13 & 45.70 & & & 187. & & & & & -13 & -48 & 820 & 15.2089 \\
\hline 1997 & 09 & 07 & 10 & 15 & 24.90 & & & & & & & & & & & \\
\hline 1997 & 10 & 20 & 06 & 09 & 4.30 & 27. & & & 5.21 & & & & & & -0.00930 & 67.8401 \\
\hline 1998 & 02 & 20 & 12 & 18 & 6.20 & 36.5 & 800 & 241.8543 & 6.35917 & 26. & -2.88230 & 9380 & & -2.18750 & -24.49570 & 10.8756 \\
\hline 1998 & 03 & 14 & 19 & 40 & 27.00 & & & & & & -32.27800 & & & & 45.74980 & \\
\hline 1998 & 03 & 21 & 18 & 22 & 28.50 & 36.4 & & 233.3 & & & 6.780 & 760 & -0.28920 & -3.79470 & -0.02310 & 3.1293 \\
\hline 1998 & 05 & 29 & 22 & 49 & & & & & & & & & & & & \\
\hline & 06 & 27 & 13 & 55 & 52.10 & & & & & & & & & -21.14380 & 380 & 33.8143 \\
\hline 1998 & 07 & 09 & 14 & 19 & 18.40 & & & & & & & & & & & \\
\hline 1998 & 08 & 02 & 04 & 40 & 46.50 & & 300 & 13. & & -1. & -0.14400 & -1.81870 & 0.89730 & & 1.80530 & 37.9 \\
\hline 1998 & 08 & 27 & 09 & 03 & 36.70 & 39.5100 & 77.2200 & 27.0066 & 6.28624 & -4.76420 & -0.37420 & -6.47610 & 13.03340 & -27.88060 & 32.64480 & 10.8028 \\
\hline 1999 & 03 & 28 & 19 & 05 & 11.00 & 30.3800 & 79.2100 & 14.7657 & 6.31314 & 11.99260 & 29.34690 & -18.05310 & 6.97820 & -11.89840 & -0.09420 & 57.4404 \\
\hline
\end{tabular}




\begin{tabular}{|c|c|c|c|c|c|c|c|c|c|c|c|c|c|c|c|c|}
\hline $\mathrm{Da}$ & ate & & & Tin & & Latitude & Longitude & Depth & $\mathrm{Mw}$ & Mrr & Mrt & Mrp & Mtp & Mtt & Mpp & VarRed \\
\hline 1999 & 05 & 06 & 23 & 00 & 53.10 & 29.3400 & 52.0300 & 11.6472 & 6.17716 & -0.44410 & 0.06500 & 4.33800 & 6.50550 & -21.63170 & 22.07580 & 3.7609 \\
\hline 1999 & 06 & 29 & 23 & 18 & 5.60 & 36.5000 & 70.9600 & 187.3612 & & 4.79390 & -0.32650 & & -0.41360 & -2.97270 & -1.82120 & 12.7162 \\
\hline 1999 & 09 & 13 & 11 & 55 & 28.20 & 40.3100 & 30.2900 & 15.2455 & 5.69743 & 0.76350 & 0.05480 & 2.57420 & -3.49790 & 0.16570 & -0.92910 & 32.3675 \\
\hline 1999 & 11 & 08 & 16 & 45 & 43.00 & 36.4800 & 70.8100 & 234.6247 & 6.51939 & 65.66450 & 14.62950 & 38.84010 & -15.99610 & -10.07460 & -55.58990 & 15.7491 \\
\hline 1999 & 11 & 08 & 21 & 37 & 23.20 & 36.0400 & 61.3800 & 49.6111 & 5.48349 & 1.95220 & 0.18010 & -0.13470 & 1.12440 & -1.52250 & -0.42970 & 16.4237 \\
\hline 1999 & 11 & 12 & 16 & 57 & 19.50 & 40.9300 & 31.2500 & 19.0916 & 7.04743 & -69.78320 & -30.21440 & 201.75300 & -416.23000 & 72.57320 & -2.79020 & 30.3440 \\
\hline 2000 & 01 & 26 & 23 & 00 & 19.90 & 40.2700 & 52.7300 & 63.1345 & 5.11978 & 0.16020 & 0.31310 & 0.22050 & 0.44340 & -0.07580 & -0.08440 & 34.4503 \\
\hline 2000 & 03 & 05 & 09 & 40 & 6.10 & 27.6100 & 56.4000 & 29.3443 & 5.27512 & 0.66110 & 0.03030 & 0.19760 & 0.14340 & -1.15020 & 0.48910 & 22.5114 \\
\hline 2000 & 04 & 20 & 08 & 41 & 29.60 & 38.7400 & 66.1400 & 12.1899 & 5.17355 & 0.54410 & -0.40440 & -0.02840 & -0.35460 & -0.15600 & -0.38810 & 36.2738 \\
\hline 2000 & 05 & 12 & 23 & 10 & 30.00 & 36.0400 & 70.5300 & 90.5959 & 6.21401 & -3.18320 & 21.69790 & 12.81930 & 3.67960 & 7.81900 & -4.63580 & 15.1032 \\
\hline 2000 & 06 & 06 & 02 & 41 & 49.80 & 40.7500 & 32.7000 & 13.5852 & 5.98778 & -6.65960 & -4.14730 & 3.91330 & -8.17740 & -0.27820 & 6.93790 & 3.7347 \\
\hline 2000 & 07 & 17 & 22 & 53 & 47.30 & 36.2400 & 70.8200 & 137.5000 & 6.36987 & 14.26550 & 6680 & 15.51280 & 5.16900 & -26.34650 & 12.08100 & \\
\hline 2000 & 11 & 25 & 18 & 09 & 11.40 & 40.2400 & 49.9500 & 15.5295 & 6.23929 & 2.92750 & 21.06170 & -19.23440 & -1.22520 & 1.03320 & -3.96070 & 87.8998 \\
\hline 2000 & 11 & 25 & 18 & 10 & 47.40 & & & & & -28.63140 & & -13.00900 & & & & \\
\hline 2000 & 12 & 06 & 17 & 11 & 6.40 & 39.6000 & 54.8700 & 33.6362 & 6.82088 & 129.67100 & 135.66000 & 43.90150 & 92.45500 & -131.89400 & 2.22290 & 65.4717 \\
\hline 2001 & 01 & 26 & 03 & 16 & 40.50 & 23.6300 & 400 & 932 & 7.42519 & 1162.82000 & 616.94500 & 646.71300 & 190.26300 & -1649.20000 & 486.37200 & 69.1265 \\
\hline 2001 & 01 & 28 & 01 & 02 & 10.70 & 23.6 & 00 & 13. & 869 & 3.93130 & & 1.1 & 0.63700 & -4.18910 & 0.25770 & \\
\hline 2001 & 02 & 25 & 02 & 21 & 59.60 & 36.4 & 200 & 190.9629 & 260 & $21 .($ & 370 & 7.0 & 820 & -20.92250 & 1450 & 012 \\
\hline 2001 & 02 & 25 & 02 & 21 & 59.60 & 00 & 6200 & 190. & 315 & 21 . & 270 & & 740 & 0900 & 520 & \\
\hline 2001 & 06 & 10 & 01 & 52 & 8.00 & 100 & 800 & 206 & & & 650 & 0.0 & 940 & 3350 & -0.17200 & 805 \\
\hline 2001 & 06 & 15 & 16 & 19 & 7.60 & 14.0300 & 51.5900 & 348 & 5.78 & 0.79 & & -2.1 & & & -4.59950 & 29.1867 \\
\hline 2001 & 11 & 23 & 20 & 43 & 3.50 & 36.4300 & 71.5000 & 93.6 & $.0287 \overline{5}$ & 10.18920 & 9670 & 4.70330 & -9.58010 & 7080 & -3.91850 & 9.2644 \\
\hline 2002 & 01 & 03 & 07 & 05 & 27.70 & 35.7100 & 00 & 126.2346 & 0707 & 3960 & 3950 & 5.61080 & -5.8 & 3820 & -6.15150 & 13.3020 \\
\hline 2002 & 02 & 03 & 09 & 26 & 43.30 & 300 & & 14.1723 & & -3.5 & & -1.0 & 1.5 & & 2.80180 & 428 \\
\hline 2002 & 02 & 17 & 13 & 03 & 52.70 & 000 & 00 & 42.6 & & 090 & 7490 & 0.30060 & 0.30340 & -0.37900 & 0.00810 & 33.7267 \\
\hline 2002 & 03 & 03 & 12 & 08 & 7.80 & 36.5700 & & 224 . & 7.3 & 9000 & 1000 & -350.10200 & 171.34700 & -806.29800 & -70.49400 & 160 \\
\hline 2002 & 03 & 03 & 12 & 08 & 7.80 & & & 224 . & & 600 & 2000 & -346 & 170.2 & 9600 & 9200 & 664 \\
\hline 2002 & 03 & 25 & 14 & 56 & 33.80 & 300 & & 330 & & & 80 & -2.77380 & -3.77680 & & -14.34440 & 637 \\
\hline 2002 & 03 & 27 & 08 & 52 & 52.3 & & & & & & & & & & & \\
\hline 2002 & 04 & 12 & 04 & 00 & 23.70 & 600 & & & & & & & 660 & 1.7 & 10 & 10.6627 \\
\hline 2002 & 06 & 22 & 02 & 58 & 21.30 & 200 & & & & 52. & 150 & -7.0 & $13 .($ & -43 & 40 & 553 \\
\hline 2002 & 08 & 13 & 08 & 37 & 22.80 & 100 & 700 & & & & & & & & & 946 \\
\hline 2002 & 09 & 25 & 22 & 28 & 11.90 & 32.0400 & & & & & -0 . & -0.0 & & & & 49 \\
\hline 2002 & 11 & 20 & 21 & 32 & 30.80 & & & & & & & & & & & \\
\hline 2002 & 12 & 25 & 12 & 57 & 3.20 & & & & & & & & & & 1.26560 & \\
\hline 2003 & 01 & 14 & 14 & 13 & 57.20 & 00 & 00 & & & & & & & & -0.02270 & 977 \\
\hline 2003 & 01 & 27 & 05 & 26 & 23.00 & & & & & & 270 & -1.5 & & -7.2 & 6980 & \\
\hline 2003 & 02 & 24 & 02 & 03 & 41.50 & & 77.2400 & 27.2 & & -2.1 & & & & -17.3 & 19.50500 & 37 \\
\hline 2003 & 03 & 12 & 04 & 47 & 51.30 & & & & & & & & & & & \\
\hline 2003 & 05 & 01 & 00 & 27 & 4.70 & 39.0400 & 300 & 13.0001 & & & 20 . & & 15.6 & -24.6 & 22.62150 & 13.1436 \\
\hline & 05 & 22 & 18 & 11 & 57.00 & & & & & & & & & & 0.94460 & \\
\hline 2003 & 05 & 24 & 01 & 46 & 6.30 & & & & & -1.3 & -0.3 & -1.17890 & & 3.0 & -1.66150 & 45.2048 \\
\hline 2003 & 07 & 10 & 17 & 06 & 37.70 & & & & & & & -0.1 & & & & \\
\hline 2003 & 07 & 10 & 17 & 40 & 15.90 & 2600 & 54. & & 5.6 & & & 500 & & -3.02020 & 3730 & \\
\hline & 08 & 21 & 04 & 02 & 9.20 & 28.8800 & & & & & & & & & 390 & \\
\hline 2003 & 09 & 01 & 23 & 16 & 35.00 & & & & & & -0.1 & -2.0 & & & 320 & \\
\hline 2003 & 12 & 26 & 01 & 56 & 52.40 & 29.1000 & 58.2400 & 13.0828 & 6.52137 & 17.02420 & -45.63960 & -13.89160 & 57.40560 & -13.04800 & -3.97630 & 13.3779 \\
\hline
\end{tabular}




\begin{tabular}{|c|c|c|c|c|c|c|c|c|c|c|c|c|c|c|c|c|}
\hline & ate & & & Tim & ne & Latitude & Longitude & Depth & Mw & Mrr & Mrt & Mrp & Mtp & Mtt & Mpp & VarRed \\
\hline 2004 & 03 & 12 & 22 & 45 & 19.00 & 36.3800 & 70.6000 & 215.8230 & 5.66025 & 2.61090 & 2.77770 & 0.57920 & 0.04170 & -2.71860 & 0.10770 & 3.8555 \\
\hline 2004 & 05 & 28 & 12 & 38 & 44.30 & 36.5500 & 51.5800 & 21.6581 & 6.13541 & 16.03470 & -8.28180 & 7.24410 & 9.34560 & -9.63450 & -6.40020 & 63.2030 \\
\hline 2004 & 10 & 07 & 21 & 46 & 20.30 & 37.3700 & 54.3200 & 34.5108 & 5.57022 & 2.21780 & -1.03420 & 0.49950 & -1.76940 & -1.06970 & -1.14810 & 20.1789 \\
\hline 2004 & 10 & 31 & 06 & 02 & 59.00 & 35.2000 & 74.3700 & 5.7731 & 4.71642 & -0.08220 & 0.05550 & -0.09040 & 0.03180 & 0.11200 & -0.02980 & 71.1008 \\
\hline 2004 & 11 & 17 & 20 & 58 & 22.30 & 39.2700 & 71.7600 & 23.1076 & 5.70336 & 0.02810 & 0.36510 & -0.29490 & -4.37150 & -1.05800 & 1.02990 & 30.0738 \\
\hline 2005 & 02 & 14 & 23 & 38 & 8.70 & 41.7200 & 79.2700 & 28.5275 & 5.86564 & 1.40190 & 7.28690 & 2.22690 & 0.70530 & -2.31950 & 0.91760 & 55.0032 \\
\hline 2005 & $0 \overline{2}$ & 22 & 02 & 25 & 22.80 & 30.7600 & 56.8100 & 7.7923 & 6.34613 & 39.85900 & -6.76470 & 2.28370 & -11.11940 & -39.03910 & -0.81990 & $55.474 \overline{3}$ \\
\hline 2005 & 02 & 25 & 23 & 04 & 4.00 & 38.1800 & 72.4000 & 110.5242 & 5.63058 & -1.75140 & -2.34530 & -0.60670 & -2.04170 & 0.75710 & 0.99430 & 29.5035 \\
\hline 2005 & 03 & 13 & 03 & 31 & 23.10 & 26.7300 & 62.0000 & 57.2783 & 5.89361 & -7.94410 & -1.39350 & -0.37650 & 2.04900 & 8.70360 & -0.75950 & 28.7054 \\
\hline 2005 & 03 & 14 & 01 & 55 & 55.60 & 39.4400 & 700 & 6.7294 & 5.68366 & -1.35140 & 1.75330 & 1.48590 & -2.95100 & -0.89370 & 2.24510 & 57.0533 \\
\hline 2005 & 05 & 14 & 18 & 04 & 55.10 & 30.7200 & 56.8400 & 5.4389 & 823 & 0.66370 & -0.02340 & 0.30960 & 0.08480 & -0.66190 & -0.00180 & 703 \\
\hline 2005 & 08 & 25 & 21 & 08 & 13.00 & 37.1300 & 79.3500 & 14.5293 & 5.26148 & 0.70610 & -0.59470 & 0.23930 & 0.14850 & -0.75040 & 0.04430 & 1363 \\
\hline 2005 & 08 & 26 & 18 & 16 & 33.60 & 14.5700 & 51.9600 & 5.5768 & & -1.99050 & -1.13030 & 2.89320 & 8.32490 & 9.43820 & -7.44770 & \\
\hline 2005 & 10 & 08 & 03 & 50 & 40.80 & 34.3800 & 73.47 & 11.3705 & 7.42803 & 1732.71000 & 563.36700 & 188.28300 & 665.03000 & -907.31700 & -825.39800 & 74.7801 \\
\hline 2005 & 10 & 08 & 10 & 46 & 28.80 & 000 & & 9.4926 & 6.37960 & 43.21810 & 21.98390 & -0.67480 & 16.94800 & -18.47480 & -24.74330 & \\
\hline 2005 & 10 & 08 & 12 & 25 & 20.20 & 34.6 & 400 & 15.6467 & & 3.21830 & -0.18670 & 1.35440 & 1.03970 & -2.22100 & -0.99730 & 091 \\
\hline 2005 & 10 & 08 & 21 & 13 & 31.90 & 34.6 & 73.1000 & & 5.44767 & 1.66590 & 9990 & -0.31220 & 000 & 0.14220 & -1.80820 & 27.4051 \\
\hline 2005 & 10 & 08 & 21 & 45 & 9.80 & 000 & & 106 & 328 & 0.90660 & & -0.13410 & 60 & -0.38580 & -0.52080 & 836 \\
\hline 2005 & 10 & 09 & 07 & 09 & 18.60 & 34. & 00 & 130 & 5.2 & 0.38480 & 70 & 1030 & 0.38670 & -0.21170 & -0.17310 & 22.7327 \\
\hline 2005 & 10 & 09 & 08 & 30 & 1.10 & & & 10. & & & & -0.1 & & 90 & 4070 & \\
\hline 2005 & 10 & 09 & 19 & 20 & 37.40 & 34 . & 300 & 69 & 5.3 & 000 & 57380 & 0.18120 & 20 & 8000 & -0.04000 & 17.2004 \\
\hline 2005 & 10 & 12 & 20 & 23 & 38.20 & 34 . & & 11.9 & & 0.8 & -0.0 & 400 & & -0. & 1000 & 579 \\
\hline 2005 & 10 & 19 & 02 & 33 & 28.30 & & & & & & & 90 & & & 80 & \\
\hline 2005 & 10 & 19 & $0 \overline{3}$ & 16 & 21.10 & & & & & & & 620 & & -0. & 570 & \\
\hline 2005 & 10 & 23 & 15 & 04 & 20.90 & & & & & & & & & & & \\
\hline & 11 & 27 & 10 & 22 & 19.20 & 00 & & 629 & & & & 3660 & -2.3 & 9190 & -1.33680 & 003 \\
\hline 2005 & 12 & 12 & 21 & 47 & 46.10 & 36.4500 & 71.0600 & 211.9298 & 6.58734 & 90.6 & 20.5 & 15.28820 & 2.22880 & 6360 & 2.99900 & 6.9747 \\
\hline 2006 & 02 & 28 & 07 & 31 & 2.70 & 27.8 & & 809 & & & & & & & 1.03100 & 44.3984 \\
\hline 2006 & 03 & 25 & 07 & 28 & 57.70 & & & 374 & & & & -0. & -0.2 & & 030 & 753 \\
\hline 2006 & 03 & 31 & 01 & 17 & 1.00 & & & & & & & & & & 270 & \\
\hline 06 & 04 & 06 & 17 & 59 & 16.40 & & & & & & & -0.4 & & & 500 & \\
\hline & 06 & 28 & 21 & 02 & 9.20 & & & & & & & & & & 000 & 696 \\
\hline & 07 & 06 & 03 & 57 & 53.50 & & & & & & & & -2.4 & & 470 & 777 \\
\hline 2006 & 09 & 11 & 18 & 12 & 22.30 & & & & & -0. & & -0.19030 & & & & \\
\hline 2006 & 10 & 12 & 17 & 08 & 20.40 & & & & & & & & & & & \\
\hline 2006 & 12 & 25 & 20 & 01 & 0.40 & & & 19.0257 & 5.67761 & & & 8350 & -2.20330 & 1380 & 0.23330 & 82.7551 \\
\hline 2006 & 12 & 30 & 08 & 30 & 49.80 & & & & & -11 . & & & & 4720 & -38.47360 & 45.5868 \\
\hline 2007 & 01 & 08 & 17 & 21 & 50.30 & & & & & & & & & & & \\
\hline & 01 & 17 & 23 & 18 & 50.10 & & & & & & & & & & -13.01110 & \\
\hline 2007 & 02 & 21 & 11 & 05 & 29.20 & & & 8.9387 & & -2.31400 & & -2.60530 & 1.58560 & & 1.78430 & 52.0490 \\
\hline 2007 & 04 & 03 & 03 & 35 & 7.30 & 36. & & 138.3371 & 3897 & 17.03130 & 12.53830 & -15.07310 & -2.49220 & 6.23910 & 7040 & 3.4917 \\
\hline & 06 & 18 & 14 & 29 & 48.30 & & & 17.4206 & & & & -0.72620 & & -1.07 & 0.09610 & 54.7782 \\
\hline & 07 & 21 & 22 & 44 & 13.60 & 38.9800 & 70.1700 & 8.4526 & 5.12645 & 0.31380 & 0.35230 & -0.08030 & -0.39800 & -0.02760 & -0.28620 & 18.6513 \\
\hline
\end{tabular}


$\begin{array}{llllllllll}\text { Date } & \text { Time Latitude Longitude Depth Mw } & \text { Mrr } & \text { Mrt } & \text { Mrp } & \text { Mtp } & \text { Mtt } & \text { Med }\end{array}$

Table 1.2: Earthquake catalog containing 184 event solutions solved for using a 3D moment tensor inversion method Liu et al. (2004). Results are azimuthally weighted, 7-parameter (Mij and depth), zero-trace solutions. Mij components are normalized by dividing by $1 x 10^{17} \mathrm{~N} . \mathrm{m}$. 


\section{BIBLIOGRAPHY}

Adams, A., R. Brazier, A. Nyblade, A. Rodgers, and A. Al-Amri, Source Parameters for Moderate Earthquakes in the Zagros Mountains with Implications for the Depth Extent of Seismicity, Bulletin of the Seismological Society of America, $99(3), 2044,2009$.

Bassin, C., G. Laske, and G. Masters, The current limits of resolution for surface wave tomography in north america, EOS Trans. AGU., 81(F897), 2000.

Covellone, B. M., and B. Savage, A quantitative comparison between $1 \mathrm{~d}$ and $3 \mathrm{~d}$ source inversion methodologies: Application to the middle east, Bulletin of the Seismological Society of America, 102(5), 2189-2199, 2012.

DeMets, C., R. Gordon, D. Argus, and S. Stein, Effect of recent revisions to the geomagnetic reversal rimescale on estimates of current plate motions, Geophysical Research Letters, 21, 2191-2194, 1994.

Dziewonski, A., and D. Anderson, Preliminary reference Earth model, Physics of the Earth and Planetary Interiors, 25(4), 297-356, 1981.

Dziewonski, A., T. Chou, and J. Woodhouse, Determination of earthquake source parameters from waveform data for studies of global and regional seismicity, Journal of Geophysical Research, 86(B4), 2825-2852, 1981.

Hatzfeld, D., and P. Molnar, Comparisons of the kinematics and deep structures of the Zagros and Himalaya and of the Iranian and Tibetan plateaus and geodynamic implications, Reviews of Geophysics, 48(2), 2010.

Henry, C., J. Woodhouse, and S. Das, Stability of earthquake moment tensor 
inversions: effect of the double-couple constraint, Tectonophysics, 356(1-3), 115$124,2002$.

Hjörleifsdóttir, V., and G. Ekström, Effects of three-dimensional earth structure on cmt earthquake parameters, Physics of the Earth and Planetary Interiors, $179(3-4), 178-190,2010$.

Hollingsworth, J., J. Jackson, R. Walker, M. Gheitanchi, and M. Bolourchi, Strikeslip faulting, rotation, and along-strike elongation in the Kopeh Dagh mountains, NE Iran, Geophysical Journal International, 166(3), 1161-1177, 2006.

Hollingsworth, J., J. Jackson, R. Walker, and H. Nazari, Extrusion tectonics and subduction in the eastern South Caspian region since $10 \mathrm{Ma}$, Geology, 36(10), $763,2008$.

Hollingsworth, J., J. Jackson, R. Walker, and H. Nazari, Extrusion tectonics and subduction in the eastern South Caspian region since 10 Ma: Reply, Geology, $37(12), \mathrm{e} 199,2009$.

Holt, W., J. Ni, T. Wallace, and A. Haines, The active tectonics of the eastern Himalayan syntaxis and surrounding regions, Journal of Geophysical ResearchSolid Earth, 96(B9), 14,595-14,632, 1991.

Jackson, J., K. Priestley, M. Allen, and M. Berberian, Active tectonics of the South Caspian basin, Geophysical Journal International, 148(2), 214-245, 2002.

Julian, B., A. Miller, and G. Foulger, Non-double-couple earthquakes 1. theory, Reviews of Geophysics, 36(4), 525-549, 1998.

Komatitsch, D., and J. Tromp, Introduction to the spectral element method for three-dimensional seismic wave propagation, Geophysical Jounrnal International, $139(3), 806-822,1999$. 
Komatitsch, D., and J. Tromp, Spectral-element simulations of global seismic wave propagation-I. Validation, Geophysical Journal International, 149(2), 390-412, 2002a.

Komatitsch, D., and J. Tromp, Spectral-element simulations of global seismic wave propagation-II. Three-dimensional models, oceans, rotation and self-gravitation, Geophysical Journal International, 150(1), 303-318, $2002 b$.

Kustowski, B., G. Ekstrom, and A. Dziewonski, The shear-wave velocity structure in the upper mantle beneath Eurasia, Geophysical Journal International, $174(3)$, 978-992, 2008.

Liu, Q., J. Polet, D. Komatitsch, and J. Tromp, Spectral-element moment tensor inversions for earthquakes in southern California, Bulletin of the Seismologiacl Society of America, 94(5), 1748-1761, 2004.

Maggi, A., C. Tape, M. Chen, D. Chao, and J. Tromp, An automated time-window selection algorithm for seismic tomography, Geophysical Journal International, $178(1), 2009$.

Miller, A., G. Foulger, and B. Julian, Non-double-couple earthquakes. 2. Observations, Reviews of Geophysics, 36(4), 551-568, 1998.

Muyzert, E., and R. Snieder, The influence of errors in source parameters on phase velocity measurements of surface waves, Bulletin of the Seismological Society of America, 86(6), 1863-1872, 1996.

NOAA, Data Announcement 88-MGG-02. Digital relief of the surface of the Earth, National Oceanographic and Atmospheric Administration, National Geophysical Data Center, Boulder, Colorado, 1988. 
Press, W., S. Teukolsky, W. T. Vetterling, and B. P. Flannery, Numerical Recipes in $C$, 2nd ed., Cambridge University Press, 1997.

Reigber, C., et al., New space geodetic constraints on the distribution of deformation in Central Asia, Earth and Planetary Science Letters, 191 (1-2), 157-165, 2001.

Stein, S., and M. Wysession, An introduction to seismology, earthquakes, and earth structure, Wiley-Blackwell, 2009.

Talebian, M., and J. Jackson, A reappraisal of earthquake focal mechanisms and active shortening in the Zagros mountains of Iran, Geophysical Journal International, 156(3), 506-526, 2004.

Tape, C., Q. Liu, A. Maggi, and J. Tromp, Adjoint tomography of the southern California crust, Science, 325 (5943), 988, 2009.

Tatar, M., D. Hatzfeld, and M. Ghafory-Ashtiany, Tectonics of the Central Zagros (Iran) deduced from microearthquake seismicity, Geophysical Journal International, 156(2), 255-266, 2004.

Vernant, P., et al., Present-day crustal deformation and plate kinematics in the Middle East constrained by GPS measurements in Iran and northern Oman, Geophysical Journal International, 157(1), 381-398, 2004.

Wessel, P., and W. Smith, Free software helps map and display data, Eos Trans. AGU, 72(441), 445-446, 1991.

Zhao, L., T. Jordan, K. Olsen, and P. Chen, Fréchet kernels for imaging regional earth structure based on three-dimensional reference models, Bulletin of the Seismological Society of America, 95(6), 2066, 2005. 


\title{
CHAPTER 2 \\ Wave speed structure of the Ontong Java Plateau
}

\author{
by \\ Brian M. Covellone ${ }^{1}$; Brian Savage ${ }^{1,2}$; Yang Shen ${ }^{1}$ \\ Prepared for submission to Earth and Planetary Science Letters. \\ ${ }^{1}$ Graduate School of Oceanography, University of Rhode Island, Narragansett, RI 02882 \\ ${ }^{2}$ Department of Geosciences, University of Rhode Island, Kingston, RI 02881
}




\section{Abstract}

The Ontong Java Plateau (OJP) represents the result of a significant event in the Earth's geologic history. Limited geophysical and geochemical data, as well as the plateau's relative isolation in the Pacific ocean, have made interpretation of the modern day geologic structure and its 120 Ma formation history difficult.

Here we the present highest resolution image to date of the wave speed structure of the OJP region. We use a unique data set that combines ambient noise and earthquake waveforms and an iterative finite-frequency tomography methodology. The uniqueness and combination of datasets allow us to best exploit the limited station distribution in the Pacific and image wave speed structures between $35 \mathrm{~km}$ and greater than $250 \mathrm{~km}$ into the Earth.

We image a region of fast shear wave speeds, greater than $4.75 \mathrm{~km} / \mathrm{s}$, that extends to greater than $100 \mathrm{~km}$ beneath the plateau. The wave speeds are similar to as observed in cratonic environments and are consistent with a compositional anomaly likely a result of eclogite entrainment during the plateau's formation.

The combination of our imaged wave speed structure and previous geochemical work, specifically enrichment in siderophile elements, suggest that a surfacing plume head entrained eclogite from the deep mantle accounts for the anomalous buoyancy characteristics of the plateau and observed fast wave speeds.

\section{Introduction}

The Ontong Java Plateau (OJP) represents the largest preserved Large Igneous Province (LIP) by volume on the Earth (Coffin and Eldholm, 1994; Gladczenko et al., 1997; Ito and van Keken, 2007). At the surface, the OJP's area of $2 \mathrm{Mkm}^{2}$ also makes it the largest oceanic plateau (Gladczenko et al., 1997). Taylor (2006) showed that the OJP, Manihiki Plateau (MP) and Hikurangi Plateau (HP) were once part of the same feature and were subsequently separated by seafloor spread- 
ing during the Cretaceous. Including the $\mathrm{MP}$ and $\mathrm{HP}$ as originating from the same edifice, greater than $4 \mathrm{Mkm}^{2}$ of ocean floor has been effected by the massive outpouring of material associated with the three plateau's formation (Ito and van Keken, 2007). Connections with the Louisville Hotspot Chain have also been made, suggesting that the chain represents a plume tail (Neal et al., 1997; Chandler et al., 2012) to the OJP's surfaced plume head. This major event in Earth's history is a result of a process that is radically different and not currently active, and would results in rapid and catastrophic global environmental change. Estimates of the volume of magma erupted range from 44 to $57 \mathrm{Mkm}^{3}$, over a geologically short time period, 6 - 14 Myrs (Coffin and Eldholm, 1994; Gladczenko et al., 1997; Tejada et al., 2002); for comparison, Crisp (1984) estimates current global volcanism rates of 26 to $34 \mathrm{~km}^{3} \mathrm{yr}^{-1}$. Despite its apparent significance to Earth's geologic history, knowledge of the OJP is still under developed.

Current understanding of the OJP comes from a broad range of research. Sampling of the OJP's surface has been done using geochemistry and petrology on recovered rock samples from DSDP and ODP cruise legs as well as sampling on nearby islands in the Solomon chain (Neal et al., 1997; Michael, 1999; Tejada et al., 1996, 2002, 2004). Crust and upper mantle structure has been investigated using gravity and magnetic surveys (Nakanishi et al., 1992; Gladczenko et al., 1997) as well as active-source seismic profiling (Furumoto et al., 1976; Hussong et al., 1979). Deeper seismic structures have been imaged using Rayleigh-wave seismic tomography (Richardson et al., 2000), seismic attenuation (Gomer and Okal, 2003) and anisotropy (Klosko et al., 2001).

The result of these studies paint a complex geologic history spanning over 120 million years (Ma) and despite previous work, a consensus regarding the genesis of the OJP is lacking and several hypotheses have developed. Two main hypotheses on the OJP's origin invoke either 1) the surfacing of a buoyant plume head or 2) 
vigorous passive mantle upwelling at or near a spreading ridge, as responsible for the plateau's emplacement.

\section{Plume Source}

The prevailing mechanism for the origin of LIPs has been the decompression melting of a surfacing mantle plume head (Griffiths et al., 1989; Campbell, 1998). A Rayleigh-Taylor instability originating from the core-mantle boundary or the $660 \mathrm{~km}$ transition zone can be positively buoyant due to either a thermal or compositional anomaly compared to the ambient mantle (Olson, 1990; Larson and Kincaid, 1996; Campbell, 2005). The OJP, and other LIPs, would be a product of high degrees of melting requiring high mantle temperature anomalies that rise quickly and adiabatically through the mantle (Larson, 1991; Larson and Kincaid, 1996), resulting in widespread melting, drying and depletion of the mantle beneath a forming plateau (Hall and Kincaid, 2004). Larson (1991) suggested this excess of heat, originating from the core-mantle boundary, could alter normal mantle convection, changing the magnetic reversal frequency and leading to the observed mid-Cretaceous magnetic quite zone following the formation of the OJP.

The most compelling evidence for a plume source to the OJP is the volume and rate of erupted material. Erupted volume estimates range from 44 to $57 \mathrm{Mkm}^{3}$ over 6 - 14 Myrs (Coffin and Eldholm, 1994; Gladczenko et al., 1997; Tejada et al., 2002). Tejada et al. (1996, 2002) determine the main plateau forming event occurred around 120 Ma, with a smaller, but significant, volume of material emplaced around $90 \mathrm{Ma}$. Geochemically, samples represent high degrees of melting to a relatively homogeneous and well-buffered OIB-like source ( Tejada et al., 1996; Michael, 1999). Enrichment in siderophile elements, such as molybdenum (Mo), members of the platinum group, and gold (Au), may suggest a core-mantle boundary source, consistent with a plume hypothesis (Jain et al., 1996; Neal et al., 1997; Ely and Neal, 2003). 
Despite the evidence of a plume source for the OJP, complications arise when examining the emplacement depth and isostatic topography of the plateau. The vesicularity of the OJP lavas and presence of microfossils suggest that plateau emplacement was entirely submarine, at depths greater than $800 \mathrm{~m}$ below sea level (Mahoney et al., 2001). Korenaga (2005) suggested that based on a realistic geotherm for a mantle hot enough to induce melting, the plateau should have been emplaced at or above sea level based solely on the isostatic topography; the addition of a buoyant plume head would dynamically raise the plateau further. Using numerical models, Farnetani and Richards (1994), suggested uplift of approximately $5 \mathrm{~km}$ above abyssal sea floor when lithospheric extension is allowed in their model, similar to the pre-emplacement tectonic setting near the OJP. Hall and Kincaid (2004) suggested the formation of a viscous "plug" due to significant melt extraction and dehydration. Flow around the plug could result in high melt extraction rates and limited uplift; further the viscous plug would be more resistant to mantle flow and able to persistent for > 120 Ma. In addition to a lack of uplift, post-emplacement subsidence has been retarded relative to normal seafloor and seafloor adjacent to the plateau (Neal et al., 1997), suggesting a remnant positive buoyancy within the mantle beneath the OJP.

\section{Passive rift driven upwelling}

As an alternative to the plume-driven hypothesis, Korenaga (2005) proposed that entrainment of dense eclogite, by vigorous plate-driven mantle flow due to fast plate spreading rates, could explain both the topography and geochemistry of the OJP. Korenaga (2005) suggested the dense eclogite comes from recycled subducted crust. Initial formation of the combined plateaus occurred in the vicinity of the Tongareva triple junction (Pacific-Phoenix-Farallon); the Osbourn Trough separates the MP and HP, while spreading in the Ellice Basin seperated the OJP and MP (Larson, 1997; Billen and Stock, 2000; Viso et al., 2005; Taylor, 2006). 
Nearby magnetic lineations (M0-M7) imply a half spreading rate of $7.7 \mathrm{~cm} / \mathrm{yr}$ between 120 - 129Ma (Larson, 1997). Korenaga (2005) suggested that this rapid spreading rate alone should be large enough to entrain material denser than nominal mantle, and would only be enhanced by the presence of a nearby triple junction.

What follows is a description of our tomography methodology, using a unique data set combining ambient noise and earthquake waveforms. This allows us to obtain the resolution at depths in the crust and upper mantle necessary for interpretation of the OJP's wave speed structure and arrive at a hypothesis regarding its formation.

\section{Methodology}

To determine the 3-dimensional wave speed structure beneath the OJP we employed a two phase, iterative, tomography using full-waveform ambient noise and earthquake data. Due to the sparse coverage of seismic stations and earthquakes in the Pacific ocean and the relative isolation of the OJP, a two step process was used to image the seismic wave speed structure beneath the plateau. The first phase used Green's functions derived from ambient noise data at periods up to 200 seconds. This ambient noise-only model provided an improved base model for subsequent iterations using joint ambient noise and earthquake data. Use of Green's functions from ambient noise as a starting point effectively exploits the $20+$ years of continuous, broadband seismic data, and is not reliant on earthquake distributions and solving for earthquake source mechanisms. This eliminates a source of error, the earthquake mechanism and location, during the initial iterations and allows

the model converge on large scale features. Ambient noise further exploits small temporary seismic station deployments that may not gather sufficient earthquake data during their deployment window.

We added earthquake waveform data after changes in the wave speed structure 
converged using only ambient noise Green's functions. The use of both ambient noise and earthquake data is complementary as each data set samples different portions of the 3-dimensional model space. The addition of earthquakes tripled the number of total measurements used in the inversion and provided higher quality measurements than those strictly from ambient noise. The large magnitude sources associated with earthquake events produced more distinct surface wave signals with higher signal-to-noise ratios. The surface waves measured with both data sets have path coverage sensitive to crust and upper mantle $(<500 \mathrm{~km})$ Earth structure, and is key to the understanding of the OJP's wave speed structure and formation.

\section{Data Preparation}

To extract usable Rayleigh wave signals from continuous raw seismic data, we use an ambient noise processing procedure outlined in Shen et al. (2012) and Gao and Shen (2014). Continuous, vertical component data from 1990-2011 was gathered from IRIS DMC for 54 stations throughout the Pacific (Figure 2.1). The instrument response is removed and the data was cut into daily segments and resampled to 1 sample per second. A frequency time normalization (FTN), following Shen et al. (2012), is used to normalize the data; earthquakes larger than Mw 5.5 are then removed from the time series. Using the one day long records, a cross correlation between station pairs was then calculated with one station acting as a "virtual" source. The cross correlated records were then summed into monthly stacks; the total sum of these records represents our empirical Green's Function (EGF) following a time derivative (Figure 2.10). Monthly stacks are computed to quantify the error of the EGF.

Earthquake data was added to the inversion problem after iteration four. Seismic waveform data was collected from IRIS DMC for earthquakes between 1990 and 2012 with moment magnitudes $\left(M_{w}\right)$ between 5.0 and 6.0. 


\section{Synthetic Waveform Generation}

Synthetic seismograms were calculated by propagating seismic waves from a virtual source to each receiver using a nonstaggered-grid finite-difference method (Zhang et al., 2012). The initial model is a combination of the global surface wave diffraction model, CUB (Ritzwoller et al., 2002) and AK135 (Kennett et al., 1995) for depths greater than $396 \mathrm{~km}$. Wavefield simulations were carried out on a multinode Linux cluster with 24 core per node; each simulation took approximately 8 hours using a single node.

With the addition of earthquake data, inaccurate representations of the earthquake source mechanism and location needed to be addressed. As such, earthquake moment tensor solutions were gathered from the Global Centroid Moment Tensor (GCMT) catalog (Dziewonski et al., 1981). GCMT solutions were applied in finite difference forward simulations using a bell-shaped source time function (STF) of 4s duration for numerical stability purposes. To directly compare data and syn-

thetics, the synthetic waveforms were convolved with a STF scaled in duration by the earthquake magnitude. Moreover, the STF used in the finite difference simulation was convolved with the observed data. An appropriate earthquake STF was calculated based on the event's magnitude, using

$$
\begin{gathered}
L=10^{\frac{M_{w}-a}{b}} \\
T_{R}=\frac{L}{V_{R}}
\end{gathered}
$$

where $L$ is the surface rupture length, $a$ is $5.08, b$ is 1.16 from Table $2 \mathrm{~A}$ in Wells and Coppersmith (1994), $T_{R}$ and $V_{R}$ is the rupture time and velocity, $2.86 \mathrm{~km} / \mathrm{s}$.

This source equalization process is summarized in the equations below where $S_{d}$ is the observed data seismogram, $S_{s}$ is the synthetic seismogram, $G$ is the Green's 
Function, and $\Lambda_{d}$ and $\Lambda_{s}$ are the STFs of the data and synethic respectively,

$$
\begin{array}{r}
S_{d}(t)=G(t) * \Lambda_{d}(t) \\
S_{s}(t)=G(t) * \Lambda_{s}(t) \\
S_{d}^{\prime}=G(t) * \Lambda_{d}(t) * \Lambda_{s}(t) \\
S_{s}^{\prime}=G(t) * \Lambda_{s}(t) * \Lambda_{d}(t)
\end{array}
$$

The above pair of convolutions align the data and synthetics in time by accounting for phase-shifts and allowing for a straight-forward measurement to be made between the two. Filters used in the measurement are much longer in duration than either of the STFs, reducing these convolutions to simple time-shifts.

\section{Phase Delay Measurement and Inversion}

Empirical green functions (EGFs), earthquake data and synthetics were filtered, two-pass butterworth, at five overlapping finite frequency bands, $200-100 s, 150-$ $75 s, 100-50 s, 75-30 s, 50-25 s$. Phase delays, $d T$, were measured between the data and synthetics by cross-correlation for each frequency band. Low quality signals were removed from the measurements using a minimum signal-to-noise ratio and a minimum cross correlation coefficient criteria (Table 2.1). A summary of the measurement and inversion parameters are in Table 2.1. The scattering-integral (SI) approach (Zhao et al., 2005; Zhang et al., 2007; Chen et al., 2007b,a; Zhang and Shen, 2008) is used to calculate perturbations in $V_{p}$ and $V_{s}$ using a finite frequency full-waveform tomography process (see Appendix for details).

Finite frequency techniques have been used effectively to iteratively improve three-dimensional models of southern California (Tape et al., 2007, 2009), the northwestern US (Gao and Shen, 2012), Europe (Zhu et al., 2012b,a; Zhu and Tromp, 2013; Zhu et al., 2013), Tibet (Ren and Shen, 2008) and the Middle East

(Savage et al., 2012; Shen et al., 2012; Shen and Zhang, 2012). Finite frequency sensitivity kernels better recover perturbation amplitudes and wave speed geom- 
etry than ray based methods (Hung et al., 2004), reducing smearing, leading to higher amplitude and more constrained anomalies (Becker, 2012). Here we iteratively solved for absolute wave speed until changes to the 3-dimensional model were minimal using only ambient noise data. This represented four model iterations. Iterations five through our final iteration (07) included both ambient noise and earthquake data. Due to Rayleigh wave sensitivities to both shear and compressional waveforms, we solved for both absolute $V_{p}$ and $V_{s}$. Measurements were made on Rayleigh waves and our model is most sensitive to changes in $V s_{v}$ and shear wave speed structures.

\section{Results \\ Resolution}

The sparse receiver distribution in the Pacific has previously limited the ability to obtain high resolution images of the OJP region. Using our unique data set of combined ambient noise and earthquake waveform measurements, we are able to significantly improve resolution of the wave speed structure beneath the plateau from previous work (Furumoto et al., 1976; Hussong et al., 1979; Richardson et al., 2000; Gomer and Okal, 2003; Klosko et al., 2001) which either suffered from dominant north-south source/station distribution or had a geographically limited data set. To demonstrate this improved model resolution, Figure 2.2 shows the com-

putational model domain perturbed with a $5^{\circ}$ and $7^{\circ}$ sized harmonic pattern of positive and negative 5\% wave speed anomalies. For these test models the anomalies do not vary with depth. The recovered anomalies are plotted at depths of 70 , 114 and $212 \mathrm{~km}$. Resolution is excellent beneath the OJP as well as to the southeast near the Central Spreading Ridge, allowing us to confidently interpret wave speed structures down to $5^{\circ}$ in size. We outline a region in pink (Figure 2.2) that reproduces the input model well and we are confident in interpreting the recovered relative and absolute seismic wave speeds. Areas outside of the pink region are 
not well sampled. These resolution tests demonstrate that wave speed structure are best resolved between depths of 35 and $250 \mathrm{~km}$, this is similar to Gao and Shen (2014) that uses a similar methodology and period range. Interpretations of the wave speed model will be limited geographically to the pink region defined by these resolution tests.

\section{Data Fit}

Multiple metrics for assessing the robustness and model fit are used to quantify our results. Figure 2.3 shows the range of $d T$ and number of measurements at each iteration of the model. As the model evolves there is a noticeable improvement in $d T$ and reduction of error for ambient noise coupled with substantial increases in the number of measurements for both the ambient noise and earthquakes (Figure 2.3). In addition to assessment of the data set as a whole, individual measurements and event-station paths are also analyzed for evaluation. Figure 2.4 shows specific model paths for regions of interest in the model. A decrease, often significant, in the $d T$ is seen for each event-station path reflecting an improvement in the model's fit to the data. A negative $d T$ represents the need for an increase in $V s$ within the model, a positive $d T$ represents the need for a decrease in the $V s$ within the model.

\section{Seismic Wave Speed}

The resulting seismic wave speed structure is plotted in Figures 2.5 as absolute seismic shear wave speed $\left(V_{s}\right)$ in $\mathrm{km} / \mathrm{s}$. At shallow depths $(<30 \mathrm{~km}$, Figure 2.5) seismic wave speed structure is similar to Richardson et al. (2000); a broad low wave speed region in the south-southwest of the plateau corresponding to a thickened crust beneath the high plateau region. At depths deeper than the crust, however, the two models are quite different. The two most prominent and interesting features beneath the plateau are anomalously fast seismic wave speeds roughly southwest of the Nauru Basin and two small, approximately $1^{\circ}$ sized, 
anomalously slow regions to the northeast of Santa Isabel Island and beneath Guadalcanal and Malaita Islands both extending from approximately $35 \mathrm{~km}$ to greater than $100 \mathrm{~km}$.

The fast wave speed region is characterized by $V_{s}$ speeds around $4.75 \mathrm{~km} / \mathrm{s}$ with a small percentage up to $5 \mathrm{~km} / \mathrm{s}$ (see Appendix Figure 2.11); a continuously fast feature is seen extending to depths greater than $100 \mathrm{~km}$. This feature, seen in both Figure 2.5 and in cross-section (Figure 2.6), spatially corresponds with a large negative residual gravity anomaly seen in Ito and Taira (2000). Directly beneath the seismically fast region is a broad region of slow wave speeds between approximately $100-300 \mathrm{~km}$ deep.

Northeast of Santa Isabel Island is a seismically slow feature that extends roughly northwest beneath the high plateau region, seen between depths of less than $35 \mathrm{~km}$ to greater than $150 \mathrm{~km}$. Beneath Guadalcanal and Malaita a second seismically slow feature is isolated from the Santa Isabel anomaly at the surface but merges into a single feature at depth, Figure 2.6.

To the north and northwest of the observed fast structure and south of the islands of Kosrae and Pingelap, are two regions of slow seismic wave speed. From approximately $130 \mathrm{~km}$ to greater than $300 \mathrm{~km}$, fast seismic wave speeds transition to a broad region of slow wave speeds beneath the plateau.

The main feature spans from $-1^{\circ}$ to $7^{\circ}$ in latitude and is distinct and isolated from the skinnier more column-like feature under the Solomon Islands. The seismically slow features of Kosrae, Pingelap and Santa Isabel roughly surround the main fast wave speed anomaly in three dimensions.

Southeast of the plateau and roughly aligned with the Vitiaz lineament is a distinct boundary between old, seismically fast Pacific seafloor to the north and much younger, seismically slow seafloor of the Balmoral Reef and New Hebrides microplates. Slow seismic wave speeds are attributed to active seafloor spreading at 
ridges adjacent to the New Hebrides, Balmoral Reef, Conway Reef and Australian plates near the Fiji platform. Slow regions associated with the Fiji and Samoan plumes can be seen extending to depths greater than $350 \mathrm{~km}$ in Figure 2.6, though our resolution is limited below approximately $250 \mathrm{~km}$.

West of the OJP, slow seismic wave speeds trace the boundary between the North Bismark/Manus plates and the South Bismark plate northeast of Papua New Guinea and extend in a column-like structure to depths greater than $150 \mathrm{~km}$. A region of fast seismic wave speeds neatly outlines the Solomon Sea plate which can be seen subducting to the north beneath the South Bismark plate, Figure 2.6.

To assess whether the methodology and data distribution are able to resolve such a large-scale, fast wave speed anomaly beneath the plateau we constructed a synthetic model with a single isolated wave speed perturbation in the location of our observed anomaly. This synthetic model demonstrates how the anomaly is smeared and reduced in absolute amplitude by the inversion method. Figure 2.7 shows that we are able to recover the amplitude of the input perturbation well and with limited smearing to regions adjacent to the anomaly for a single iteration.

In addition, the ability to resolve other known seismic structures within our model space demonstrates the resolving power and robustness of our observations and methodology. For example, the boundary in the vicinity of the Vitiaz lineament between $120+$ Ma, seismically fast Pacific seafloor to the north and $<10 \mathrm{Ma}$, seismically slow seafloor to the south (Müller et al., 2008)provides a well-defined boundary to test our resolving ability of distinct, short-wavelength, wave speed boundaries. Figure 2.7 shows the wave speed model near the Vitiaz lineament with limited smearing across the boundary. In addition, slow wave speeds corresponding to centers of recent volcanism on the islands of Santa Isabel, Guadalcanal and Malaita (Tejada et al., 1996) suggest that we are possibly resolving the source of this recent volcanism. To the west of the OJP, slow wave speeds roughly trace the 
plate boundary between the North Bismark/Manus and the South Bismark plates (Bird, 2003). The dense, subducting Solomon Sea plate is also neatly outlined by a seismically fast region in our model (between approximately $150^{\circ}-154^{\circ}$ ). The agreement of these features in our model with known geologic structures further provides confidence in our model's resolving capabilities.

\section{Discussion}

Our resulting model has a region of fast shear wave speeds beneath the OJP that differs significantly from previous Rayleigh wave tomography by Richardson et al. (2000) and is faster than 120 Ma oceanic lithosphere (Maggi et al., 2006). We can rule out melt and/or volatiles as a source of the fast anomaly, as their presence would decrease shear wave speed (Karato and Jung, 1998; Hammond and Humphreys, 2000). In the following, we discuss three possible explanations, 1) the observed data do not require such high wave speeds, 2) there is significant $V s_{v}>V s_{h}$ anisotropy in the region or 3) the wave speed structure is a result of a mantle compositionally different than 120 Ma oceanic mantle.

\section{Are the very high wave speeds required by the observed data?}

To address question 1 we forward simulate select event-station pairs that cross the high wave speed anomaly using two model cases, limiting $V_{s}$ values to a maximum of a) 4.5 and b) $4.75 \mathrm{~km} / \mathrm{s}$ above $400 \mathrm{~km}$ depth. Shown in Figure 2.8 is our calculated waveforms from the final iteration model plotted on top of the data and the results of the capped models. There is a significant degradation in fit when using a $4.5 \mathrm{~km} / \mathrm{s}$ capped model. The main surface wave arrival in the $4.5 \mathrm{~km} / \mathrm{s}$ capped synthetic arrives on average 17 seconds later than the data; compared to the synthetic from our final iteration model, which matches the data's phase quite well. A $4.75 \mathrm{~km} / \mathrm{s}$ capped model fits more closely with our final iteration results and the data. At the longest periods, $>75$ seconds, the $4.75 \mathrm{~km} / \mathrm{s}$ capped model is nearly identical to our final iteration results. At shorter periods, $25-50 \mathrm{~s}$, small 
differences in the main surface wave arrival are present. Based on the above capping tests, we can confidently identify a wave speed $>4.75 \mathrm{~km} / \mathrm{s}$ in the fastest portion of the model beneath the OJP. These wave speed represent absolute values similar to cratonic lithosphere (Grand and Helmberger, 1984; Kustowski et al., 2008; Dalton et al., 2009; Panning et al., 2010; Ritsema et al., 2011) and faster than the expected shear wave speeds of 120 Ma oceanic lithosphere (Maggi et al., 2006; Beghein et al., 2014).

\section{Anisotropy}

The second possibility for the anomalously fast wave speeds could be related to $V s_{h} / V s_{v}$ anisotropy. To test this assumption, we compare our isotropic synthetic transverse component wavefield, $V s_{h}=V s_{v}$, to the transverse component of the data and two separate anisotropic cases for three event-station pairs that sample the anomaly, 1) 1999/12/08 13:34:42 II.KWAJ, 2) 2000/08/14 22:11:16 II.KWAJ and 3) 2007/04/02 10:49:19 PS.PATS.

In case 1 we reduce our $V s$ values by the percentage of $V s_{h} / V s_{v}$ anisotropy in anisotropic PREM (Dziewonski et al., 1981), a max of $1 \%$ at the very top of the mantle. This is analogous to an observed anisotropy where $V s_{v}$ is the fast polarization direction and $V s_{h}$ represents the slow axis. This has been observed by Beghein et al. (2014) in the top $80 \mathrm{~km}$ of $80-120$ Ma oceanic lithosphere. The results, shown in Figure 2.9, reveal a transverse component arrival that is late compared to the data. This eliminates the possibility that the fast wave speeds measured on the vertical component are a result of $V s_{v}>V s_{h}$ anisotropy.

In case 2 we increase our $V s$ values by the percentage of $V s_{h} / V s_{v}$ anisotropy in anisotropic PREM. This is analogous to adding PREM anisotropy to our calculated values, $V s_{h}>V s_{v}$. For all three event-station pairs, the anisotropic case fits the data better than the purely isotropic model, seen as a decrease in the $d T$ for nearly

all frequency bands (Figure 2.9). The ability to fit both vertical and transverse 
component surface waves when applying simple $V s_{h}>V s_{v}$ anisotropy, suggests that the fast anomaly is probably anisotropic.

\section{Composition}

Finally we are left with a compositional source for the fast wave speeds in the region. Historically, eclogite has been used to account for such high wave speeds in other anomalous regions throughout the world. Ultra high pressure (UHP) eclogites from Sulu and Dabie region in China are weakly anisotropic $(<3 \%)$ and have fast axis $V s$ and slow axis $V s$ velocities ranging from $4.89-5.05 \mathrm{~km} / \mathrm{s}$ and $4.84-5.01 \mathrm{~km} / \mathrm{s}$ respectively (Bascou et al., 2001; Ji et al., 2003; Zhang et al., 2008) . Eclogite samples from the Western Gneiss Region in Norway show Vs wave speeds ranging from $4.5-4.99 \mathrm{~km} / \mathrm{s}$ (Bascou et al., 2001; Worthington et al., 2013). Several studies of the Slave Craton in northern Canada have shear wave speeds similarly fast as our results for OJP (Cammarano and Romanowicz, 2007; Romanowicz, 2009; Fischer et al., 2010) and eclogite has been interpreted as the source for these abnormally fast wave speeds (Snyder, 2008).

To discriminate between a garnet-rich fertile peridotite and various types of eclogite, we ran sample cases using the Excel macro of Hacker and Abers (2004). We added garnet to a fertile peridotite with modal abundances of $55 \%$ forsterite, $25 \%$ enstatite, $18 \%$ diopside. Garnet was added in increments of 2\%, 4\%,6\%, $8 \%, 10 \%$ and $20 \%$ as the modal abundances of the other minerals was adjusted proportionally, see Table 2.2 for modal abundances. Mineral wave speeds were simulated at $2.5 \mathrm{GPa}$ and a lithosphere temperature of $1125^{\circ} \mathrm{C}$ at that depth, which is an approximate geotherm of $15^{\circ} \mathrm{C}$ per $\mathrm{km}$ (Stein and Stein, 1992). An $1125^{\circ} \mathrm{C}$ estimated lithosphere temperature falls within the range of possible values for 120 Ma lithosphere at $70 \mathrm{~km}$ (Stein and Stein, 1992). Calculated shear wave speed is less than $4.65 \mathrm{~km} / \mathrm{s}$ for all garnet-rich peridotite cases (Table 2.2) . We then calculated a suite of eclogite compositions, again incrementally increasing the 
$\%$ of garnet $(20 \%, 30 \%, 40 \%, 50 \%, 60 \%)$. Modal abundances of zoisite amphibole eclogite, amphibole eclogite, zoisite eclogite, lawsonite amphibole eclogite, coesite eclogite, and diamond eclogite were also used (Hacker and Abers, 2004). Calculated shear wave speed was less than $4.50 \mathrm{~km} / \mathrm{s}$ for all eclogite cases. Finally wave speeds were calculated using approximate modal abundances for UHP eclogites from Ji et al. (2003) that contain large amounts of garnet and jadeite, see Table 2.2 for compositions. We were able to reproduce the measurements of $V_{s}$ to those measured in the literature (Bascou et al., 2001; Ji et al., 2003; Zhang et al., 2008) for UHP eclogite compositions.

Our resulting wave speeds and the above sensitivity tests support a possible compositional anomaly for the fast wave speeds in the region. This does not discriminate, however, between the potential plateau formation theories, as both a vigorous upwelling (Korenaga, 2005) or a plume scenario (Bercovici and Mahoney, 1994; Larson and Kincaid, 1996; Tejada et al., 1996; Jain et al., 1996; Neal et al., 1997; Michael, 1999; Ely and Neal, 2003; Ishikawa et al., 2004, 2007) could support entrainment of eclogite. Korenaga (2005) suggested the entrainment of dense eclogite fragments non-uniformally distributed throughout the mantle to explain the intriguing buoyancy characteristics of the plateau. Ishikawa et al. $(2004,2007)$ showed evidence for significant amounts of ancient recycled subducted crust in their xenolith samples from mantle beneath the OJP. The authors suggest a chemically heterogeneous plume (Ishikawa et al., 2004, 2007) as the source of the OJP. Models of thermochemical plume heads, with as much as $15 w t \%$ eclogite, have also been shown to retard surface uplift, as well as cause extensive delamination and thinning of the lithosphere, compared to a purely thermal plume head case (Sobolev et al., 2011). Further, Silver et al. (2006) suggested the presence of excess heat at the base of the lithosphere representing remanent portions of plumes unable to penetrate a cratonic lithosphere. This hypothesis could explain the slow 
wave speeds seen directly beneath the shallower seismically fast and likely strong anomaly on the plateau. An eclogite composition could also reconcile the anomalous subsidence history of the plateau. Foundering or delamination of the eclogite could allow the plateau to remain relatively buoyant over the last 120 Ma.

In addition to the plume or vigorous upwelling hypotheses, Ito and Taira (2000) suggest two models to explain a Bouguer anomaly greater than predicted Airy isostasy beneath the plateau. The first involved late stage magmatic underplating beneath an already thickened plate. It is possible that this underplating could force oceanic crust within the stability range for eclogite formation.

Additional evidence from geochemistry is needed to further discriminate between source hypotheses. Geochemically, samples represent high degrees of melting to a well-buffered OIB-like source (Tejada et al., 1996; Michael, 1999). Enrichment in siderophile elements, such as molybdenum (Mo), members of the platinum group, and gold $(\mathrm{Au})$, suggest a core-mantle boundary origin that is consistent with a plume hypothesis (Jain et al., 1996; Neal et al., 1997; Ely and Neal, 2003).

A compositionally heterogeneous mantle beneath the OJP is required to generate the observed wave speed structure seen in our model. Regions of fast wave speed, $>4.75 \mathrm{~km} / \mathrm{s}$, can be explained with a garnet rich composition that is likely eclogite. The fast seismic wave speeds suggest a feature that is strong, able to persist $120 \mathrm{Ma}$ and is related to the plateau's formation. The feature may represents remnants of a larger structure that has undergone erosion due to $120 \mathrm{Ma}$ of plate motion. Laboratory tank modeling suggests that the plate motion history is extremely important to the interpretation of the modern tectonic features (Druken et al., 2013; Kincaid et al., 2013; Druken et al., 2014) . Mantle fabrics present $120 \mathrm{Ma}$ ago are likely to be significantly deformed as a result of complex interactions with plate tectonic process. While our seismic model can not discriminate between the two main plateau formation hypotheses, the fast wave speeds in 
combination with previous geochemical observations is consistent with hypotheses for a compositionally heterogeneous plume with entrained eclogite.

\section{Conclusions}

A unique dataset using a combination of ambient noise and earthquake waveforms was used to determine the seismic wave speed structure of the Ontong Java Plateau. Our model's resolution represents a significant improvement over previous research and the highest wave speed resolution to date for the region. We have shown a significant improvement in our model relative to the starting model, CUB (Ritzwoller et al., 2002), seen as a decrease in the phase delay, $d T$, through iterations, Figure 2.3, and in the synthetic waveform fit to the data, Figure 2.4.

Beneath the plateau we image a region of shear wave speeds $>4.75 \mathrm{~km} / \mathrm{s}$, possibly up to $5.00 \mathrm{~km} / \mathrm{s}$. These wave speeds are faster than normal oceanic lithosphere $120 \mathrm{Ma}$ in age (approximately $4.5 \mathrm{~km} / \mathrm{s}$ ) (Maggi et al., 2006; Beghein et al., 2014) and are similar to as observed in cratonic environments (Grand and Helmberger, 1984; Kustowski et al., 2008; Dalton et al., 2009; Panning et al., 2010; Ritsema et al., 2011). Tests for $V s_{v}>V s_{h}$ anisotropy through the fast anomaly are ruled out by the data. However, the addition of simple $V s_{h}>V s_{v}$ anisotropy slightly improves synthetic fit to the data relative to our isotropic model.

Our observed wave speeds beneath the plateau are consistent with a compositional anomaly and likely a result of UHP eclogite compositions. While our seismic model cannot conclusively discriminate between the two major formation hypotheses, previous studies of rock samples from the Solomon Islands suggest a compositionally heterogenous plume source for the OJP (Tejada et al., 1996; Jain et al., 1996; Neal et al., 1997; Michael, 1999; Ely and Neal, 2003). We suggest that the surfacing plume head entrained eclogite from the deep mantle, resulting in a denser than normal eruption that retarded surface uplift and accounts for the 
observed fast wave speeds beneath the plateau.

\section{Acknowledgments}

We would like to thank Rob Pockalny, Chris Kincaid, Catherine Rychert, Nick

Harmon, Karen Fischer and Paul Hall for their helpful comments, valuable insights and opinions during our interpretation. We would also like to thank Maryjo Brounce for her help with geochemical calculations and using Excel. 


\section{BIBLIOGRAPHY}

Bascou, J., G. Barruol, A. Vauchez, D. Mainprice, and M. Egydio-Silva, Ebsdmeasured lattice-preferred orientations and seismic properties of eclogites, Tectonophysics, 342(1), 61-80, 2001.

Becker, T., On recent seismic tomography for the western united states, Geochemistry Geophysics Geosystems, 13, 2012.

Beghein, C., K. Yuan, N. Schmerr, and Z. Xing, Changes in seismic anisotropy shed light on the nature of the gutenberg discontinuity., Science (New York, NY), 2014.

Bercovici, D., and J. Mahoney, Double flood basalts and plume head separation at the 660-kilometer discontinuity, Science, 266 (5189), 1367-1369, 1994.

Billen, M. I., and J. Stock, Morphology and origin of the osbourn trough, Journal of Geophysical Research: Solid Earth (1978-2012), 105(B6), 13,481-13,489, 2000.

Bird, P., An updated digital model of plate boundaries, Geochemistry, Geophysics, Geosystems, 4(3), 2003.

Cammarano, F., and B. Romanowicz, Insights into the nature of the transition zone from physically constrained inversion of long-period seismic data, Proceedings of the National Academy of Sciences, 104(22), 9139-9144, 2007.

Campbell, I., The Earth's Mantle: Composition, Structure and Evolution, chap. The mantle's chemical structure: insights from the melting products of mantle plumes, pp. 259-310, 1998.

Campbell, I. H., Large igneous provinces and the mantle plume hypothesis, Elements, 1(5), 265-269, 2005. 
Chandler, M. T., P. Wessel, B. Taylor, M. Seton, S.-S. Kim, and K. Hyeong, Reconstructing ontong java nui: Implications for pacific absolute plate motion, hotspot drift and true polar wander, Earth and Planetary Science Letters, 331, $140-151,2012$.

Chen, P., T. H. Jordan, and L. Zhao, Full three-dimensional tomography: a comparison between the scattering-integral and adjoint-wavefield methods, Geophysical Journal International, 170(1), 175-181, 2007a.

Chen, P., L. Zhao, and T. H. Jordan, Full 3d tomography for the crustal structure of the los angeles region, Bulletin of the Seismological Society of America, 97(4), 1094-1120, 2007b.

Coffin, M., and O. Eldholm, Large igneous provinces: crustal structure, dimensions, and external consequences, Reviews of Geophysics, 32, 1-1, 1994.

Crisp, J. A., Rates of magma emplacement and volcanic output, Journal of Volcanology and Geothermal Research, 20(3), 177-211, 1984.

Dalton, C. A., G. Ekström, and A. M. Dziewonski, Global seismological shear velocity and attenuation: A comparison with experimental observations, Earth and Planetary Science Letters, 284(1), 65-75, 2009.

Druken, K., C. Kincaid, and R. Griffiths, Directions of seismic anisotropy in laboratory models of mantle plumes, Geophysical Research Letters, 40(14), 3544-3549, 2013.

Druken, K., C. Kincaid, R. Griffiths, D. Stegman, and S. Hart, Plume-slab interaction: The samoa-tonga system, Physics of the Earth and Planetary Interiors, 2014. 
Dziewonski, A., T. Chou, and J. Woodhouse, Determination of earthquake source parameters from waveform data for studies of global and regional seismicity, Journal of Geophysical Research, 86(B4), 2825-2852, 1981.

Ely, J., and C. Neal, Using platinum-group elements to investigate the origin of the ontong java plateau, sw pacific, Chemical Geology, 196(1-4), 235-257, 2003.

Farnetani, C., and M. Richards, Numerical investigations of the mantle plume initiation model for flood basalt events, Journal of Geophysical Research, 99, 13-13, 1994.

Fischer, K. M., H. A. Ford, D. L. Abt, and C. A. Rychert, The lithosphereasthenosphere boundary, Annual Review of Earth and Planetary Sciences, 38, $551-575,2010$.

Furumoto, A. S., J. P. Webb, M. E. Odegard, and D. M. Hussong, Seismic studies on the ontong java plateau, 1970, Tectonophysics, 34(1), 71-90, 1976.

Gao, H., and Y. Shen, Validation of shear-wave velocity models of the pacific northwest, Bulletin of the Seismological Society of America, 102(6), 2611-2621, 2012.

Gao, H., and Y. Shen, Upper mantle structure of the cascades from full-wave ambient noise tomography: Evidence for 3d mantle upwelling in the back-arc, Earth and Planetary Science Letters, 390, 222-233, 2014.

Gee, L. S., and T. H. Jordan, Generalized seismological data functionals, Geophysical Journal International, 111(2), 363-390, 1992.

Gladczenko, T., M. Coffin, and O. Eldholm, Crustal structure of the ontong java plateau: modeling of new gravity and existing seismic data, Journal of Geophysical Research, 102, 22-22, 1997. 
Gomer, B., and E. Okal, Multiple-scs probing of the ontong-java plateau, Physics of the Earth and Planetary Interiors, 138(3), 317-331, 2003.

Grand, S. P., and D. V. Helmberger, Upper mantle shear structure of north america, Geophysical Journal International, 76(2), 399-438, 1984.

Griffiths, R., M. Gurnis, and G. Eitelberg, Holographic measurements of surface topography in laboratory models of mantle hotspots, Geophysical Journal International, 96(3), 477-495, 1989.

Hacker, B. R., and G. A. Abers, Subduction factory 3: An excel worksheet and macro for calculating the densities, seismic wave speeds, and h2o contents of minerals and rocks at pressure and temperature, Geochemistry, Geophysics, Geosystems, 5(1), 2004.

Hall, P. S., and C. Kincaid, Melting, dehydration, and the geochemistry of off-axis plume-ridge interaction, Geochemistry, Geophysics, Geosystems, 5(12), 2004.

Hammond, W., and E. Humphreys, Upper mantle seismic wave velocity: Effects of realistic partial melt geometries, Jour. Geophys. Res., 105(B5), 2000.

Hung, S., Y. Shen, and L. Chiao, Imaging seismic velocity structure beneath the iceland hot spot: A finite frequency approach, Journal of geophysical research, $109(\mathrm{~B} 8)$, B08,305, 2004.

Hussong, D., L. Wipperman, and L. Kroenke, The crustal structure of the ontong java and manihiki oceanic plateaus, Journal of Geophysical Research, 84(B11), 6003-6010, 1979.

Ishikawa, A., S. Maruyama, and T. Komiya, Layered lithospheric mantle beneath the ontong java plateau: implications from xenoliths in alnöite, malaita, solomon islands, Journal of Petrology, 45(10), 2011-2044, 2004. 
Ishikawa, A., T. Kuritani, A. Makishima, and E. Nakamura, Ancient recycled crust beneath the ontong java plateau: Isotopic evidence from the garnet clinopyroxenite xenoliths, malaita, solomon islands, Earth and Planetary Science Letters, $259(1), 134-148,2007$.

Ito, G., and A. Taira, Compensation of the ontong java plateau by surface and subsurface loading, Journal of geophysical research, 105(11), 171-11, 2000.

Ito, G., and P. van Keken, Hotspots and melting anomalies, Treatise on Geophysics, 7, 371-436, 2007.

Jain, J., C. Neal, and J. O’Neill Jr, Origin of the ontong java plateau (ojp) at the core-mantle boundary: platinum group element (pge) and gold (au) evidence, Transactions of the American Geophysical Union, EOS, 77, 714, 1996.

Ji, S., K. Saruwatari, D. Mainprice, R. Wirth, Z. Xu, and B. Xia, Microstructures, petrofabrics and seismic properties of ultra high-pressure eclogites from sulu region, china: implications for rheology of subducted continental crust and origin of mantle reflections, Tectonophysics, 370(1), 49-76, 2003.

Karato, S., and H. Jung, Water, partial melting and the origin of the seismic low velocity and high attenuation zone in the upper mantle, Earth and Planetary Science Letters, 157(3-4), 193-207, 1998.

Kennett, B., E. Engdahl, and R. Buland, Constraints on seismic velocities in the earth from traveltimes, Geophysical Journal International, 122(1), 108-124, 1995.

Kincaid, C., K. Druken, R. Griffiths, and D. Stegman, Bifurcation of the yellowstone plume driven by subduction-induced mantle flow, Nature Geoscience, 6(5), 395-399, 2013. 
Klosko, E., R. Russo, E. Okal, and W. Richardson, Evidence for a rheologically strong chemical mantle root beneath the ontong-java plateau, Earth and Planetary Science Letters, 186(3), 347-361, 2001.

Korenaga, J., Why did not the ontong java plateau form subaerially?, Earth and Planetary Science Letters, 234(3-4), 385-399, 2005.

Kustowski, B., G. Ekström, and A. Dziewoński, Anisotropic shear-wave velocity structure of the earth's mantle: A global model, Journal of Geophysical Research: Solid Earth (1978-2012), 113(B6), 2008.

Larson, R., Latest pulse of earth: Evidence for a mid-cretaceous superplume, $G e$ ology, 19(6), 547-550, 1991.

Larson, R., Superplumes and ridge interactions between ontong java and manihiki plateaus and the nova-canton trough, Geology, 25(9), 779-782, 1997.

Larson, R. L., and C. Kincaid, Onset of mid-cretaceous volcanism by elevation of the $670 \mathrm{~km}$ thermal boundary layer, Geology, 24(6), 551-554, 1996.

Maggi, A., E. Debayle, K. Priestley, and G. Barruol, Multimode surface waveform tomography of the pacific ocean: a closer look at the lithospheric cooling signature, Geophysical Journal International, 166(3), 1384-1397, 2006.

Mahoney, J., J. Fitton, and P. Wallace, Initial reports, in Proceedings of the Ocean Drilling Program, vol. 192, 2001.

Michael, P., Implications for magmatic processes at ontong java plateau from volatile and major element contents of cretaceous basalt glasses, Geochemistry Geophysics Geosystems, 1(12), 1008-17, 1999.

Müller, R. D., M. Sdrolias, C. Gaina, and W. R. Roest, Age, spreading rates, 
and spreading asymmetry of the world's ocean crust, Geochemistry, Geophysics, Geosystems, 9(4), 2008.

Nakanishi, M., K. Tamaki, and K. Kobayashi, Magnetic anomaly lineations from late jurassic to early cretaceous in the west-central pacific ocean, Geophysical Journal International, $109(3)$, 701-719, 1992.

Neal, C., J. Mahoney, L. Kroenke, R. Duncan, and M. Petterson, Large Igneous Provinces: Continental, Oceanic, and Planetary Flood Volcanism, vol. 100, chap. The Ontong Java Plateau, pp. 183-216, 1997.

Olson, P., Hot spots, swells and mantle plumes, Magma transport and storage, pp. $33-51,1990$.

Panning, M., V. Lekić, and B. Romanowicz, Importance of crustal corrections in the development of a new global model of radial anisotropy, Journal of Geophysical Research: Solid Earth (1978-2012), 115(B12), 2010.

Ren, Y., and Y. Shen, Finite frequency tomography in southeastern tibet: evidence for the causal relationship between mantle lithosphere delamination and the north-south trending rifts, Journal of Geophysical Research: Solid Earth (19782012), $113(\mathrm{~B} 10), 2008$.

Richardson, W., E. Okal, and S. Van der Lee, Rayleigh-wave tomography of the ontong-java plateau, Physics of the Earth and Planetary Interiors, 118(1-2), $29-51,2000$.

Ritsema, J., A. Deuss, H. Van Heijst, and J. Woodhouse, S40rts: a degree-40 shear-velocity model for the mantle from new rayleigh wave dispersion, teleseismic traveltime and normal-mode splitting function measurements, Geophysical Journal International, 184(3), 1223-1236, 2011. 
Ritzwoller, M. H., N. M. Shapiro, M. P. Barmin, and A. L. Levshin, Global surface wave diffraction tomography, Journal of Geophysical Research: Solid Earth (1978-2012), 107(B12), ESE-4, 2002.

Romanowicz, B., The thickness of tectonic plates, Science, 324(5926), 474-476, 2009.

Savage, B., D. Peter, B. M. Covellone, C. Morency, A. Rodgers, and J. Tromp, Short-period, anelastic, and anisotropic, waveform-based 3d middle east model to improve nuclear explosion monitoring, in Proceedings: 34th Monitoring Research Review (MRR 2012), 2012.

Shen, Y., and W. Zhang, Multi-grid and resolution full-wave tomography and moment tensor inversion, in Proceedings: 34th Monitoring Research Review (MRR 2012), 2012.

Shen, Y., Y. Ren, H. Gao, and B. Savage, An improved method to extract verybroadband empirical green's functions from ambient seismic noise, Bulletin of the Seismological Society of America, 102(4), 1872-1877, 2012.

Silver, P. G., M. D. Behn, K. Kelley, M. Schmitz, and B. Savage, Understanding cratonic flood basalts, Earth and Planetary Science Letters, 245(1), 190-201, 2006.

Snyder, D. B., Stacked uppermost mantle layers within the slave craton of nw canada as defined by anisotropic seismic discontinuities, Tectonics, 27(4), 2008.

Sobolev, S., A. Sobolev, D. Kuzmin, N. Krivolutskaya, A. Petrunin, N. Arndt, V. Radko, and Y. Vasiliev, Linking mantle plumes, large igneous provinces and environmental catastrophes, Nature, 477(7364), 312-316, 2011. 
Stein, C. A., and S. Stein, A model for the global variation in oceanic depth and heat flow with lithospheric age, Nature, 359(6391), 123-129, 1992.

Tape, C., Q. Liu, and J. Tromp, Finite-frequency tomography using adjoint methods - methodology and examples using membrane surface waves, Geophysical Journal International, 168(3), 1105-1129, 2007.

Tape, C., Q. Liu, A. Maggi, and J. Tromp, Adjoint tomography of the southern California crust, Science, 325(5943), 988, 2009.

Taylor, B., The single largest oceanic plateau: Ontong java-manihiki-hikurangi, Earth and Planetary Science Letters, 241(3-4), 372-380, 2006.

Tejada, M., J. Mahoney, R. Duncan, and M. Hawkins, Age and geochemistry of basement and alkalic rocks of malaita and santa isabel, solomon islands, southern margin of ontong java plateau, Journal of Petrology, 37(2), 361-394, 1996.

Tejada, M., J. Mahoney, C. Neal, R. Duncan, and M. Petterson, Basement geochemistry and geochronology of central malaita, solomon islands, with implications for the origin and evolution of the ontong java plateau, Journal of Petrology, 43(3), 449-484, 2002.

Tejada, M., J. Mahoney, P. Castillo, S. Ingle, H. Sheth, and D. Weis, Pin-pricking the elephant: Evidence on the origin of the ontong java plateau from pb-sr-hfnd isotopic characteristics of odp leg 192 basalts, Geological Society, London, Special Publications, 229(1), 133-150, 2004.

Viso, R. F., R. L. Larson, and R. A. Pockalny, Tectonic evolution of the pacificphoenix-farallon triple junction in the south pacific ocean, Earth and Planetary Science Letters, 233(1), 179-194, 2005. 
Wells, D. L., and K. J. Coppersmith, New empirical relationships among magnitude, rupture length, rupture width, rupture area, and surface displacement, Bulletin of the Seismological Society of America, 84(4), 974-1002, 1994.

Worthington, J. R., B. R. Hacker, and G. Zandt, Distinguishing eclogite from peridotite: Ebsd-based calculations of seismic velocities, Geophysical Journal International, 193(1), 489-505, 2013.

Zhang, J., Y. Wang, and Z. Jin, Cpo-induced seismic anisotropy in uhp eclogites, Science in China Series D: Earth Sciences, 51(1), 11-21, 2008.

Zhang, W., Y. Shen, and L. Zhao, Three-dimensional anisotropic seismic wave modelling in spherical coordinates by a collocated-grid finite-difference method, Geophysical Journal International, 188(3), 1359-1381, 2012.

Zhang, Z., and Y. Shen, Cross-dependence of finite-frequency compressional waveforms to shear seismic wave speeds, Geophysical Journal International, 174(3), 941-948, 2008.

Zhang, Z., Y. Shen, and L. Zhao, Finite-frequency sensitivity kernels for head waves, Geophysical Journal International, 171(2), 847-856, 2007.

Zhao, L., T. Jordan, K. Olsen, and P. Chen, Fréchet kernels for imaging regional earth structure based on three-dimensional reference models, Bulletin of the Seismological Society of America, 95(6), 2066, 2005.

Zhu, H., and J. Tromp, Mapping tectonic deformation in the crust and upper mantle beneath europe and the north atlantic ocean, Science, 341(6148), 871875, 2013.

Zhu, H., E. Bozdăg, D. Peter, and J. Tromp, Seismic wavespeed images across the iapetus and tornquist suture zones, Geophysical Research Letters, 39(18), 2012a. 
Zhu, H., E. Bozdağ, D. Peter, and J. Tromp, Structure of the european upper mantle revealed by adjoint tomography, Nature Geoscience, 5(7), 493-498, 2012b.

Zhu, H., E. Bozdăg, T. S. Duffy, and J. Tromp, Seismic attenuation beneath europe and the north atlantic: Implications for water in the mantle, Earth and Planetary Science Letters, 381, 1-11, 2013. 


\begin{tabular}{|c|c|c|c|c|}
\hline Iteration & Data Type & $\begin{array}{c}\text { dT Threshold } \\
\text { (seconds) }\end{array}$ & Damping & Smoothing \\
\hline \hline 1 & AN & 35 & 16 & 16 \\
2 & AN & 35 & 4 & 16 \\
3 & AN & 35 & 4 & 12 \\
4 & AN & 35 & 4 & 8 \\
5 & AN and EQ & 35 & 25 & 16 \\
6 & AN and EQ & 35 & 25 & 10 \\
7 & AN and EQ & 35 & 25 & 10 \\
\hline
\end{tabular}

Table 2.1: Summary of inversion parameters.

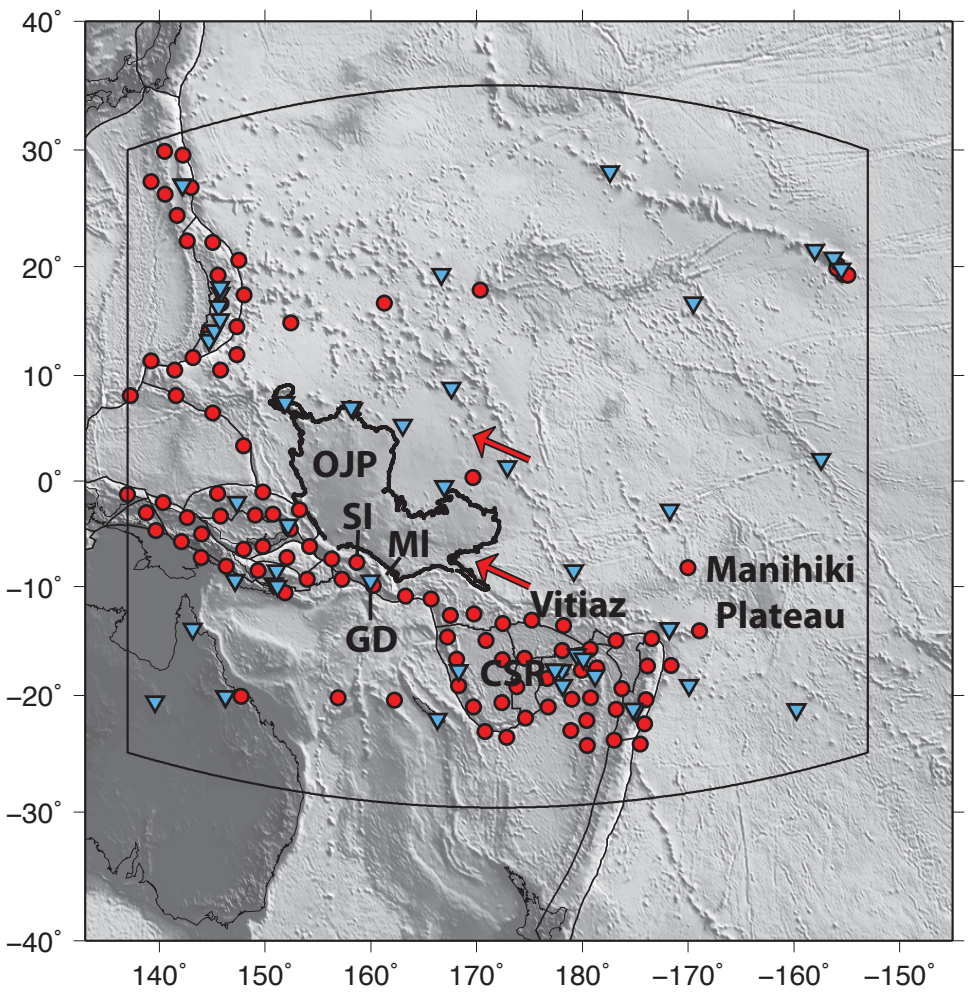

Figure 2.1: 54 Seismic stations (inverted triangles) and 105 earthquake events (circles) used for this study in the Pacific Ocean. Our computational domain is outlined in black. The $4000 \mathrm{~m}$ bathymetric contour of the Ontong Java Plateau (OJP) is outlined in black. Modern plate boundaries are shown with a thin black line. The red arrows show modern Pacific plate motion. Major features have been labeled in black: Central Spreading Ridge (CSR), the Vitiaz Lineament, and Manihiki Plateau. The location of Solomon Islands of Santa Isabel Island (SI), Guadalcanal (GD), and Malaita Island (MI) are also highlighted. 

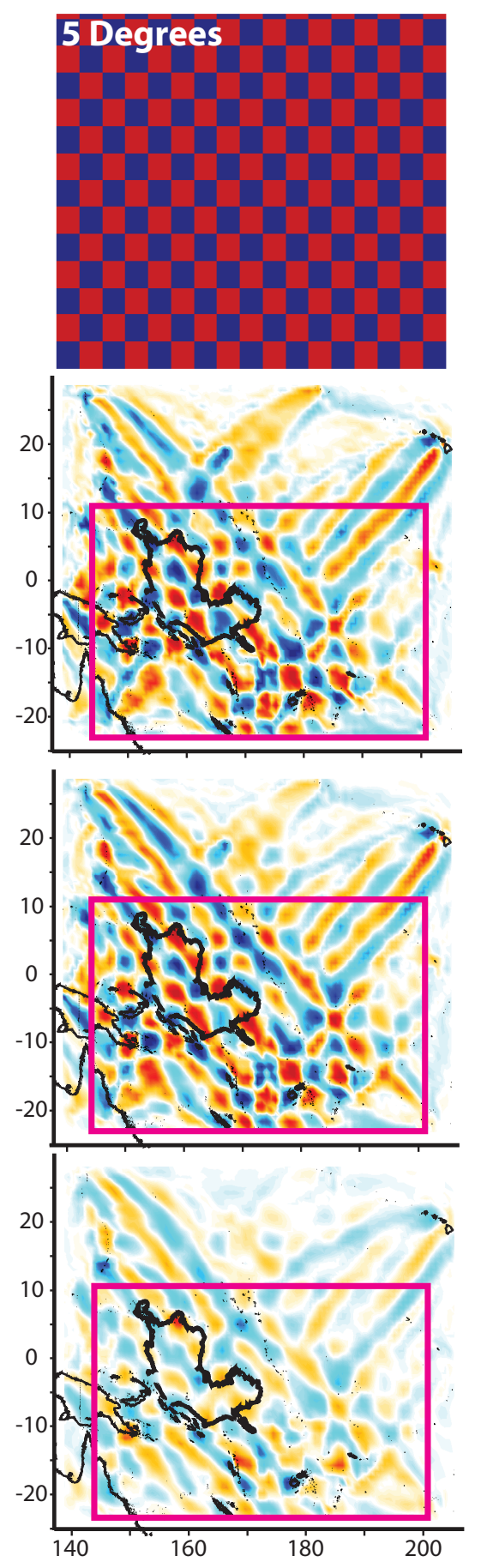
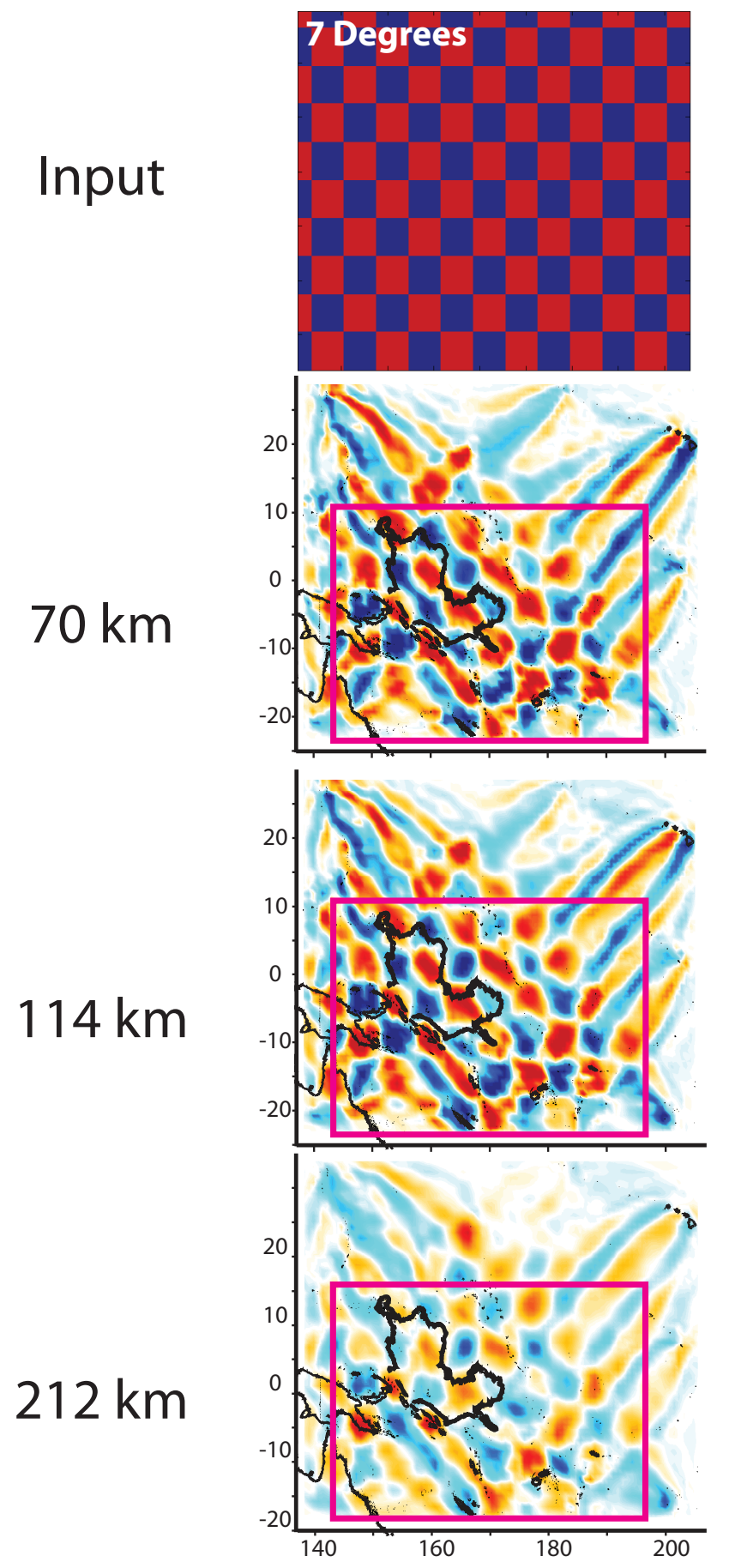

Figure 2.2: Computational model domain perturbed with $5^{\circ}$ and $7^{\circ}$ sized harmonic positive and negative $5 \%$ wave speed anomalies. Our recovered solution is plotted at three depths into the model. Our recovered resolution is excellent for the region outlined in pink. 

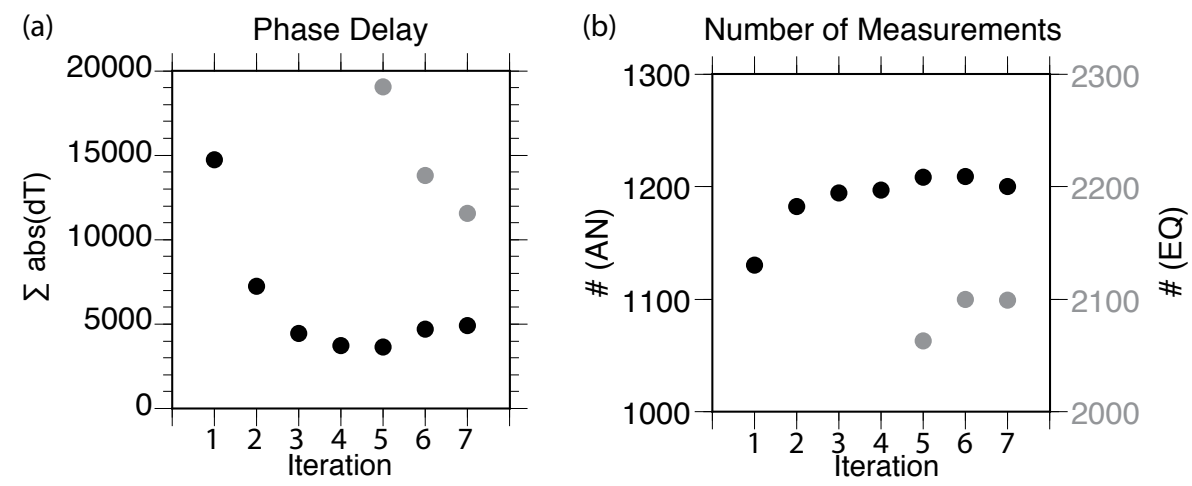

Figure 2.3: (a) Shows the evolution of the $d T$ through iterations; black circles are from AN data, grey circles are from EQ data. The sum of the absolute value of the $d T$ provides a good indication of how the whole data set is improving despite the increase in the number of measurements shown in (b). An increase in the number of measurements through iteration reflects an improvement in fit to the data. 


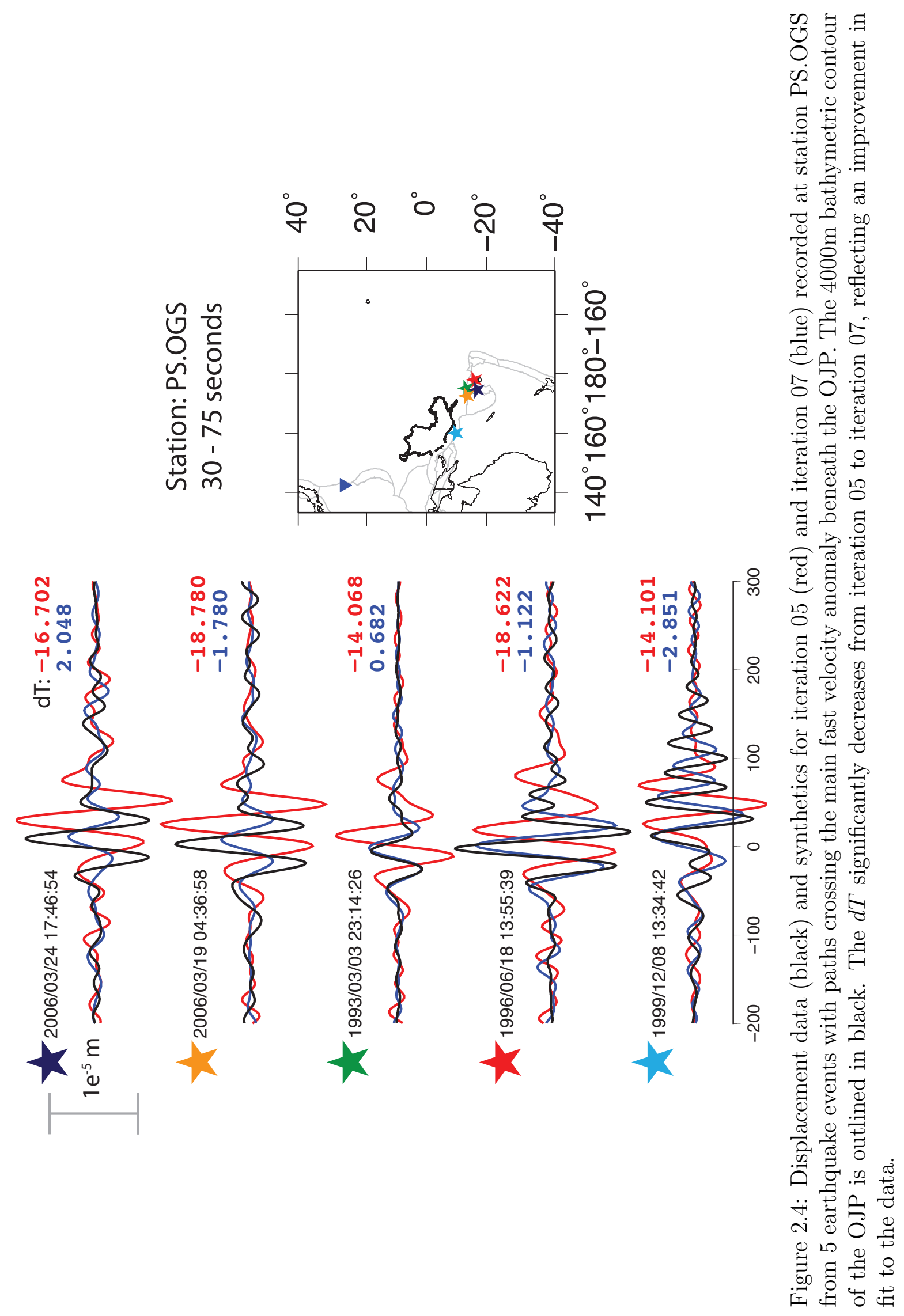



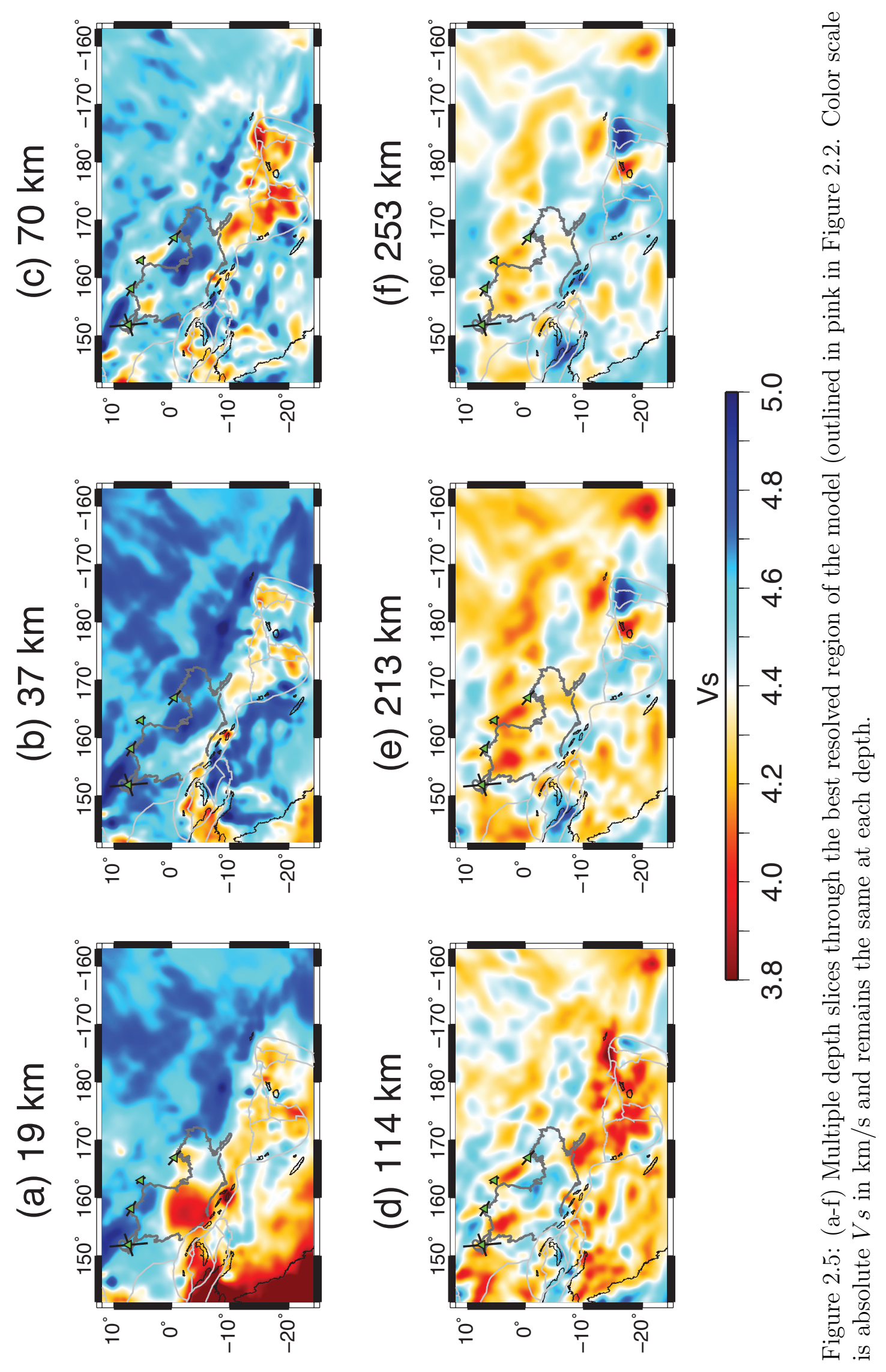


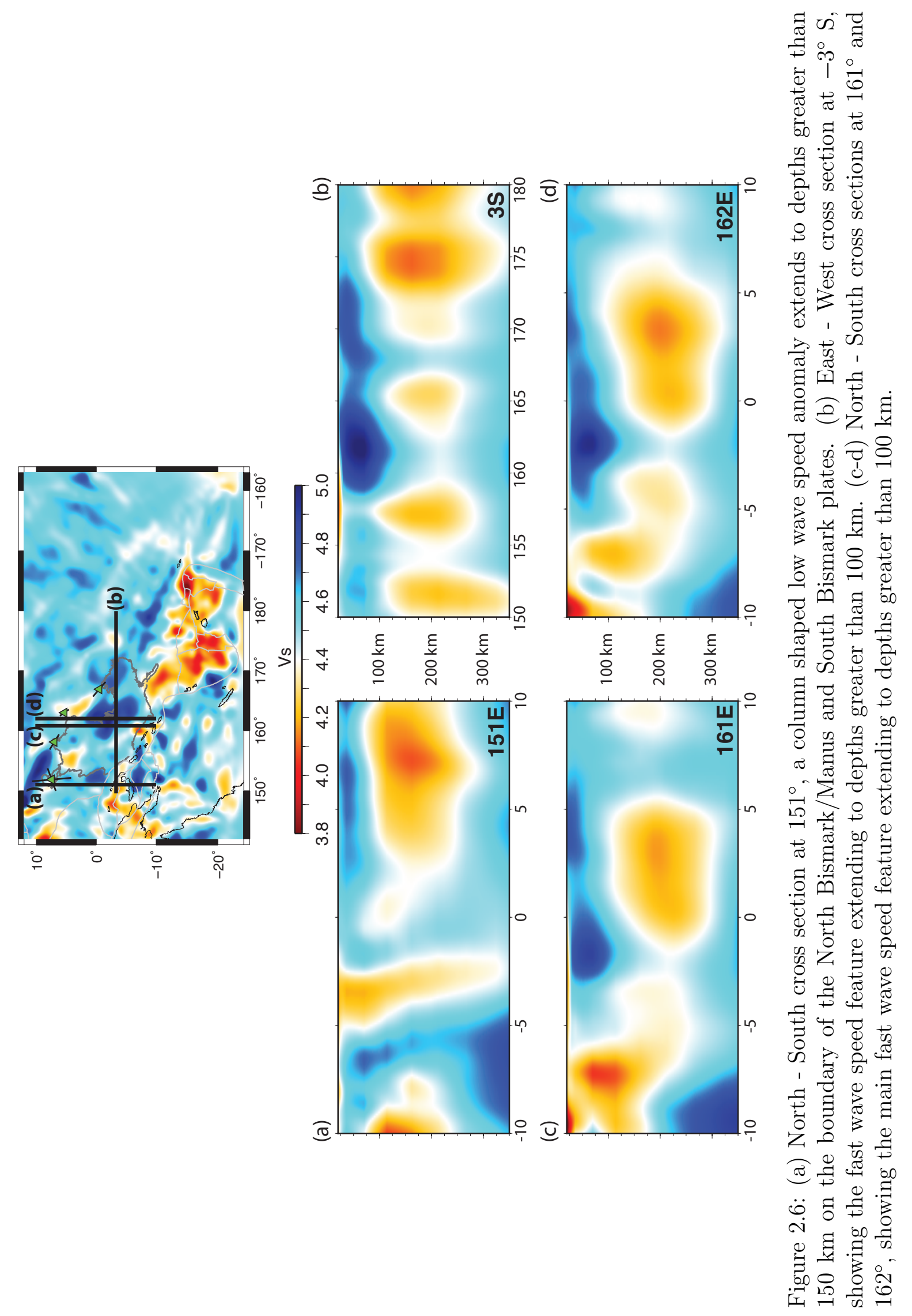




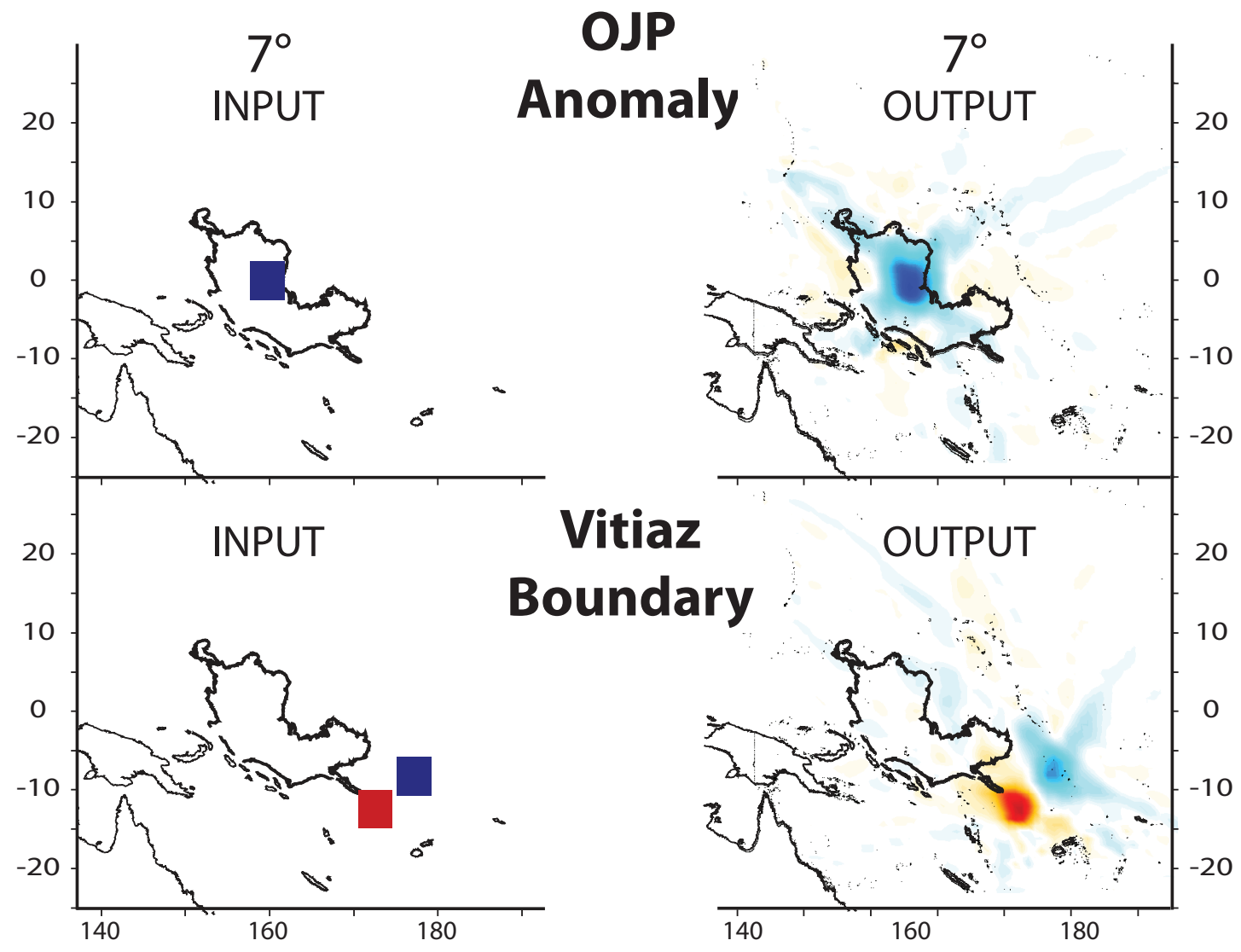

Figure 2.7: Resolution tests for a $7^{\circ}$ sized fast wave speed anomaly in the location of our observed anomaly. We are able to recover both the shape and amplitude of the fast wave speed anomaly with limited smearing. Also shown is an alternating fast and slow wave speed anomaly simulating the boundary between old, seismically fast lithosphere and young, seismically slow lithosphere. Both fast and slow anomalies are recovered with limited smearing across the boundary. 


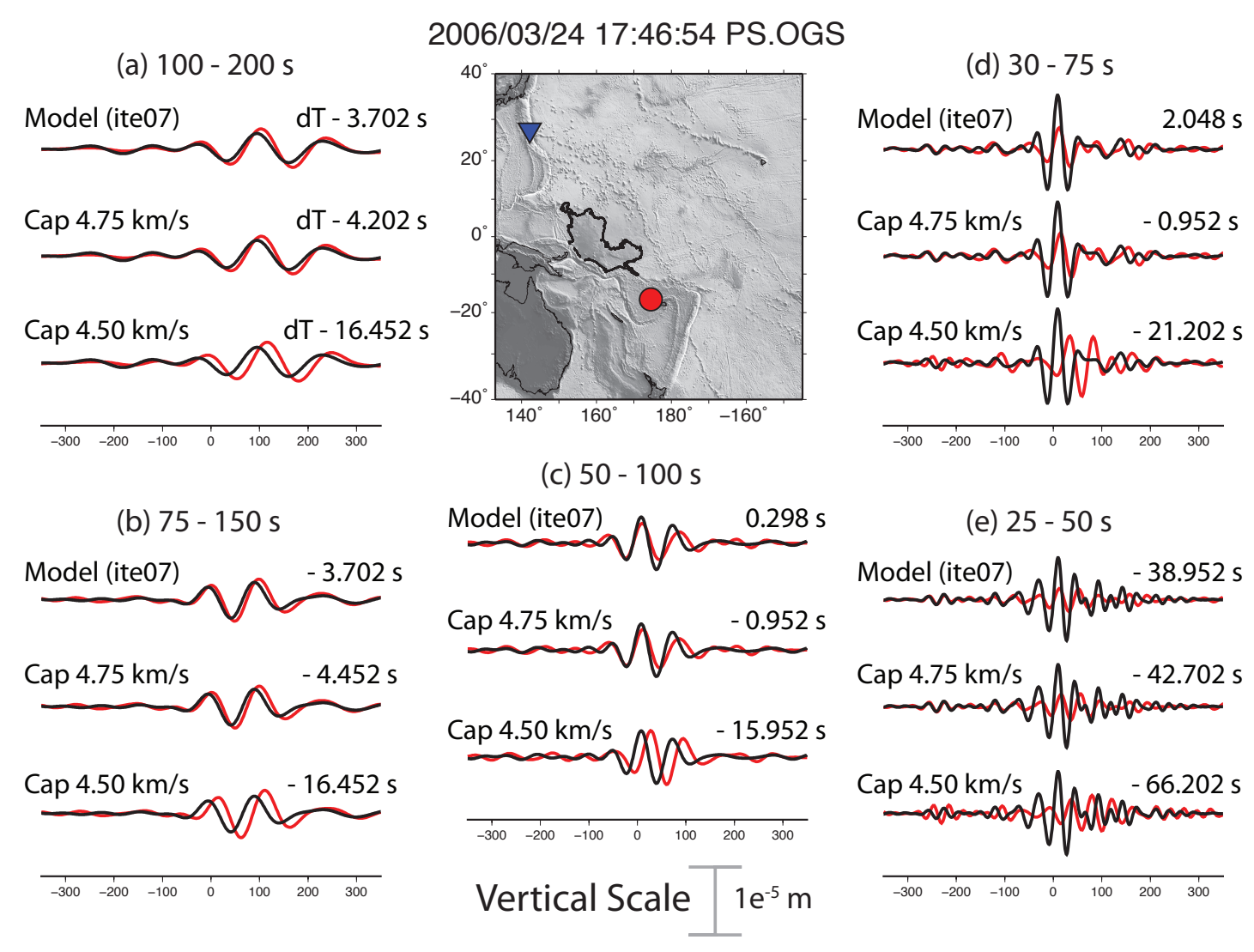

Figure 2.8: The final iteration (ite07) model is shown plotted above two capped model cases, limiting the maximum $V s$ to 4.5 and $4.75 \mathrm{~km} / \mathrm{s}$. For all frequency bands (a- e) the $4.5 \mathrm{~km} / \mathrm{s}$ model is too slow to fit the main surface wave arrival. The $4.75 \mathrm{~km} / \mathrm{s}$ model fits more closely to the observed data. Based on the capping tests, we can confidently identify a wave speed $>4.75 \mathrm{~km} / \mathrm{s}$ in the fastest portions of our model beneath the OJP. 


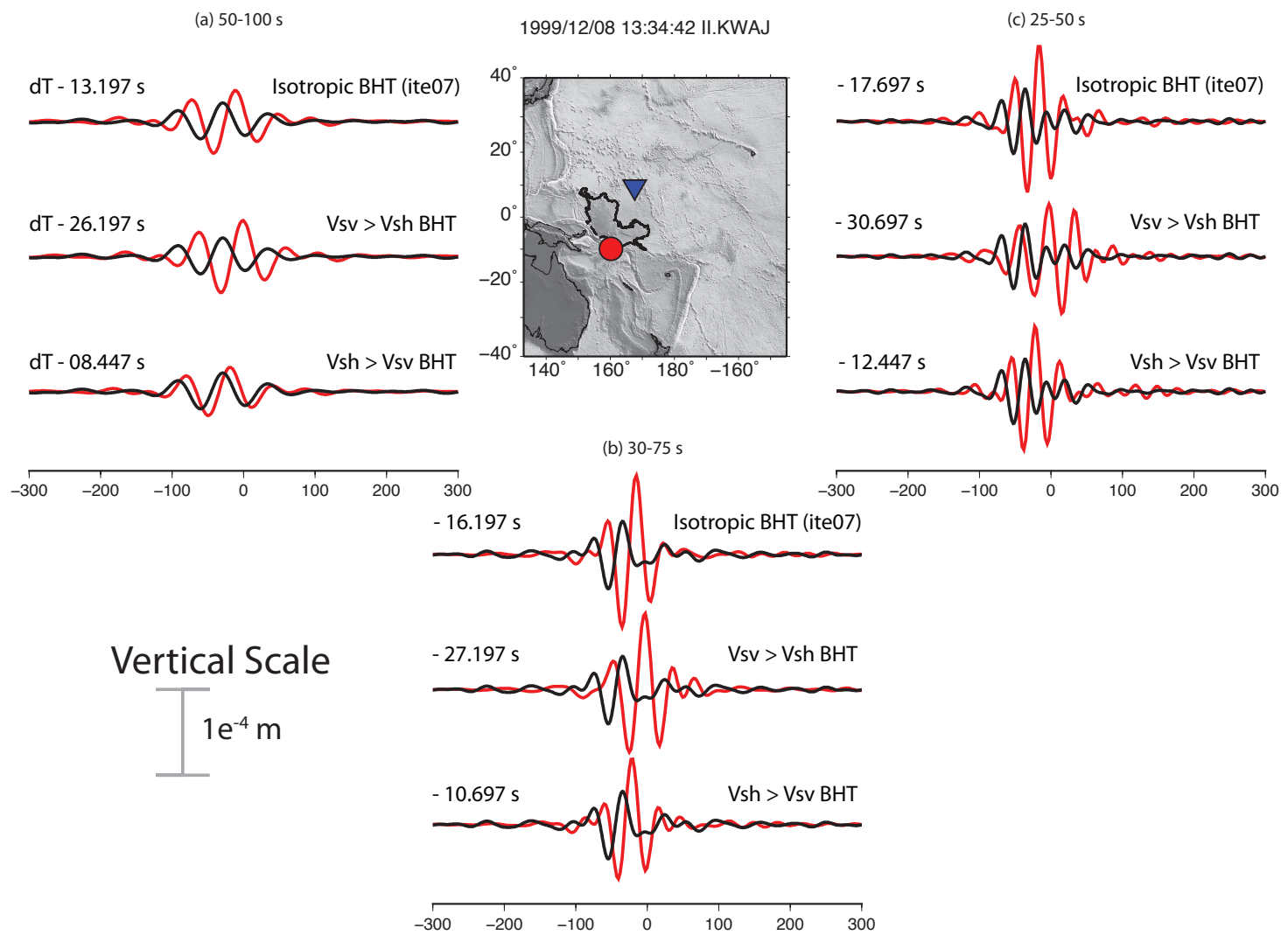

Figure 2.9: The BHT component of our final iteration (ite07) isotropic model is shown plotted above two test cases of anisotropy, a $V s_{v}>V s_{h}$ case as in Beghein et al. (2014) and a $V s_{h}>V s_{v}$ case analogous to adding PREM anisotropy to our model. The $V s_{v}>V s_{h}$ case reveals a transverse component surface wave arrival that is late compared to the data for all frequency bands (a-c). The applied PREM anisotropy case fits the data slightly better than our isotropic model, suggesting that the fast anomaly is weakly anisotropic. 


\section{Appendix}

Included in the appendix is a description of the scattering integral (SI) methodology (Zhao et al., 2005; Zhang et al., 2007; Chen et al., 2007b,a; Zhang and Shen, 2008) and additional figures and tables.

Table 2.2: $2.5 \mathrm{Gpa}$ and $1125^{\circ} \mathrm{C}$. Grossular Garnet (gr), Forsterite (fo), Enstatite (en), Diopside (di), Alpha Quartz (aqz), Almandine (alm), Pyrope (py), Ferrosilite (fs), Hedenbergite (hed), Muscovite (mu), Spinel (sp), Coesite (coe), Jadeite (jd). Jadeite (jd), the sodium bearing clinopyroxene was used in place of Omphacite. Coesite (coe) was used in place of Rutile because it was the closest mineral structure substitute. Phengite and Opaques were combined into Muscovite (mu). References HA04 refers to Hacker and Abers (2004) and Ji03 refers to Ji et al. (2003). The $m$ prefix signifies the modal abundances have been modified from the published values.

Figure 2.10: Empirical Green's Functions (EGF): a) Lines connecting a "virtual" source (red triangle) located at station IU.AFI to each receiver (black triangles). b) EGFs plotted from the "virtual" source to each receiver plotted in (a). EGFs are computed by the cross-correlation of vertical records. Waveforms have been filtered between 0.01-0.02 Hz and are plotted by distance from the "virtual" source. Figure 2.11: (a) Shows the main fast wave speed anomaly. Vs values for the constained area shown in a) are plotted as a histogram in b) to illustrate the distribution of shear wave speed at each grid point in the constrained model. The histogram shows that the majority of grid points have a $V s$ value of $<4.75 \mathrm{~km} / \mathrm{s}$. Extreme values, $>4.75$, are from the center of the anomaly. 
The scattering integral (SI) methodology constructs the station strain green tensors (SGTs) from a 3D reference model, here using a finite difference simulation, of the response to a force at a "source" location. Travel time anomalies are then measured from the observed and synthetic waveforms at each station. Station SGTs are used to calculate finite-frequency sensitivity kernels to perturbations in $V_{p}$ and $V_{s}$. The travel-time measurements and sensitivity kernels are used to invert for Earth structure which are then added to the 3D reference model and can later be repeated and iterated on. The methodology varies slightly whether using ambient noise data or earthquake data. For ambient noise data, the SGT is calculated from a Gaussian pulse located at the coordinates of another "virtual source" station. The approach using earthquake data applies the earthquake's moment tensor acting at its source location to the finite difference calculation.

From Chen et al. (2007b,a), a forward problem for displacement can be written as

$$
\delta d=A \delta m=\int K_{d}(\hat{m}, x) \cdot \delta m(x) d V(x) \approx d(m)-d(\hat{m})
$$

where $d$ is the data represented as functionals of an Earth model, $m$ and calculated for a starting Earth model, $\hat{m}$. Data sensitivity kernels, $K_{d}$, are derivatives of the data with respect to the model parameters within the $\hat{m}$ volume, $V$, at every point within the model, $x$. Generalized seismological data functionals (GSDFs) are used to map synthetic waveforms $\left(u_{i}(\omega)\right)$ into observed waveforms $\left(\bar{u}_{i}(\omega)\right)$ in the frequency domain, using two frequency-depandet quantities, the phase delay time $\left(\delta \tau_{p}(\omega)\right)$ and the amplitude reduction time $\left(\delta \tau_{q}(\omega)\right)$ and equation 2.8 below,

$$
\bar{u}_{i}(\omega)=u_{i}(\omega)^{i \omega\left[\delta \tau_{p}(\omega)+i \delta \tau_{q}(\omega)\right]}
$$

where $\delta \tau_{p, q}(\omega)$ are measured at a set of discrete frequencies, $\omega_{n}$ (Gee and Jordan, 1992; Chen et al., 2007b,a). We use only the phase delay time, $\delta \tau_{p}(\omega)$. 
Once phase anomalies are measured, perturbation kernels $\left(J_{i n}^{s r}\right)$ can be constructed for the $n t h$ misfit measurement made on the ith component of the seismogram, generated by source, $s$, and recorded at receiver, $r$, by

$$
\delta d_{i n}^{s r}=\int J_{i n}^{s r}(t) \delta u_{i}^{s}\left(x_{r}, t\right) d t
$$

where the seismogram perturbations are related to density, $\rho$, and strain, $c_{j k l m}$,

$$
\begin{aligned}
\delta u_{i}^{s}\left(x_{r}, t\right)= & -\iint\left\{\sum_{j} G_{i j}\left(x_{r}, t-\tau ; x\right) \partial_{\tau}^{2} u_{j}^{s}(x, \tau) \delta \rho(x)+\right. \\
& \left.\sum_{j k l m} \partial_{k} G_{i j}\left(x_{r}, t-\tau ; x\right) \partial_{l} u_{m}^{s}(x, \tau) \delta c_{j k l m}(x) d \tau\right\} d V(x)
\end{aligned}
$$

then using reciprocity, the sensitivity kernels of the data functionals with respect to $\rho$ and $c_{j k l m}$ are

$$
\begin{aligned}
K_{d_{i n}^{s r}}^{\rho} & =-\int d t \int d \tau J_{i n}^{s r}(t) \sum_{j} G j i\left(x, t-\tau ; x_{r}\right) \partial_{\tau}^{2} u_{j}^{s}(x, \tau) \\
K_{d_{i n}^{s r}}^{c_{j k l m}} & =-\int d t \int d \tau J_{i n}^{s r}(t) \partial_{k} G j i\left(x, t-\tau ; x_{r}\right) \partial_{l} u_{m}^{s}(x, \tau)
\end{aligned}
$$

as in Zhao et al. (2005) and Chen et al. (2007a). The forward wavefield from the source, $s$, is convolved with the Receiver Green's Tensor (RGT) from the receiver, $r$, to form the "scattering-integral" (Chen et al., 2007b,a). The misfit is then minimized via least-squares. 


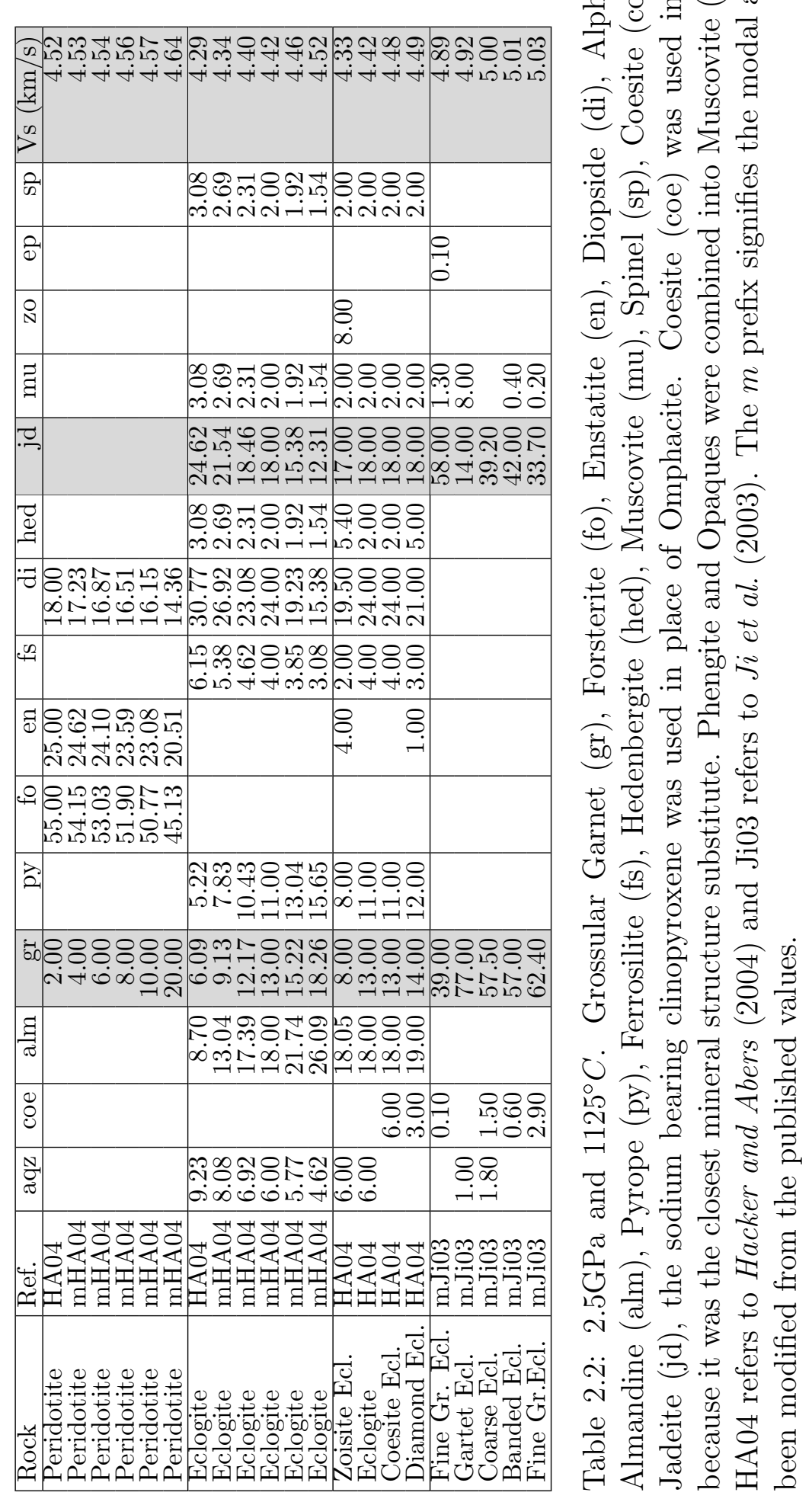


a) Virtual source, IU.AFI, to receivers

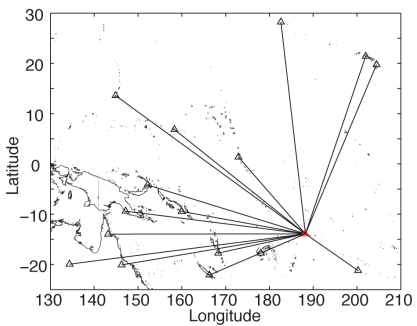

b) EGFs filtered from $0.01-0.02 \mathrm{hz}$

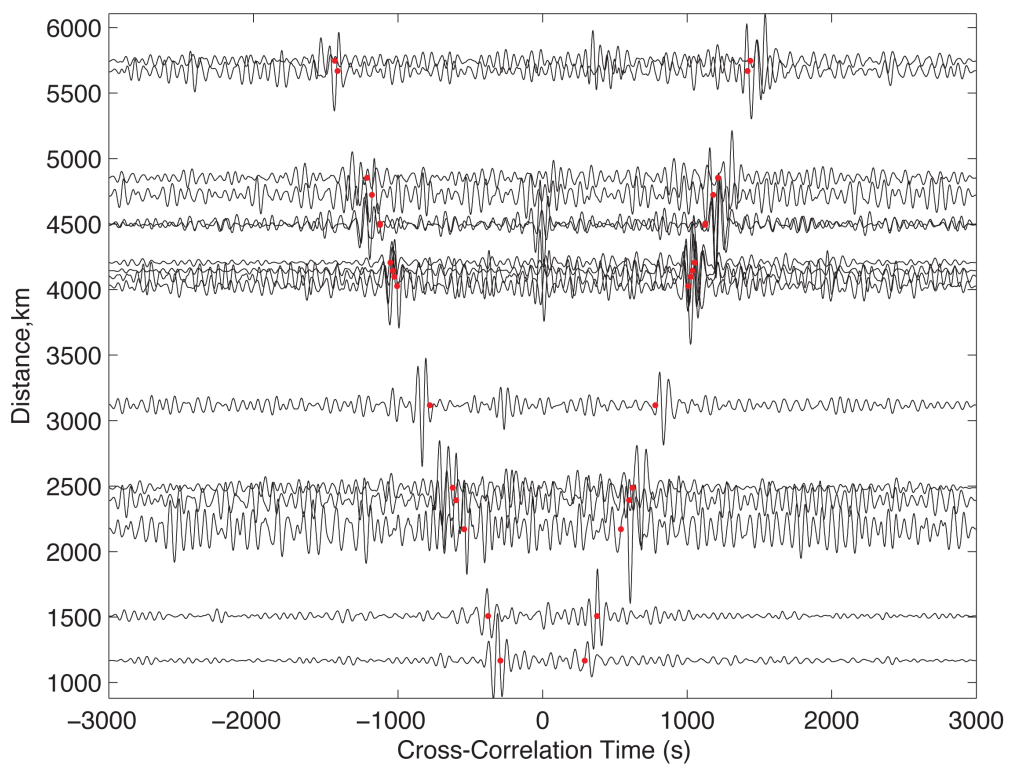

Figure 2.10: Empirical Green's Functions (EGF): a) Lines connecting a "virtual" source (red triangle) located at station IU.AFI to each receiver (black triangles). b) EGFs plotted from the "virtual" source to each receiver plotted in (a). EGFs are computed by the cross-correlation of vertical records. Waveforms have been filtered between $0.01-0.02 \mathrm{~Hz}$ and are plotted by distance from the "virtual" source. 


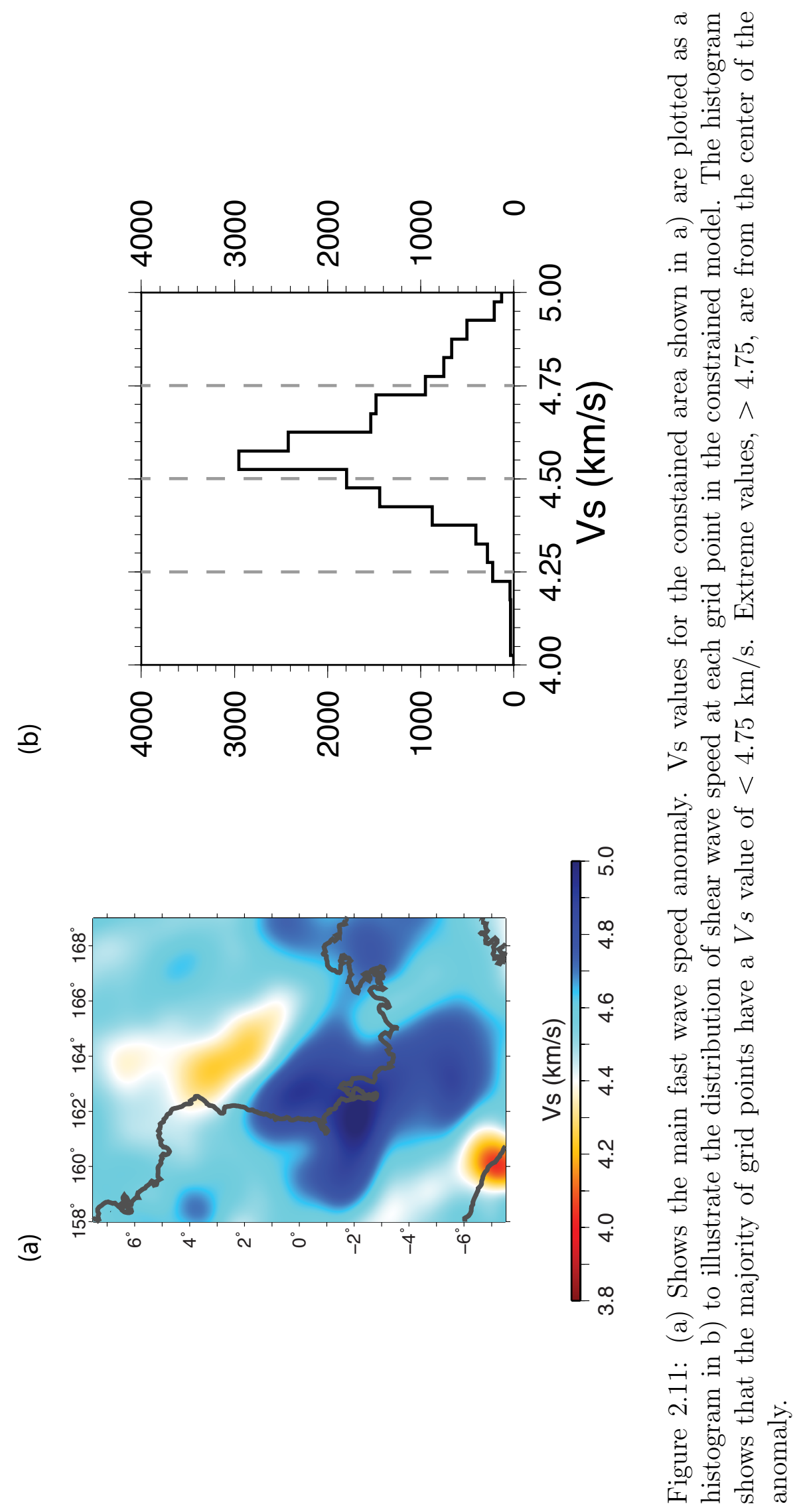




\section{CHAPTER 3}

\section{Wave speed structure of the eastern North American margin}

by

Brian M. Covellone ${ }^{1}$; Brian Savage ${ }^{1,2}$; Yang Shen ${ }^{1}$

${ }^{1}$ Graduate School of Oceanography, University of Rhode Island, Narragansett, RI 02882

${ }^{2}$ Department of Geosciences, University of Rhode Island, Kingston, RI 02881 


\begin{abstract}
The eastern North American margin (ENAM) is the result of nearly a billion years of continental collision and rifting. Thick continental lithosphere of the North American craton makes up the western boundary of our study region. Transitions in lithosphere thickness occur at the intersection of the craton and the Atlantic ocean basin. A dramatic change in lithosphere thickness at this boundary may drive asthenosphere upwelling along the edge of the continent. We observe a continuous low wave speed feature at the edge of the continent for depths between 126 and $187 \mathrm{~km}$. Additionally, a large slow wave speed anomaly beneath New England continues offshore aligned with the New England Seamount chain, and is possibly a remnant of the Monteregian hot spot active 100 - 120 Ma.
\end{abstract}

\title{
Introduction
}

The eastern North American margin (ENAM) is presently a passive continental margin and the result of multiple episodes of continental collision and rifting dating back greater than a billion years ago (1 Ga) (Hoffman, 1988, 1991; Thomas, 2006). Thomas (2006) describes two complete Wilson cycles, a cycle of closing and opening of ocean basins associated with the assembly and breakup of supercontinents, that have shaped the ENAM. The first Wilson cycle that assembled Rodinia (the Grenville Orogen) and its subsequent breakup created the Iapetus Ocean between $1.35-0.53 \mathrm{Ga}$ (Thomas, 2006). A second Wilson cycle began with the closing of the Iapetus Ocean and assembly of Pangea from the successive Taconic, Acadian and Alleghanian Orogenies (commonly referred to as the Appalachian-Ouachita Orogen) beween 495 - 270 million years ago (Ma) (Thomas, 2006). Early Jurassic northeast striking rift structures place the break-up of Pangaea approximately 230 Ma in present day southeastern North America (Schlische, 2003).

The initial rifting and breakup of Pangea and formation of the Atlantic basin 
are associated with multiple magmatic provinces scattered on both margins of the present day Atlantic basin. Two in particular, the Central Atlantic Magmatic Province (CAMP) and the East Coast Margin igneous province (ECMIP) have been suggested as a result of either 1) a mantle plume or 2) continental rifting in the form of $2 \mathrm{a}$ ) reactivation of Paleozoic structures or $2 \mathrm{~b}$ ) upwelling convection cells at the edges of cratons (White et al., 1987; White and McKenzie, 1989; Holbrook and Kelemen, 1993; Oyarzun et al., 1997; Wilson, 1997; McHone, 2000; Janney and Castillo, 2001; Puffer, 2003; Nomade et al., 2007; Beutel, 2009).

A complex history of collision and breakup recorded on the continent and thick post rifting sediments along the Atlantic margin make unraveling the broad scale features in the lithosphere difficult through either direct sampling of material or active source seismology. Moreover, the resolution of wave speed models for North America vary widely across the continent and are primarily long wavelength, greater than $5^{\circ}$. Regardless, interpretations of the current state and origins of the ENAM have been made from various continental (Van der Lee and Nolet, 1997; Goes and van der Lee, 2002; Godey et al., 2003; Van der Lee and Frederiksen, 2005) and regional (Li et al., 2002, 2003; Rychert et al., 2005, 2007; Liang and Langston, 2009; Parker et al., 2013) scale seismic studies. The current station distribution of seismic networks deployed throughout the United States, as well as stations located in northeastern Canada and throughout the Caribbean, provide ideal coverage for ambient noise tomography of the ENAM to vastly improve the resolution of wave speed structures of the ENAM in a single wave speed model.

The wave speed model presented here provides new and valuable insight into the crust and upper mantle $(<350 \mathrm{~km})$ seismic wave speed structure of the ENAM including the extent and depth of a deformation throughout the lithosphere from rifting and plate tectonic forces. By taking advantage of the early stages of EarthScope's Transportable Array (TA) of seismic stations, our model provides much 
higher resolved features than previous continent scale models.

\section{Methodology}

To determine the 3-dimensional seismic wave speed structure beneath the Eastern North American Margin (ENAM) we use a iterative, finite-frequency tomography approach using full-waveform ambient noise data. Green's functions are derived from continuously recorded broadband seismic data at periods up to 200 seconds. Use of Green's functions from ambient noise exploits 20 plus years of broadband seismic data recorded at stations throughout North and Central America and the Caribbean, not reliant on earthquake distributions and solving for the earthquake source mechanism. The use of data from ambient noise reduces a source of error, from an earthquake's location and source mechanism, and is able to exploit smaller temporary seismic station deployments unable to gather sufficient earthquake data during their deployment window. We measure surface waves sensitive to crust and upper mantle $(<500 \mathrm{~km})$ Earth structure, key to understanding the nature of the margin. A detailed description of the methodology can be found in Chapter 2 of this volume. Here we will summarize the most important steps.

Continuous, vertical component seismic data recorded between 1990 and 2014, was gathered from IRIS DMC for 203 stations located in the eastern United States, Caribbean, Central and South America (Figure 3.1). To extract usable Rayleigh wave signals from the raw data, an ambient noise processing procedure outlined in Shen et al. (2012) and Gao and Shen (2014) was used. After removing the instrument response a frequency time normalization (FTN) (Shen et al., 2012) was used to normalize the data. Earthquake signals are removed and a cross correlation between station pairs is calculated with one station acting as the "virtual" source. The cross correlated records are stacked and following a derivative, represent our empirical Green's functions (EGFs). 
Synthetic seismograms are calculated by propagating seismic waves from a virtual source to each receiver using a nonstaggered-grid finite-difference method (Zhang et al., 2012). As in Chapter 2, the initial model is a combination of the global surface wave diffraction model, CUB (Ritzwoller et al., 2002), and AK135 (Kennett et al., 1995) at depths greater than $396 \mathrm{~km}$.

EGFs and synthetics are filtered using a two-pass butterworth filter at five overlapping finite frequency bands, $200-100 s, 150-75 s, 100-50 s, 75-30 s$, $50-25 s$. Phase delays, $d T$, were measured between the data and synthetics by cross-correlation for each frequency band. Low quality signals are removed from the measurement using a minimum signal-to-noise ratio and a minimum cross correlation coefficient criteria (Table 3.1). Iteration 1 (ite01) measured 17,624 station pairs, resulting in 53,186 total measurements. The scattering-integral (SI) approach (Zhao et al., 2005; Zhang et al., 2007; Chen et al., 2007b,a; Zhang and Shen, 2008) is used to construct strain green tensors (SGTs) and calculate finitefrequency sensitivity kernels. The phase delay, $d T$, measurements and sensitivity kernels are used to invert for perturbations in $V p$ and $V s$ which are then added to the $3 \mathrm{D}$ reference model at the end of each iteration (see Chapter 2 Appendix for details).

\section{Results}

\section{Resolution and data fit}

Along the ENAM an extensive network of seismic stations is ideal for use in ambient noise tomography, Figure 3.1. For the given station distribution, within the continental interior our resolving ability is excellent. However, stations off of the continent are limited to Bermuda (IU.BBSR) and the Caribbean, resulting in a reduction of resolving power of offshore features. The inclusion of stations

in Bermuda and throughout the Caribbean increases coverage offshore into the Atlantic ocean but a lack of crossing paths hinders our ability to resolve fine scale 
features. To demonstrate the resolving capabilities of our dataset, Figure 3.2 shows the computed model domain perturbed with a $1^{\circ}, 3^{\circ}, 5^{\circ}$, and $7^{\circ}$ sized harmonic pattern of positive and negative $5 \%$ wave speed anomalies.

Resolution is excellent on the North American continent and we are able to recover anomalies of $3^{\circ}, 5^{\circ}$, and $7^{\circ}$ in size with little to no smearing or lose in amplitude to depths $>300 \mathrm{~km}$. We are also able to resolve features at $1^{\circ}$, but at a significant loss of amplitude. Off of the continent, anomalies $5^{\circ}$ or larger can be interpreted approximately $500 \mathrm{~km}$ offshore into the Atlantic ocean and $7^{\circ}$ resolution is obtained throughout the Caribbean.

These resolution tests show that wave speed structure is best resolved between depths of approximately 30 and $300 \mathrm{~km}$, similar to as seen in Chapter 2 of this volume and in Gao and Shen (2014) using a similar methodology and period range.

Histograms showing the range of $d T$ and number of measurements for each iteration is shown in Figure 3.3. After a single iteration, an overall reduction in traveltime and increase in the number of measurements indicates the model is improving and is representing large and small scale Earth structures.

\section{Shear wave speed structure}

Seismic wave speed structure is plotted in Figure 3.4 as absolute wave speed in $\mathrm{km} / \mathrm{s}$ and as perturbation relative to the initial model, CUB (Ritzwoller et al., 2002), in Figure 3.5. Our initial iterations show the data requires a larger contrast between the fast cratonic lithosphere of the Grenville Province and the slower Appalachians in the northwestern portion of our model. This contrast is strongest within the crust and uppermost mantle. Broadly, the North American east coast has lower wave speeds than inland areas at depths $>100 \mathrm{~km}$ primarily caused by the difference in ocean/continent lithosphere thickness. At depths $<100 \mathrm{~km}$, the mantle is rather homogeneous across the ocean/continent boundary with the exception of a number of small scale features discussed later. The shallowest depth, 
$37 \mathrm{~km}$ in Figure 3.4 is dominated by the crustal thickness differences between the ocean and continent. One notable exception is the Appalachians, which are characterized by slower wave speeds from Pennsylvania southward to Tennessee at depths up to $50 \mathrm{~km}$.

Within the upper mantle, New England (New Hampshire, Vermont, Massachusetts, Rhode Island and Connecticut) has a prominent low wave speed anomaly from the Hudson River, northeast through Vermont and New Hampshire and east through Massachusetts that continues offshore towards the New England Seamounts. This low wave speed feature is present between about $50-250 \mathrm{~km}$ into the mantle, and is similar in shape and extent to the observed anomalies in Van der Lee and Nolet (1997), Levin et al. (2000), Menke and Levin (2002), Li et al. (2003), Van der Lee and Frederiksen (2005). A fast wave speed region, at $50-70 \mathrm{~km}$ beneath Lake Ontario and the Adirondack Mountains shown in Li et al. (2003), is located more to the north and east in our model.

Along $34^{\circ}-36^{\circ} \mathrm{N}$ a number of lower wave speed, isolated anomalies are present. A small-scale, but prominent anomaly appears beneath southern Maryland and Virginia at depths greater than $50 \mathrm{~km}$, this feature is also observed in Van der Lee and Nolet (1997) and Van der Lee and Frederiksen (2005). This anomaly gets stronger and grows in scale at deeper depths. To the west, the mantle beneath the Appalachian Mountains have low wave speeds from Pennsylvania through Tennessee and throughout the crust. In the upper mantle (> $50 \mathrm{~km})$ a continuous low wave speed anomaly in West Virginia extends to depths greater than $250 \mathrm{~km}$. A low wave speed region is present just north of the New Madrid Seismic Zone, only between $60-90 \mathrm{~km}$.

In southern North America a low wave speed anomaly along the coast of South Carolina and Georgia extends from $30-200 \mathrm{~km}$ into the mantle; this feature is only observed in Van der Lee and Nolet (1997) and Van der Lee and Frederiksen (2005) 
model at depths greater than $100 \mathrm{~km}$. Similar to Van der Lee and Nolet (1997) and Van der Lee and Frederiksen (2005), a low wave speed region is also observed up Florida's gulf coast, with the highest amplitudes located to the southeast of the Florida panhandle.

Between approximately $120-190 \mathrm{~km}$ depth the southern coastal anomalies converge into a single anomaly aligned along the North American continent margin as nearly continuous low wave speed structure from Florida to Maryland. This is in contrast to global shear wave tomography models TX2000 and TX2011 (Grand, 2002) that show a much simpler structure between the continental and oceanic lithosphere, absent the low wave speeds observed in our model.

\section{Discussion}

Our resulting wave speed model shows features broadly similar to other regional (Li et al., 2002, 2003; Rychert et al., 2005, 2007; Liang and Langston, 2009; Parker et al., 2013) and continent (Van der Lee and Nolet, 1997; Goes and van der Lee, 2002; Godey et al., 2003; Van der Lee and Frederiksen, 2005) scale wave speed models encompassing the ENAM. The seismically fast regions in Canada and to the northwestern portion of our model reflect North American cratonic lithosphere and the $1.3-0.8 \mathrm{Ga}$ Grenville Province (Hoffman, 1988; Rivers et al., 1989). At depths in the model less than $50 \mathrm{~km}$ the wave speed structure is generally a reflection of crustal thickness across the ocean continent margin. Within the upper mantle, Figure 3.4 (e) at $126 \mathrm{~km}$, thinner lithosphere and seismically slower wave speeds are observed along the Atlantic coast and thicker crust with faster wave speeds are observed in the continental interior including the Appalachians and Grenville Province ( Li et al., 2002). A region of low wave speed beneath the southern Appalachians is consistent with thickened crust, greater than $50 \mathrm{~km}$, in the region observed by Hawman (2008) and Parker et al. (2013). Ekström (2014) 
observed very slow phase velocities at short periods, $5-20$ seconds, along the Gulf Coast likely the result of a thick sedimentary layer. Our resolution is limited at such shallow depths and throughout the Gulf Coast, but we do not observe this broad slow feature in our model. As observed in Van der Lee and Nolet (1997) and Van der Lee and Frederiksen (2005), south and east of the craton is dominated by a series of isolated low wave speed anomalies that will be discussed below.

The most discernible low wave speed anomaly is beneath New England and continuing offshore in the direction of the New England Seamounts. Li et al. (2002) described this anomaly as a result of past heating of the lithosphere from the Monteregian hotspot passing through the region 100-120 Ma; consistent with interpretations by Van der Lee and Nolet (1997). Li et al. (2002) also points out that the New England anomaly is unlikely to be a result of water in the mantle, as suggested by Van der Lee and Nolet (1997), as water would have likely partitioned into the melt during the Jurassic opening of the Atlantic Ocean. Goes and van der Lee (2002) calculated the thermal structure of North America by inverting $V p$ and $V s$ values from continental scale $V s$ (Van der Lee and Nolet, 1997) and Vp (Bijwaard and Spakman, 2000) tomography models. Goes and van der Lee (2002) calculated a mantle potential temperature beneath New England of around $1200^{\circ} \mathrm{C}$ at $100 \mathrm{~km}$ depth, significantly warmer than the average Eastern North America geotherm. The calculated geotherm and lower wave speeds are consistent with a thinner continental lithosphere as imaged by Rychert et al. (2005).

In our wave speed model the New England anomaly is present between about $50-250 \mathrm{~km}$. The location and extent of the anomaly is consistent with Van der Lee and Nolet (1997), Levin et al. (2000), Menke and Levin (2002), Li et al. (2003), and Van der Lee and Frederiksen (2005) and its association with the New England Seamount chain offshore is tough to discount. In cross section, Figure 3.6, the low wave speed anomaly shallows seaward. The slowest wave speeds are 
seen adjacent to the craton to the west and at approximately $200 \mathrm{~km}$ depth. As suggested in Li et al. (2002), this low wave speed anomaly may be a result of thermal erosion associated with the Monteregian hotspot or asthenospheric melt or volatiles (Rychert et al., 2005). To test whether a thermal anomaly could persist for $100 \mathrm{Ma}$, we calculate a diffusion time, $t$, using a scaled version of the diffusion equation, where $t \approx \frac{\text { radius }^{2}}{\kappa}$. Two values for the diffusivity (Gibert et al., 2003), $\kappa$, were used and a suite of sizes for the radius, see Table 3.2. The time needed to diffuse a thermal anomaly is longer than the $100-120$ Ma since the Monteregian hotspot for all cases except the $50 \mathrm{~km}$ radius anomaly, which significantly underestimates the size of our observed low wave speed feature.

The observed slow wave speeds beneath South Carolina, Georgia and continuing offshore roughly align with the locations of the Brunswick and East Coast magnetic anomalies (Austin et al., 1990; Holbrook et al., 1994). We observe a low wave speed feature spanning $-81^{\circ}$ to $-76^{\circ}$ longitude, dipping seaward and to depths extending from the crust to greater than $200 \mathrm{~km}$. We do not have the shallow resolution, $<35 \mathrm{~km}$, to compare our observations directly with Austin et al. (1990) and Holbrook et al. (1994) but the seaward dipping nature of the feature may imply the shallow structure is related to the deeper low wave speed anomalies we observe in our model. Austin et al. (1990) and Holbrook et al. (1994) described this region offshore as being characterized by seaward-dipping reflections, high seismic velocities $(V p 6.5-7.5 \mathrm{~km} / \mathrm{s})$ and high densities $\left(2870-3090 \frac{\mathrm{kg}}{\mathrm{m}^{3}}\right)$ indicating a margin that is highly volcanic. They describe a transitional crust, in between the rifted continent crust and new oceanic crust, that is $24 \mathrm{~km}$ thick and accreted to the margin during rifting. Any connection between our observed low wave speed anomalies and the volcanic transitional crust would have to be persistent in the mantle since rift initiation 230 Ma.

Another possible explanation for the observed low wave speed anomalies in the 
south is that they are related to small scale, edge-driven convection on the edges of continents (Till et al., 2010), reflecting either a temperature anomaly or melt. Abrupt lateral changes in lithosphere thickness, as observed on the ENAM, in combination with normal plate motion causing a "mantle wind", has been shown to drive convection and asthenospheric upwelling (Till et al., 2010). "Hot" cells have also been hypothesized to be a result of the insulating effects of continents and the absence of subduction related cooling causing localized upwelling (Gurnis, 1988; Anderson et al., 1992; Holbrook and Kelemen, 1993). Seen in Figure 3.4 at depths between $126-187 \mathrm{~km}$ and in cross section (Figure 3.7), is a continuous low wave speed feature that follows the edge of the North American continent. The low wave speeds are located in a "transitional" zone, or gap, between the thicker continental lithosphere and $\sim 230$ Ma oceanic lithosphere. Further north, this gap between the oceanic and continental lithospheres decreases in size and the transition becomes smoother. Higher amplitude anomalies of low wave speeds adjacent to South Carolina, Georgia, Delaware and Florida may be exploiting weakness in the lithosphere from past episodes of volcanism.

Lizarralde et al. (2007) observed short-length scale variability in the style of rifting in the Gulf of California and attributed these changes to inherited mantle fertility and hydration; where wider, magma-poor rifts were from depleted mantle and narrower, magma-rich rifts from fertile mantle. Moreover, the crustal structure observed across the Carolina trough (Holbrook et al., 1994) and the Guaymas segment in the Gulf of California (Lizarralde et al., 2007) were both shown to have thickened, high velocity crust from magma-rich rifting. Following Lizarralde et al. (2007), this may suggest the Carolina segment was more fertile and/or hotter than surrounding areas during rifting. This interpretation is consistent with the low wave speed anomalies reflecting weaker, rifted lithosphere that is more susceptible to plate tectonics forces and/or temperature variations. 
Further, Lizarralde et al. (2004) described abrupt changes in the velocity gradient in the mantle and crustal thickness changes as a result of changes in the spreading rate. The difference in transition style between the continental and oceanic lithosphere from south to north up the coast of North America, may be reflecting changes in the spreading rate or style of spreading as the margin began rifting. While this is appropriate for shallow structure, the deeper observed anomalies in our model are likely due to a different, present day deformation in the mantle.

\section{Conclusions}

We image the seismic wave speed structure of the ENAM using data from EarthScope's transportable array (TA) deployment in addition to other permanent and temporary networks throughout the United States, Canada, Central America and the Caribbean. Our model has excellent resolution of less than $3^{\circ}$ for the ENAM region and resolution greater than $7^{\circ}$ in the Atlantic ocean and Caribbean.

Our imaged wave speed structure is consistent with previous continent and regional scale tomography models for depths of $30-300 \mathrm{~km}$. We observe a distinct transition from the fast wave speed Grenville Province to a low wave speed feature beneath New England that is likely related to the Monteregian hot spot. Nearly continuous low wave speeds on the edge of the continent, between 126-187 km, are consistent with numerical modeling by Till et al. (2010), which describes edge-driven asthenosphere upwelling due to abrupt lateral changes in lithosphere thickness. Cells of higher amplitude anomalies, reflecting lower wave speed, are observed off of the coast of South Carolina and Delaware that may represent centers of upwelling convection cells. The transition from continental to oceanic lithosphere varies with latitude, this may be a result of changes in the spreading rate from the southern to the northeastern portion of the margin. 
Our wave speed model represents a significant improvement in resolution from previous work and provides a good starting model for future studies that plan to fully exploit the entire deployment window the EarthScope's TA. 


\begin{tabular}{|c|c|c|c|c|c|c|}
\hline Iteration & Data Type & Min CC & Min SNR & $\begin{array}{c}\text { dT Threshold } \\
\text { (seconds) }\end{array}$ & Damping & Smoothing \\
\hline 1 & AN & 0.55 & 4 & 35 & 16 & 16 \\
2 & AN & 0.55 & 4 & 35 & 8 & 12 \\
\hline
\end{tabular}

Table 3.1: Summary of measurement and inversion parameters. Minimum cross correlation coefficient (Min CC), minimum signal-to-noise ratio (Min SNR) and maximum phase delay (dT) were used to screen out low quality signals.

\begin{tabular}{|c|c|c|}
\hline Radius $(\mathrm{km})$ & Diffusion Time, $\kappa 0.01(\mathrm{Ma})$ & Diffusion Time, $\kappa 0.018(\mathrm{Ma})$ \\
\hline 50 & 79 & 44 \\
100 & 317 & 176 \\
150 & 713 & 396 \\
200 & 1268 & 1586 \\
300 & 2854 & 1586 \\
400 & 5074 & 2819 \\
\hline
\end{tabular}

Table 3.2: A scaled version of the diffusion equation, where $t \approx \frac{\text { radius }^{2}}{\kappa}$, was used to estimate the time needed to completely remove a thermal anomaly given a suite of anomaly radius sizes. Two diffusivity, $\kappa$, values were used, $0.01 \frac{\mathrm{cm}^{2}}{\mathrm{~s}}$ and 0.018 $\frac{\mathrm{cm}^{2}}{\mathrm{~s}}$ (Gibert et al., 2003). Diffusion time is given in million years (Ma). 


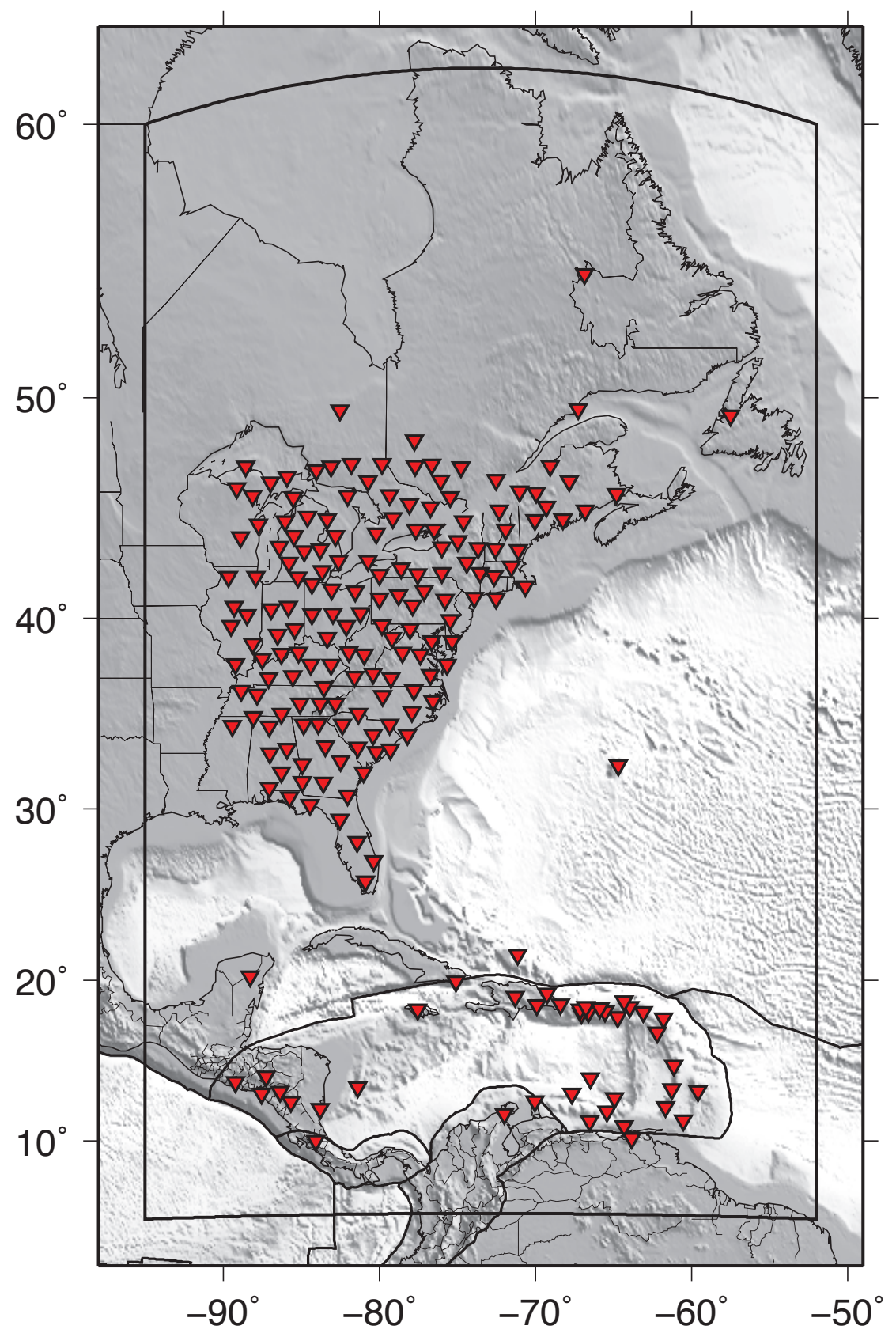

Figure 3.1: 203 Seismic stations (inverted triangles) used for this study of the Eastern North American Margin (ENAM). Our computational domain is outlined in black. Modern plate boundaries are outlined in black. 


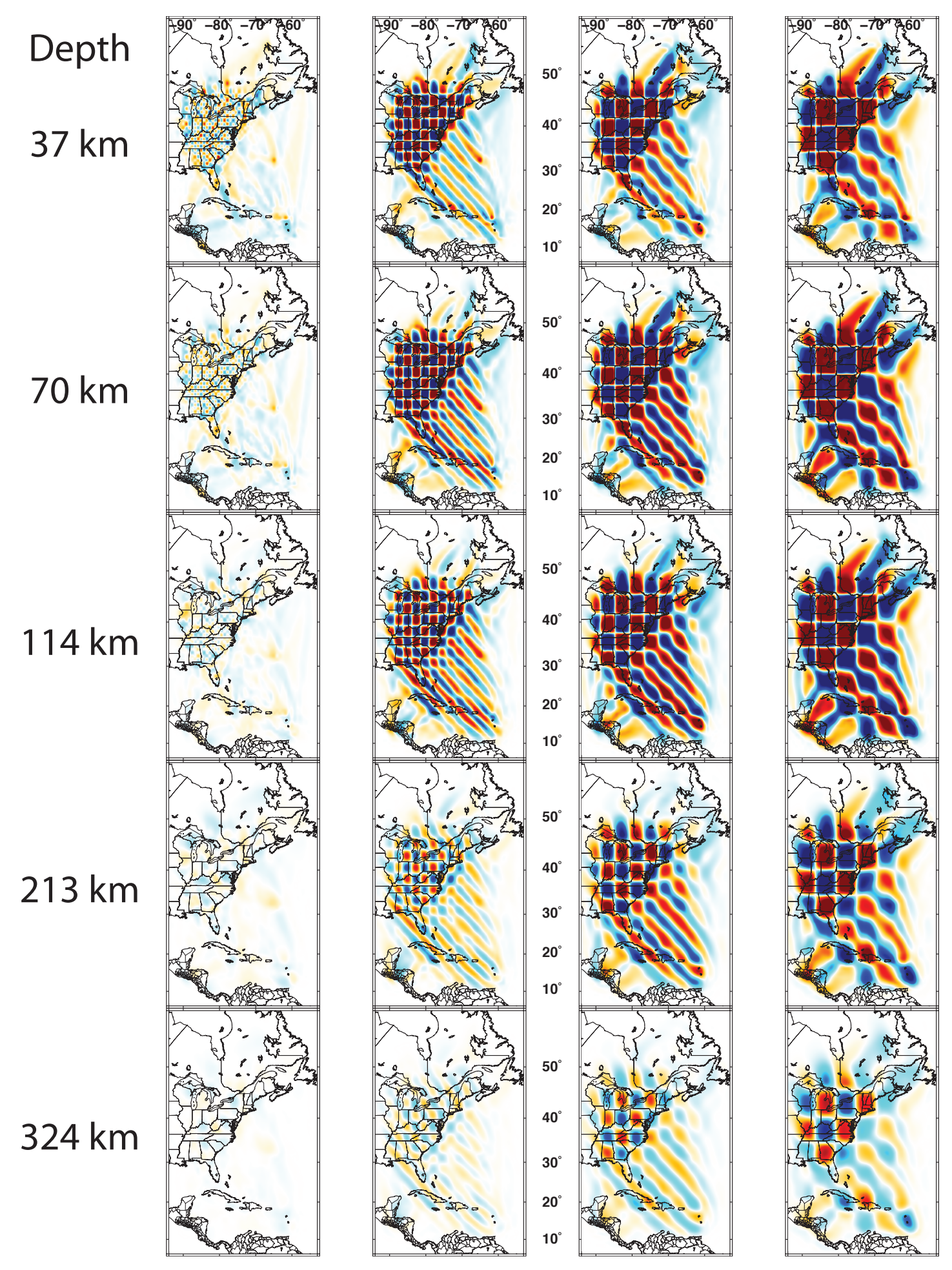

Figure 3.2: $1^{\circ}, 3^{\circ}, 5^{\circ}$ and $7^{\circ}$ sized harmonic pattern of positive and negative $5 \%$ wave speed anomalies. We are able to recover the shape of the $1^{\circ}$ sized anomalies for depths shallower than $100 \mathrm{~km}$ however there is a large decrease in amplitude. Anomalies $3^{\circ}$ and larger are very well resolved in both shape and amplitude for the all of the eastern North American margin and approximately $500 \mathrm{~km}$ offshore. Anomalies of $7^{\circ}$ or larger can be interpreted for much of the Atlantic ocean and Caribbean. 
(a)

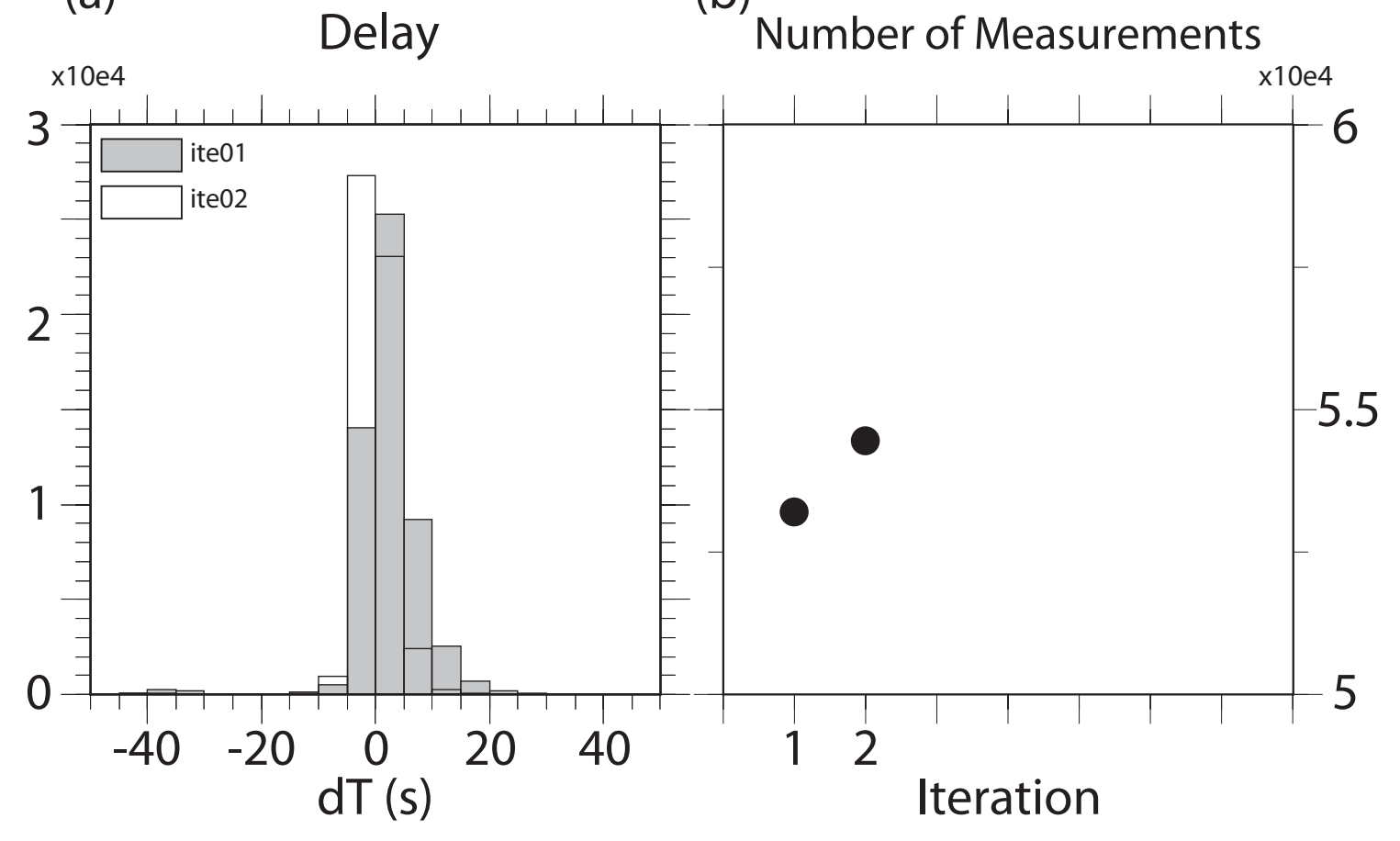

Figure 3.3: (a) Histogram of the phase delay, $d T$, measured between the data and synthetic waveforms. In grey is iteration 01, outlined in black is iteration 02. (b) Shows the number of measurements plotted with iteration. An increase in the number of measurements indicates that the model is better able to fit the data and more measurements fall within the minimum acceptance criteria. 

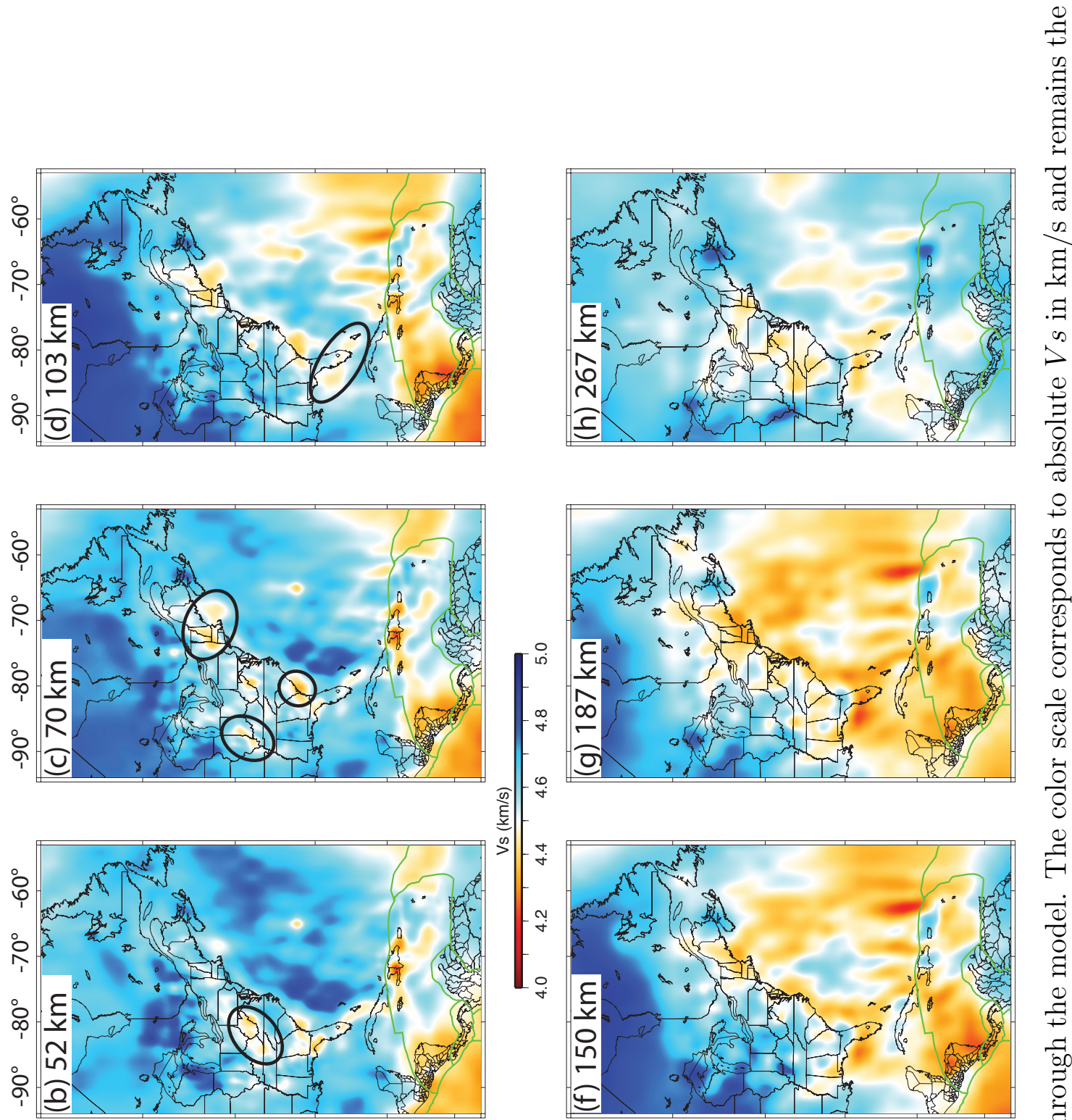

0

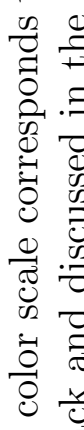
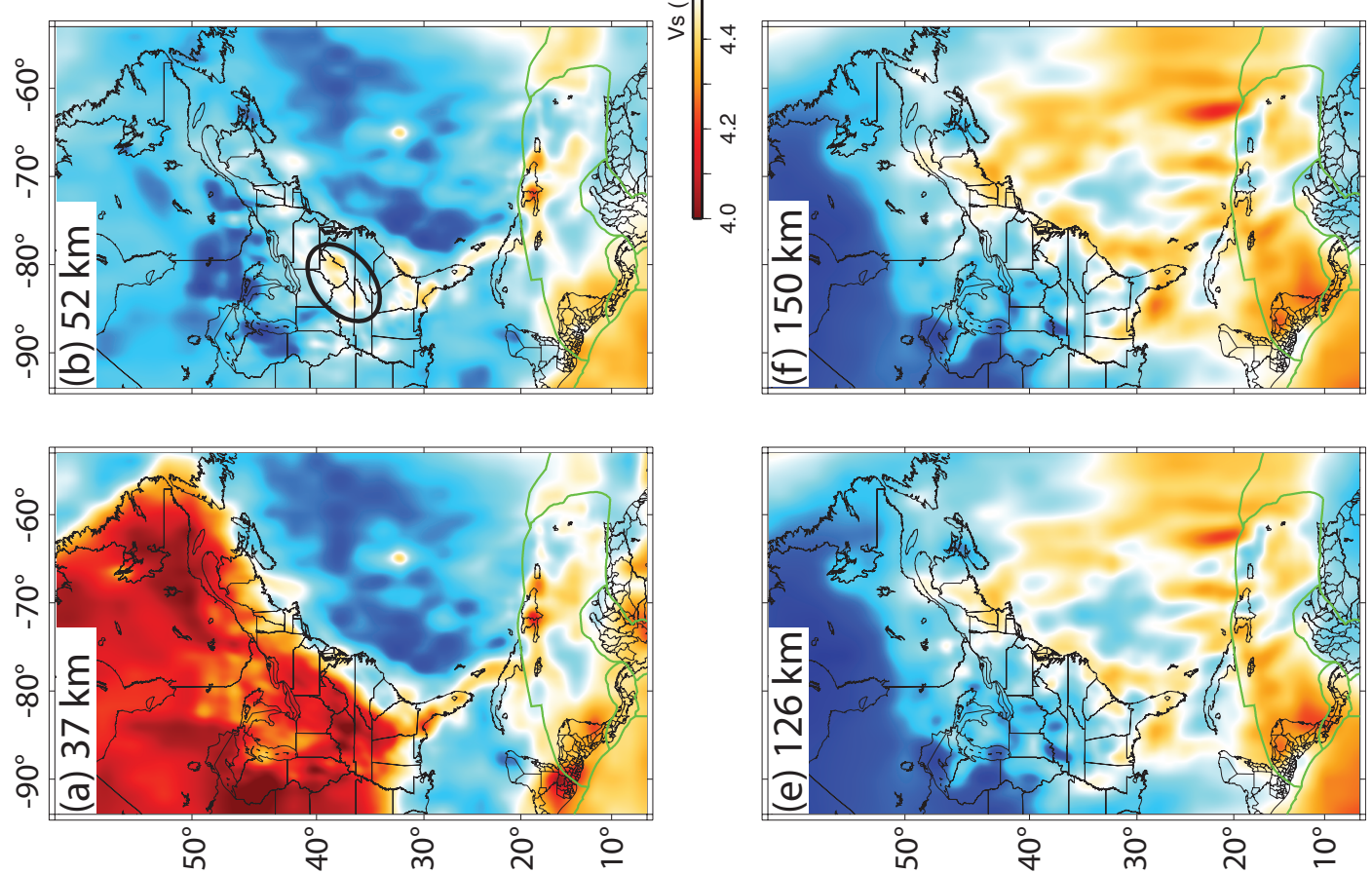

응

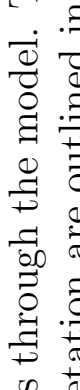

过

공

웡

党

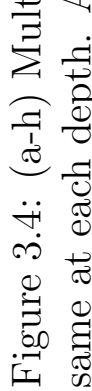



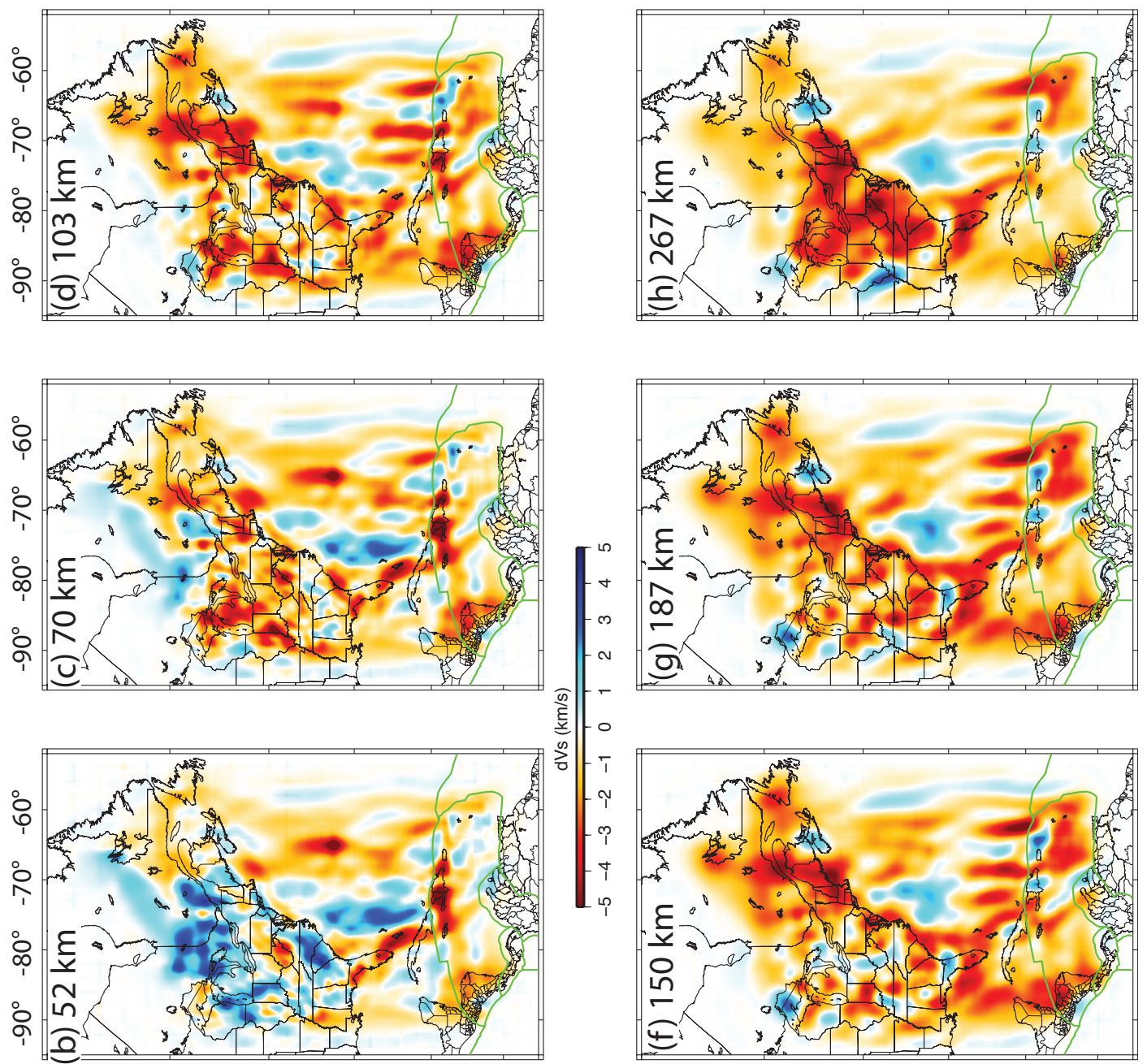

8
0
0
0
0
0
0
0
0
0
0
0
3
3

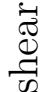

.尹

章

. 2

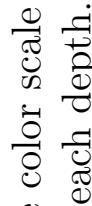

学

ن ह

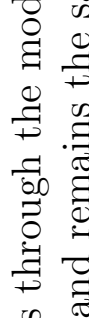
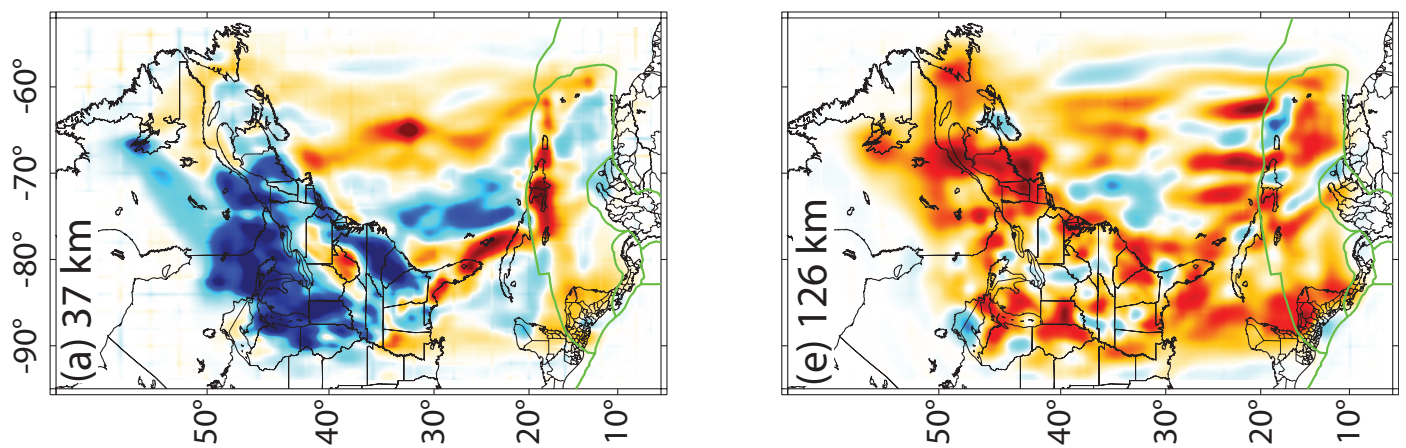

总

ن

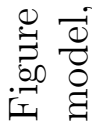



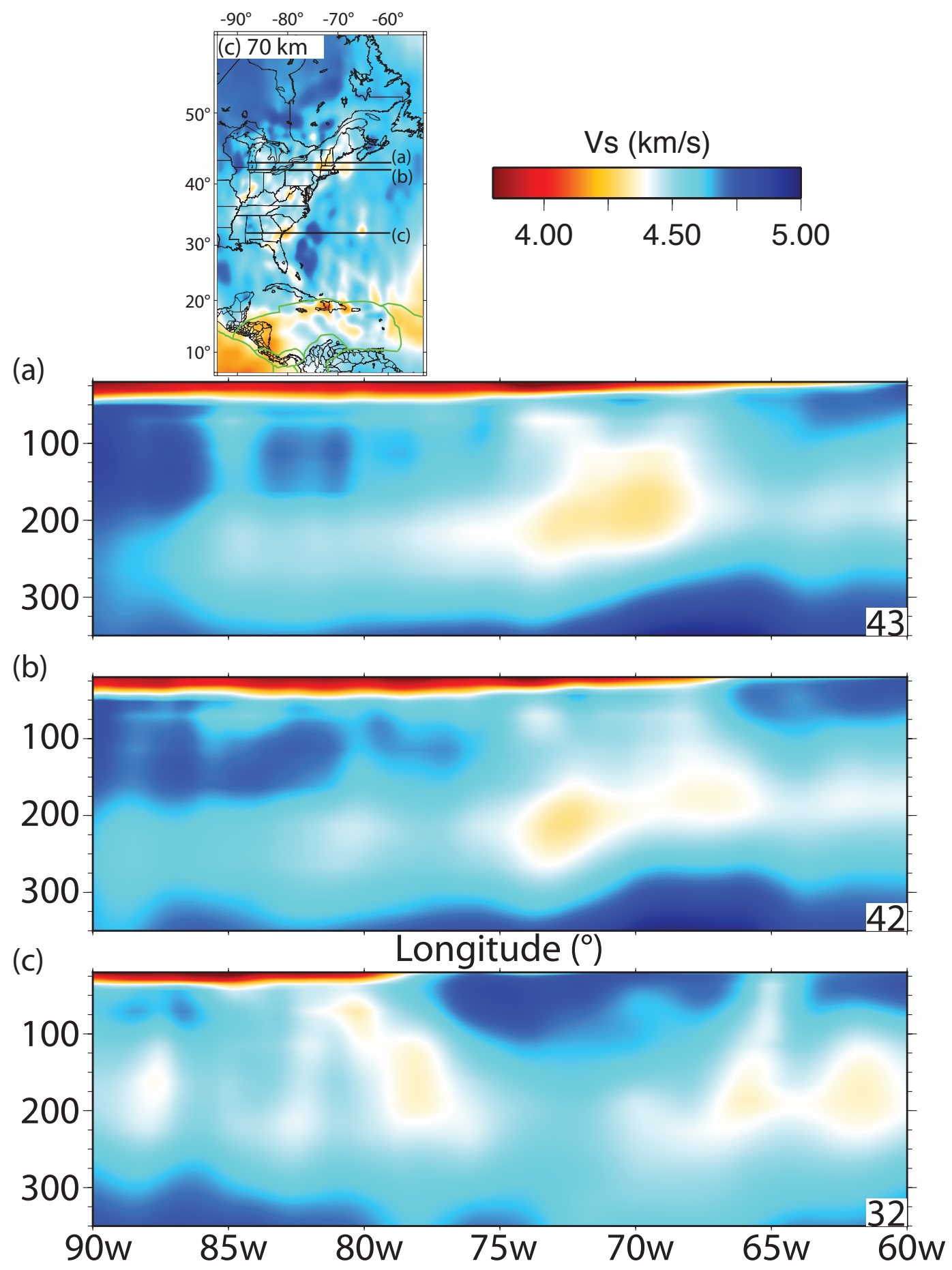

Figure 3.6: (a-b) Cross section through the New England low wave speed region. A seaward shallowing low wave speed anomaly is seen between depths of approximately $50-250 \mathrm{~km}$. (c) Cross section through the low wave speed anomaly offshore of South Carolina and Georgia. A seaward dipping low wave speed anomaly is seen between depths of approximately 35 to greater than $200 \mathrm{~km}$. 

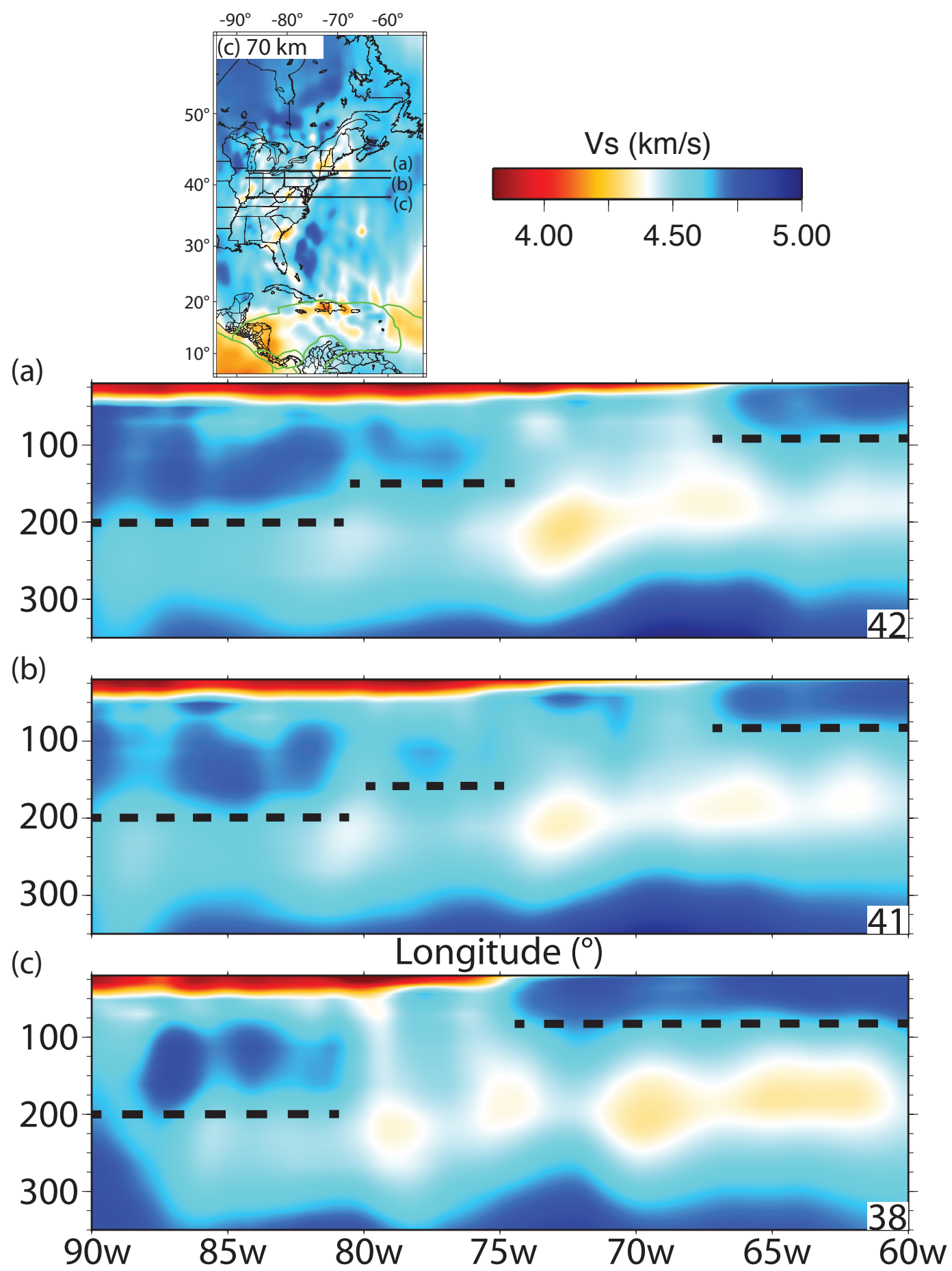

Figure 3.7: (a-c) Cross section showing the transition between oceanic and continental lithosphere thickness at three locations up the eastern North American margin. The approximate lithosphere asthenosphere boundary is indicated by the dashed black line. A gap in between the thick continental lithosphere and the much thinner oceanic lithosphere is aligned with low wave speed features possibly imaging edge-driven convection cells on the boundary of the continent. 


\section{BIBLIOGRAPHY}

Anderson, D. L., T. Tanimoto, and Y.-s. Zhang, Plate tectonics and hotspots: the third dimension, Science, 256(5064), 1645-1651, 1992.

Austin, J. A., P. L. Stoffa, J. D. Phillips, J. Oh, D. S. Sawyer, G. M. Purdy, E. Reiter, and J. Makris, Crustal structure of the southeast georgia embaymentcarolina trough: Preliminary results of a composite seismic image of a continental suture (?) and a volcanic passive margin, Geology, 18(10), 1023-1027, 1990.

Beutel, E. K., Magmatic rifting of pangaea linked to onset of south american plate motion, Tectonophysics, 468(1), 149-157, 2009.

Bijwaard, H., and W. Spakman, Non-linear global p-wave tomography by iterated linearized inversion, Geophysical Journal International, 141(1), 71-82, 2000.

Chen, P., T. H. Jordan, and L. Zhao, Full three-dimensional tomography: a comparison between the scattering-integral and adjoint-wavefield methods, Geophysical Journal International, 170(1), 175-181, 2007a.

Chen, P., L. Zhao, and T. H. Jordan, Full 3d tomography for the crustal structure of the los angeles region, Bulletin of the Seismological Society of America, 97(4), 1094-1120, 2007b.

Ekström, G., Love and Rayleigh phase-velocity maps, 5-40 s, of the western and central USA from USArray data, Earth and Planetary Science Letters, doi:http: //dx.doi.org/10.1016/j.epsl.2013.11.022, 2014.

Gao, H., and Y. Shen, Upper mantle structure of the cascades from full-wave ambient noise tomography: Evidence for 3d mantle upwelling in the back-arc, Earth and Planetary Science Letters, 390, 222-233, 2014. 
Gibert, B., U. Seipold, A. Tommasi, and D. Mainprice, Thermal diffusivity of upper mantle rocks: Influence of temperature, pressure, and the deformation fabric, Journal of Geophysical Research: Solid Earth (1978-2012), 108(B8), 2003.

Godey, S., R. Snieder, A. Villaseñor, and H. M. Benz, Surface wave tomography of north america and the caribbean using global and regional broad-band networks: phase velocity maps and limitations of ray theory, Geophysical Journal International, 152(3), 620-632, 2003.

Goes, S., and S. van der Lee, Thermal structure of the north american uppermost mantle inferred from seismic tomography, Journal of Geophysical Research: Solid Earth (1978-2012), 107(B3), ETG-2, 2002.

Grand, S. P., Mantle shear-wave tomography and the fate of subducted slabs, Philosophical Transactions of the Royal Society of London. Series A: Mathematical, Physical and Engineering Sciences, 360(1800), 2475-2491, 2002.

Gurnis, M., Large-scale mantle convection and the aggregation and dispersal of supercontinents, Nature, 332(6166), 695-699, 1988.

Hawman, R. B., Crustal thickness variations across the blue ridge mountains, southern appalachians: An alternative procedure for migrating wide-angle reflection data, Bulletin of the Seismological Society of America, 98(1), 469-475, 2008.

Hoffman, P. F., United plates of america, the birth of a craton-early proterozoic assembly and growth of laurentia, Annual Review of Earth and Planetary Sciences, $16,543-603,1988$.

Hoffman, P. F., Did the breakout of laurentia turn gondwanaland inside-out, Science, 252(5011), 1409-1412, 1991. 
Holbrook, W., and P. Kelemen, Large igneous province on the us atlantic margin and implications for magmatism during continental breakup, Nature, 364(6436), 433-436, 1993.

Holbrook, W. S., E. Reiter, G. Purdy, D. Sawyer, P. Stoffa, J. Austin, J. Oh, and J. Makris, Deep structure of the us atlantic continental margin, offshore south carolina, from coincident ocean bottom and multichannel seismic data, Journal of Geophysical Research: Solid Earth (1978-2012), 99(B5), 9155-9178, 1994.

Janney, P. E., and P. R. Castillo, Geochemistry of the oldest atlantic oceanic crust suggests mantle plume involvement in the early history of the central atlantic ocean, Earth and Planetary Science Letters, 192(3), 291-302, 2001.

Kennett, B., E. Engdahl, and R. Buland, Constraints on seismic velocities in the earth from traveltimes, Geophysical Journal International, 122(1), 108-124, 1995.

Levin, V., W. Menke, and J. Park, No regional anisotropic domains in the northeastern us appalachians, Journal of Geophysical Research: Solid Earth (19782012), 105 (B8), 19,029-19,042, 2000.

Li, A., K. M. Fischer, S. van der Lee, and M. E. Wysession, Crust and upper mantle discontinuity structure beneath eastern north america, Journal of Geophysical Research, 107(B5), 2100, 2002.

Li, A., D. W. Forsyth, and K. M. Fischer, Shear velocity structure and azimuthal anisotropy beneath eastern north america from rayleigh wave inversion, Journal of Geophysical Research: Solid Earth (1978-2012), 108(B8), 2003.

Liang, C., and C. A. Langston, Three-dimensional crustal structure of eastern north america extracted from ambient noise, Journal of Geophysical Research: Solid Earth (1978-2012), 114(B3), 2009. 
Lizarralde, D., J. B. Gaherty, J. A. Collins, G. Hirth, and S. D. Kim, Spreadingrate dependence of melt extraction at mid-ocean ridges from mantle seismic refraction data, Nature, 432(7018), 744-747, 2004.

Lizarralde, D., et al., Variation in styles of rifting in the gulf of california, Nature, 448(7152), 466-469, 2007.

McHone, J. G., Non-plume magmatism and rifting during the opening of the central atlantic ocean, Tectonophysics, 316(3), 287-296, 2000.

Menke, W., and V. Levin, Anomalous seaward dip of the lithosphere-asthenosphere boundary beneath northeastern usa detected using differential-array measurements of rayleigh waves, Geophysical Journal International, 149(2), 413-421, 2002.

Nomade, S., K. Knight, E. Beutel, P. Renne, C. Verati, G. Féraud, A. Marzoli, N. Youbi, and H. Bertrand, Chronology of the central atlantic magmatic province: implications for the central atlantic rifting processes and the triassicjurassic biotic crisis, Palaeogeography, Palaeoclimatology, Palaeoecology, 244(1), 326-344, 2007.

Oyarzun, R., M. Doblas, J. López-Ruiz, and J. M. Cebrá, Opening of the central atlantic and asymmetric mantle upwelling phenomena: implications for longlived magmatism in western north africa and europe, Geology, 25(8), 727-730, 1997.

Parker, E. H., R. B. Hawman, K. M. Fischer, and L. S. Wagner, Crustal evolution across the southern appalachians: Initial results from the sesame broadband array, Geophysical Research Letters, 40(15), 3853-3857, 2013.

Puffer, J. H., A reactivated back-arc source for camp magma, Geophysical Monograph Series, 136, 151-162, 2003. 
Ritzwoller, M. H., N. M. Shapiro, M. P. Barmin, and A. L. Levshin, Global surface wave diffraction tomography, Journal of Geophysical Research: Solid Earth (1978-2012), 107(B12), ESE-4, 2002.

Rivers, T., J. Martignole, C. Gower, and A. Davidson, New tectonic divisions of the grenville province, southeast canadian shield, Tectonics, 8(1), 63-84, 1989.

Rychert, C. A., K. M. Fischer, and S. Rondenay, A sharp lithosphereasthenosphere boundary imaged beneath eastern north america, Nature, 436(7050), 542-545, 2005.

Rychert, C. A., S. Rondenay, and K. M. Fischer, P-to-s and s-to-p imaging of a sharp lithosphere-asthenosphere boundary beneath eastern north america, Journal of Geophysical Research: Solid Earth (1978-2012), 112(B8), 2007.

Schlische, R. W., Structural geology, basin evolution, and tectonic history of the eastern north american rift system, The Great Rift Valleys of Pangea in Eastern North America: tectonics, structure, and volcanism, 1, 21, 2003.

Shen, Y., Y. Ren, H. Gao, and B. Savage, An improved method to extract verybroadband empirical green's functions from ambient seismic noise, Bulletin of the Seismological Society of America, 102(4), 1872-1877, 2012.

Thomas, W. A., Tectonic inheritance at a continental margin, GSA Today, 16(2), 4-11, 2006.

Till, C. B., L. T. Elkins-Tanton, and K. M. Fischer, A mechanism for low-extent melts at the lithosphere-asthenosphere boundary, Geochemistry, Geophysics, Geosystems, 11(10), 2010.

Van der Lee, S., and A. Frederiksen, Surface wave tomography applied to the north american upper mantle, Geophysical Monograph Series, 157, 67-80, 2005. 
Van der Lee, S., and G. Nolet, Upper mantle s velocity structure of north america, Journal of Geophysical Research, 102(B10), 22,815-22, 1997.

White, R., and D. McKenzie, Magmatism at rift zones: the generation of volcanic continental margins and flood basalts, Journal of Geophysical Research: Solid Earth (1978-2012), 94(B6), 7685-7729, 1989.

White, R. S., G. D. Spence, S. R. Fowler, D. P. McKenzie, and G. K. Westbrook, Magmatism at rifted continental margins, Nature, 330, 439-444, 1987.

Wilson, M., Thermal evolution of the central atlantic passive margins: continental break-up above a mesozoic super-plume, Journal of the Geological Society, $154(3), 491-495,1997$.

Zhang, W., Y. Shen, and L. Zhao, Three-dimensional anisotropic seismic wave modelling in spherical coordinates by a collocated-grid finite-difference method, Geophysical Journal International, 188(3), 1359-1381, 2012.

Zhang, Z., and Y. Shen, Cross-dependence of finite-frequency compressional waveforms to shear seismic wave speeds, Geophysical Journal International, 174(3), 941-948, 2008.

Zhang, Z., Y. Shen, and L. Zhao, Finite-frequency sensitivity kernels for head waves, Geophysical Journal International, 171(2), 847-856, 2007.

Zhao, L., T. Jordan, K. Olsen, and P. Chen, Fréchet kernels for imaging regional earth structure based on three-dimensional reference models, Bulletin of the Seismological Society of America, 95(6), 2066, 2005. 


\section{BIBLIOGRAPHY}

Adams, A., R. Brazier, A. Nyblade, A. Rodgers, and A. Al-Amri, Source Parameters for Moderate Earthquakes in the Zagros Mountains with Implications for the Depth Extent of Seismicity, Bulletin of the Seismological Society of America, $99(3), 2044,2009$.

Anderson, D. L., T. Tanimoto, and Y.-s. Zhang, Plate tectonics and hotspots: the third dimension, Science, 256(5064), 1645-1651, 1992.

Austin, J. A., P. L. Stoffa, J. D. Phillips, J. Oh, D. S. Sawyer, G. M. Purdy, E. Reiter, and J. Makris, Crustal structure of the southeast georgia embaymentcarolina trough: Preliminary results of a composite seismic image of a continental suture (?) and a volcanic passive margin, Geology, 18(10), 1023-1027, 1990.

Bascou, J., G. Barruol, A. Vauchez, D. Mainprice, and M. Egydio-Silva, Ebsdmeasured lattice-preferred orientations and seismic properties of eclogites, Tectonophysics, 342(1), 61-80, 2001.

Bassin, C., G. Laske, and G. Masters, The current limits of resolution for surface wave tomography in north america, EOS Trans. AGU., 81(F897), 2000.

Becker, T., On recent seismic tomography for the western united states, Geochemistry Geophysics Geosystems, 13, 2012.

Beghein, C., K. Yuan, N. Schmerr, and Z. Xing, Changes in seismic anisotropy shed light on the nature of the gutenberg discontinuity., Science (New York, NY), 2014.

Bercovici, D., and J. Mahoney, Double flood basalts and plume head separation at the 660-kilometer discontinuity, Science, 266(5189), 1367-1369, 1994. 
Beutel, E. K., Magmatic rifting of pangaea linked to onset of south american plate motion, Tectonophysics, 468(1), 149-157, 2009.

Bijwaard, H., and W. Spakman, Non-linear global p-wave tomography by iterated linearized inversion, Geophysical Journal International, 141(1), 71-82, 2000.

Billen, M. I., and J. Stock, Morphology and origin of the osbourn trough, Journal of Geophysical Research: Solid Earth (1978-2012), 105(B6), 13,481-13,489, 2000.

Bird, P., An updated digital model of plate boundaries, Geochemistry, Geophysics, Geosystems, 4(3), 2003.

Cammarano, F., and B. Romanowicz, Insights into the nature of the transition zone from physically constrained inversion of long-period seismic data, Proceedings of the National Academy of Sciences, 104(22), 9139-9144, 2007.

Campbell, I., The Earth's Mantle: Composition, Structure and Evolution, chap. The mantle's chemical structure: insights from the melting products of mantle plumes, pp. 259-310, 1998.

Campbell, I. H., Large igneous provinces and the mantle plume hypothesis, Elements, $1(5), 265-269,2005$.

Chandler, M. T., P. Wessel, B. Taylor, M. Seton, S.-S. Kim, and K. Hyeong, Reconstructing ontong java nui: Implications for pacific absolute plate motion, hotspot drift and true polar wander, Earth and Planetary Science Letters, 331, 140-151, 2012.

Chen, P., T. H. Jordan, and L. Zhao, Full three-dimensional tomography: a comparison between the scattering-integral and adjoint-wavefield methods, Geophysical Journal International, 170(1), 175-181, 2007a. 
Chen, P., L. Zhao, and T. H. Jordan, Full 3d tomography for the crustal structure of the los angeles region, Bulletin of the Seismological Society of America, 97(4), 1094-1120, 2007b.

Coffin, M., and O. Eldholm, Large igneous provinces: crustal structure, dimensions, and external consequences, Reviews of Geophysics, 32, 1-1, 1994.

Covellone, B. M., and B. Savage, A quantitative comparison between $1 \mathrm{~d}$ and $3 \mathrm{~d}$ source inversion methodologies: Application to the middle east, Bulletin of the Seismological Society of America, 102(5), 2189-2199, 2012.

Crisp, J. A., Rates of magma emplacement and volcanic output, Journal of Volcanology and Geothermal Research, 20(3), 177-211, 1984.

Dalton, C. A., G. Ekström, and A. M. Dziewonski, Global seismological shear velocity and attenuation: A comparison with experimental observations, Earth and Planetary Science Letters, 284(1), 65-75, 2009.

DeMets, C., R. Gordon, D. Argus, and S. Stein, Effect of recent revisions to the geomagnetic reversal rimescale on estimates of current plate motions, Geophysical Research Letters, 21, 2191-2194, 1994.

Druken, K., C. Kincaid, and R. Griffiths, Directions of seismic anisotropy in laboratory models of mantle plumes, Geophysical Research Letters, 40(14), 3544-3549, 2013.

Druken, K., C. Kincaid, R. Griffiths, D. Stegman, and S. Hart, Plume-slab interaction: The samoa-tonga system, Physics of the Earth and Planetary Interiors, 2014.

Dziewonski, A., and D. Anderson, Preliminary reference Earth model, Physics of the Earth and Planetary Interiors, 25(4), 297-356, 1981. 
Dziewonski, A., T. Chou, and J. Woodhouse, Determination of earthquake source parameters from waveform data for studies of global and regional seismicity, Journal of Geophysical Research, 86(B4), 2825-2852, 1981.

Ely, J., and C. Neal, Using platinum-group elements to investigate the origin of the ontong java plateau, sw pacific, Chemical Geology, 196(1-4), 235-257, 2003.

Farnetani, C., and M. Richards, Numerical investigations of the mantle plume initiation model for flood basalt events, Journal of Geophysical Research, 99, 13-13, 1994.

Fischer, K. M., H. A. Ford, D. L. Abt, and C. A. Rychert, The lithosphereasthenosphere boundary, Annual Review of Earth and Planetary Sciences, 38, $551-575,2010$.

Furumoto, A. S., J. P. Webb, M. E. Odegard, and D. M. Hussong, Seismic studies on the ontong java plateau, 1970, Tectonophysics, 34(1), 71-90, 1976.

Gao, H., and Y. Shen, Validation of shear-wave velocity models of the pacific northwest, Bulletin of the Seismological Society of America, 102(6), 2611-2621, 2012.

Gao, H., and Y. Shen, Upper mantle structure of the cascades from full-wave ambient noise tomography: Evidence for 3d mantle upwelling in the back-arc, Earth and Planetary Science Letters, 390, 222-233, 2014.

Gee, L. S., and T. H. Jordan, Generalized seismological data functionals, Geophysical Journal International, 111(2), 363-390, 1992.

Gladczenko, T., M. Coffin, and O. Eldholm, Crustal structure of the ontong java plateau: modeling of new gravity and existing seismic data, Journal of Geophysical Research, 102, 22-22, 1997. 
Godey, S., R. Snieder, A. Villaseñor, and H. M. Benz, Surface wave tomography of north america and the caribbean using global and regional broad-band networks: phase velocity maps and limitations of ray theory, Geophysical Journal International, 152(3), 620-632, 2003.

Goes, S., and S. van der Lee, Thermal structure of the north american uppermost mantle inferred from seismic tomography, Journal of Geophysical Research: Solid Earth (1978-2012), 107(B3), ETG-2, 2002.

Gomer, B., and E. Okal, Multiple-scs probing of the ontong-java plateau, Physics of the Earth and Planetary Interiors, 138(3), 317-331, 2003.

Grand, S. P., and D. V. Helmberger, Upper mantle shear structure of north america, Geophysical Journal International, 76(2), 399-438, 1984.

Griffiths, R., M. Gurnis, and G. Eitelberg, Holographic measurements of surface topography in laboratory models of mantle hotspots, Geophysical Journal International, 96(3), 477-495, 1989.

Gurnis, M., Large-scale mantle convection and the aggregation and dispersal of supercontinents, Nature, 332(6166), 695-699, 1988.

Hacker, B. R., and G. A. Abers, Subduction factory 3: An excel worksheet and macro for calculating the densities, seismic wave speeds, and h2o contents of minerals and rocks at pressure and temperature, Geochemistry, Geophysics, Geosystems, 5(1), 2004.

Hall, P. S., and C. Kincaid, Melting, dehydration, and the geochemistry of off-axis plume-ridge interaction, Geochemistry, Geophysics, Geosystems, 5(12), 2004.

Hammond, W., and E. Humphreys, Upper mantle seismic wave velocity: Effects of realistic partial melt geometries, Jour. Geophys. Res., 105(B5), 2000. 
Hatzfeld, D., and P. Molnar, Comparisons of the kinematics and deep structures of the Zagros and Himalaya and of the Iranian and Tibetan plateaus and geodynamic implications, Reviews of Geophysics, 48(2), 2010.

Henry, C., J. Woodhouse, and S. Das, Stability of earthquake moment tensor inversions: effect of the double-couple constraint, Tectonophysics, 356(1-3), 115$124,2002$.

Hjörleifsdóttir, V., and G. Ekström, Effects of three-dimensional earth structure on cmt earthquake parameters, Physics of the Earth and Planetary Interiors, $179(3-4), 178-190,2010$.

Hoffman, P. F., United plates of america, the birth of a craton-early proterozoic assembly and growth of laurentia, Annual Review of Earth and Planetary Sciences, 16, 543-603, 1988.

Hoffman, P. F., Did the breakout of laurentia turn gondwanaland inside-out, Science, 252(5011), 1409-1412, 1991.

Holbrook, W., and P. Kelemen, Large igneous province on the us atlantic margin and implications for magmatism during continental breakup, Nature, 364(6436), 433-436, 1993.

Holbrook, W. S., E. Reiter, G. Purdy, D. Sawyer, P. Stoffa, J. Austin, J. Oh, and J. Makris, Deep structure of the us atlantic continental margin, offshore south carolina, from coincident ocean bottom and multichannel seismic data, Journal of Geophysical Research: Solid Earth (1978-2012), 99(B5), 9155-9178, 1994.

Hollingsworth, J., J. Jackson, R. Walker, M. Gheitanchi, and M. Bolourchi, Strikeslip faulting, rotation, and along-strike elongation in the Kopeh Dagh mountains, NE Iran, Geophysical Journal International, $166(3)$, 1161-1177, 2006. 
Hollingsworth, J., J. Jackson, R. Walker, and H. Nazari, Extrusion tectonics and subduction in the eastern South Caspian region since 10 Ma, Geology, 36(10), 763, 2008.

Hollingsworth, J., J. Jackson, R. Walker, and H. Nazari, Extrusion tectonics and subduction in the eastern South Caspian region since 10 Ma: Reply, Geology, 37(12), e199, 2009.

Holt, W., J. Ni, T. Wallace, and A. Haines, The active tectonics of the eastern Himalayan syntaxis and surrounding regions, Journal of Geophysical ResearchSolid Earth, 96 (B9), 14,595-14,632, 1991.

Hung, S., Y. Shen, and L. Chiao, Imaging seismic velocity structure beneath the iceland hot spot: A finite frequency approach, Journal of geophysical research, 109 (B8), B08,305, 2004.

Hussong, D., L. Wipperman, and L. Kroenke, The crustal structure of the ontong java and manihiki oceanic plateaus, Journal of Geophysical Research, 84(B11), 6003-6010, 1979.

Ishikawa, A., S. Maruyama, and T. Komiya, Layered lithospheric mantle beneath the ontong java plateau: implications from xenoliths in alnöite, malaita, solomon islands, Journal of Petrology, 45(10), 2011-2044, 2004.

Ishikawa, A., T. Kuritani, A. Makishima, and E. Nakamura, Ancient recycled crust beneath the ontong java plateau: Isotopic evidence from the garnet clinopyroxenite xenoliths, malaita, solomon islands, Earth and Planetary Science Letters, 259(1), 134-148, 2007.

Ito, G., and A. Taira, Compensation of the ontong java plateau by surface and subsurface loading, Journal of geophysical research, 105(11), 171-11, 2000. 
Ito, G., and P. van Keken, Hotspots and melting anomalies, Treatise on Geophysics, 7, 371-436, 2007.

Jackson, J., K. Priestley, M. Allen, and M. Berberian, Active tectonics of the South Caspian basin, Geophysical Journal International, 148(2), 214-245, 2002.

Jain, J., C. Neal, and J. O'Neill Jr, Origin of the ontong java plateau (ojp) at the core-mantle boundary: platinum group element (pge) and gold (au) evidence, Transactions of the American Geophysical Union, EOS, 77, 714, 1996.

Janney, P. E., and P. R. Castillo, Geochemistry of the oldest atlantic oceanic crust suggests mantle plume involvement in the early history of the central atlantic ocean, Earth and Planetary Science Letters, 192(3), 291-302, 2001.

Ji, S., K. Saruwatari, D. Mainprice, R. Wirth, Z. Xu, and B. Xia, Microstructures, petrofabrics and seismic properties of ultra high-pressure eclogites from sulu region, china: implications for rheology of subducted continental crust and origin of mantle reflections, Tectonophysics, 370(1), 49-76, 2003.

Julian, B., A. Miller, and G. Foulger, Non-double-couple earthquakes 1. theory, Reviews of Geophysics, 36(4), 525-549, 1998.

Karato, S., and H. Jung, Water, partial melting and the origin of the seismic low velocity and high attenuation zone in the upper mantle, Earth and Planetary Science Letters, 157(3-4), 193-207, 1998.

Kennett, B., E. Engdahl, and R. Buland, Constraints on seismic velocities in the earth from traveltimes, Geophysical Journal International, 122(1), 108-124, 1995.

Kincaid, C., K. Druken, R. Griffiths, and D. Stegman, Bifurcation of the yellow- 
stone plume driven by subduction-induced mantle flow, Nature Geoscience, 6(5), 395-399, 2013.

Klosko, E., R. Russo, E. Okal, and W. Richardson, Evidence for a rheologically strong chemical mantle root beneath the ontong-java plateau, Earth and Planetary Science Letters, 186(3), 347-361, 2001.

Komatitsch, D., and J. Tromp, Introduction to the spectral element method for three-dimensional seismic wave propagation, Geophysical Jounrnal International, 139(3), 806-822, 1999.

Komatitsch, D., and J. Tromp, Spectral-element simulations of global seismic wave propagation-I. Validation, Geophysical Journal International, 149(2), 390-412, 2002a.

Komatitsch, D., and J. Tromp, Spectral-element simulations of global seismic wave propagation-II. Three-dimensional models, oceans, rotation and self-gravitation, Geophysical Journal International, 150(1), 303-318, 2002 b.

Korenaga, J., Why did not the ontong java plateau form subaerially?, Earth and Planetary Science Letters, 234(3-4), 385-399, 2005.

Kustowski, B., G. Ekström, and A. Dziewoński, Anisotropic shear-wave velocity structure of the earth's mantle: A global model, Journal of Geophysical Research: Solid Earth (1978-2012), 113(B6), 2008a.

Kustowski, B., G. Ekstrom, and A. Dziewonski, The shear-wave velocity structure in the upper mantle beneath Eurasia, Geophysical Journal International, $174(3)$, 978-992, 2008b.

Larson, R., Latest pulse of earth: Evidence for a mid-cretaceous superplume, $G e$ ology, $19(6), 547-550,1991$. 
Larson, R., Superplumes and ridge interactions between ontong java and manihiki plateaus and the nova-canton trough, Geology, 25(9), 779-782, 1997.

Larson, R. L., and C. Kincaid, Onset of mid-cretaceous volcanism by elevation of the $670 \mathrm{~km}$ thermal boundary layer, Geology, 24(6), 551-554, 1996.

Levin, V., W. Menke, and J. Park, No regional anisotropic domains in the northeastern us appalachians, Journal of Geophysical Research: Solid Earth (19782012), 105 (B8), 19,029-19,042, 2000.

Li, A., K. M. Fischer, S. van der Lee, and M. E. Wysession, Crust and upper mantle discontinuity structure beneath eastern north america, Journal of Geophysical Research, 107(B5), 2100, 2002.

Li, A., D. W. Forsyth, and K. M. Fischer, Shear velocity structure and azimuthal anisotropy beneath eastern north america from rayleigh wave inversion, Journal of Geophysical Research: Solid Earth (1978-2012), 108(B8), 2003.

Liang, C., and C. A. Langston, Three-dimensional crustal structure of eastern north america extracted from ambient noise, Journal of Geophysical Research: Solid Earth (1978-2012), 114(B3), 2009.

Liu, Q., J. Polet, D. Komatitsch, and J. Tromp, Spectral-element moment tensor inversions for earthquakes in southern California, Bulletin of the Seismologiacl Society of America, 94(5), 1748-1761, 2004.

Lizarralde, D., J. B. Gaherty, J. A. Collins, G. Hirth, and S. D. Kim, Spreadingrate dependence of melt extraction at mid-ocean ridges from mantle seismic refraction data, Nature, 432(7018), 744-747, 2004.

Maggi, A., E. Debayle, K. Priestley, and G. Barruol, Multimode surface wave- 
form tomography of the pacific ocean: a closer look at the lithospheric cooling signature, Geophysical Journal International, 166(3), 1384-1397, 2006.

Maggi, A., C. Tape, M. Chen, D. Chao, and J. Tromp, An automated time-window selection algorithm for seismic tomography, Geophysical Journal International, $178(1), 2009$.

Mahoney, J., J. Fitton, and P. Wallace, Initial reports, in Proceedings of the Ocean Drilling Program, vol. 192, 2001.

McHone, J. G., Non-plume magmatism and rifting during the opening of the central atlantic ocean, Tectonophysics, 316(3), 287-296, 2000.

Menke, W., and V. Levin, Anomalous seaward dip of the lithosphere-asthenosphere boundary beneath northeastern usa detected using differential-array measurements of rayleigh waves, Geophysical Journal International, 149(2), 413-421, 2002.

Michael, P., Implications for magmatic processes at ontong java plateau from volatile and major element contents of cretaceous basalt glasses, Geochemistry Geophysics Geosystems, 1(12), 1008-17, 1999.

Miller, A., G. Foulger, and B. Julian, Non-double-couple earthquakes. 2. Observations, Reviews of Geophysics, 36(4), 551-568, 1998.

Müller, R. D., M. Sdrolias, C. Gaina, and W. R. Roest, Age, spreading rates, and spreading asymmetry of the world's ocean crust, Geochemistry, Geophysics, Geosystems, 9(4), 2008.

Muyzert, E., and R. Snieder, The influence of errors in source parameters on phase velocity measurements of surface waves, Bulletin of the Seismological Society of America, 86(6), 1863-1872, 1996. 
Nakanishi, M., K. Tamaki, and K. Kobayashi, Magnetic anomaly lineations from late jurassic to early cretaceous in the west-central pacific ocean, Geophysical Journal International, 109(3), 701-719, 1992.

Neal, C., J. Mahoney, L. Kroenke, R. Duncan, and M. Petterson, Large Igneous Provinces: Continental, Oceanic, and Planetary Flood Volcanism, vol. 100, chap. The Ontong Java Plateau, pp. 183-216, 1997.

NOAA, Data Announcement 88-MGG-02. Digital relief of the surface of the Earth, National Oceanographic and Atmospheric Administration, National Geophysical Data Center, Boulder, Colorado, 1988.

Nomade, S., K. Knight, E. Beutel, P. Renne, C. Verati, G. Féraud, A. Marzoli, N. Youbi, and H. Bertrand, Chronology of the central atlantic magmatic province: implications for the central atlantic rifting processes and the triassicjurassic biotic crisis, Palaeogeography, Palaeoclimatology, Palaeoecology, $244(1)$, 326-344, 2007.

Olson, P., Hot spots, swells and mantle plumes, Magma transport and storage, pp. 33-51, 1990.

Oyarzun, R., M. Doblas, J. López-Ruiz, and J. M. Cebrá, Opening of the central atlantic and asymmetric mantle upwelling phenomena: implications for longlived magmatism in western north africa and europe, Geology, 25(8), 727-730, 1997.

Panning, M., V. Lekić, and B. Romanowicz, Importance of crustal corrections in the development of a new global model of radial anisotropy, Journal of Geophysical Research: Solid Earth (1978-2012), 115(B12), 2010.

Parker, E. H., R. B. Hawman, K. M. Fischer, and L. S. Wagner, Crustal evolution 
across the southern appalachians: Initial results from the sesame broadband array, Geophysical Research Letters, 40(15), 3853-3857, 2013.

Press, W., S. Teukolsky, W. T. Vetterling, and B. P. Flannery, Numerical Recipes in C, 2nd ed., Cambridge University Press, 1997.

Puffer, J. H., A reactivated back-arc source for camp magma, Geophysical Monograph Series, 136, 151-162, 2003.

Reigber, C., et al., New space geodetic constraints on the distribution of deformation in Central Asia, Earth and Planetary Science Letters, 191(1-2), 157-165, 2001.

Ren, Y., and Y. Shen, Finite frequency tomography in southeastern tibet: evidence for the causal relationship between mantle lithosphere delamination and the north-south trending rifts, Journal of Geophysical Research: Solid Earth (19782012), 113(B10), 2008.

Richardson, W., E. Okal, and S. Van der Lee, Rayleigh-wave tomography of the ontong-java plateau, Physics of the Earth and Planetary Interiors, 118(1-2), $29-51,2000$.

Ritsema, J., A. Deuss, H. Van Heijst, and J. Woodhouse, S40rts: a degree-40 shear-velocity model for the mantle from new rayleigh wave dispersion, teleseismic traveltime and normal-mode splitting function measurements, Geophysical Journal International, 184(3), 1223-1236, 2011.

Ritzwoller, M. H., N. M. Shapiro, M. P. Barmin, and A. L. Levshin, Global surface wave diffraction tomography, Journal of Geophysical Research: Solid Earth (1978-2012), 107(B12), ESE-4, 2002. 
Rivers, T., J. Martignole, C. Gower, and A. Davidson, New tectonic divisions of the grenville province, southeast canadian shield, Tectonics, 8(1), 63-84, 1989.

Romanowicz, B., The thickness of tectonic plates, Science, 324(5926), 474-476, 2009.

Rychert, C. A., K. M. Fischer, and S. Rondenay, A sharp lithosphereasthenosphere boundary imaged beneath eastern north america, Nature, $436(7050), 542-545,2005$.

Rychert, C. A., S. Rondenay, and K. M. Fischer, P-to-s and s-to-p imaging of a sharp lithosphere-asthenosphere boundary beneath eastern north america, Journal of Geophysical Research: Solid Earth (1978-2012), 112(B8), 2007.

Savage, B., D. Peter, B. M. Covellone, C. Morency, A. Rodgers, and J. Tromp, Short-period, anelastic, and anisotropic, waveform-based 3d middle east model to improve nuclear explosion monitoring, in Proceedings: 34th Monitoring Research Review (MRR 2012), 2012.

Schlische, R. W., Structural geology, basin evolution, and tectonic history of the eastern north american rift system, The Great Rift Valleys of Pangea in Eastern North America: tectonics, structure, and volcanism, 1, 21, 2003.

Shen, Y., and W. Zhang, Multi-grid and resolution full-wave tomography and moment tensor inversion, in Proceedings: 34th Monitoring Research Review (MRR 2012), 2012.

Shen, Y., Y. Ren, H. Gao, and B. Savage, An improved method to extract verybroadband empirical green's functions from ambient seismic noise, Bulletin of the Seismological Society of America, 102(4), 1872-1877, 2012. 
Silver, P. G., M. D. Behn, K. Kelley, M. Schmitz, and B. Savage, Understanding cratonic flood basalts, Earth and Planetary Science Letters, 245(1), 190-201, 2006.

Snyder, D. B., Stacked uppermost mantle layers within the slave craton of nw canada as defined by anisotropic seismic discontinuities, Tectonics, 27(4), 2008.

Sobolev, S., A. Sobolev, D. Kuzmin, N. Krivolutskaya, A. Petrunin, N. Arndt, V. Radko, and Y. Vasiliev, Linking mantle plumes, large igneous provinces and environmental catastrophes, Nature, 477(7364), 312-316, 2011.

Stein, C. A., and S. Stein, A model for the global variation in oceanic depth and heat flow with lithospheric age, Nature, 359(6391), 123-129, 1992.

Stein, S., and M. Wysession, An introduction to seismology, earthquakes, and earth structure, Wiley-Blackwell, 2009.

Talebian, M., and J. Jackson, A reappraisal of earthquake focal mechanisms and active shortening in the Zagros mountains of Iran, Geophysical Journal International, 156(3), 506-526, 2004.

Tape, C., Q. Liu, and J. Tromp, Finite-frequency tomography using adjoint methods - methodology and examples using membrane surface waves, Geophysical Journal International, 168(3), 1105-1129, 2007.

Tape, C., Q. Liu, A. Maggi, and J. Tromp, Adjoint tomography of the southern California crust, Science, 325(5943), 988, 2009.

Tatar, M., D. Hatzfeld, and M. Ghafory-Ashtiany, Tectonics of the Central Zagros (Iran) deduced from microearthquake seismicity, Geophysical Journal International, 156(2), 255-266, 2004. 
Taylor, B., The single largest oceanic plateau: Ontong java-manihiki-hikurangi, Earth and Planetary Science Letters, 241(3-4), 372-380, 2006.

Tejada, M., J. Mahoney, R. Duncan, and M. Hawkins, Age and geochemistry of basement and alkalic rocks of malaita and santa isabel, solomon islands, southern margin of ontong java plateau, Journal of Petrology, 37(2), 361-394, 1996.

Tejada, M., J. Mahoney, C. Neal, R. Duncan, and M. Petterson, Basement geochemistry and geochronology of central malaita, solomon islands, with implications for the origin and evolution of the ontong java plateau, Journal of Petrology, 43(3), 449-484, 2002.

Tejada, M., J. Mahoney, P. Castillo, S. Ingle, H. Sheth, and D. Weis, Pin-pricking the elephant: Evidence on the origin of the ontong java plateau from pb-sr-hfnd isotopic characteristics of odp leg 192 basalts, Geological Society, London, Special Publications, 229(1), 133-150, 2004.

Thomas, W. A., Tectonic inheritance at a continental margin, GSA Today, 16(2), 4-11, 2006.

Till, C. B., L. T. Elkins-Tanton, and K. M. Fischer, A mechanism for low-extent melts at the lithosphere-asthenosphere boundary, Geochemistry, Geophysics, Geosystems, 11(10), 2010.

Van der Lee, S., and A. Frederiksen, Surface wave tomography applied to the north american upper mantle, Geophysical Monograph Series, 157, 67-80, 2005.

Van der Lee, S., and G. Nolet, Upper mantle s velocity structure of north america, Journal of Geophysical Research, 102(B10), 22,815-22, 1997.

Vernant, P., et al., Present-day crustal deformation and plate kinematics in the 
Middle East constrained by GPS measurements in Iran and northern Oman, Geophysical Journal International, 157(1), 381-398, 2004.

Viso, R. F., R. L. Larson, and R. A. Pockalny, Tectonic evolution of the pacificphoenix-farallon triple junction in the south pacific ocean, Earth and Planetary Science Letters, 233(1), 179-194, 2005.

Wells, D. L., and K. J. Coppersmith, New empirical relationships among magnitude, rupture length, rupture width, rupture area, and surface displacement, Bulletin of the Seismological Society of America, 84(4), 974-1002, 1994.

Wessel, P., and W. Smith, Free software helps map and display data, Eos Trans. $A G U$, 72(441), 445-446, 1991.

White, R., and D. McKenzie, Magmatism at rift zones: the generation of volcanic continental margins and flood basalts, Journal of Geophysical Research: Solid Earth (1978-2012), 94(B6), 7685-7729, 1989.

White, R. S., G. D. Spence, S. R. Fowler, D. P. McKenzie, and G. K. Westbrook, Magmatism at rifted continental margins, Nature, 330, 439-444, 1987.

Wilson, M., Thermal evolution of the central atlantic passive margins: continental break-up above a mesozoic super-plume, Journal of the Geological Society, $154(3), 491-495,1997$.

Worthington, J. R., B. R. Hacker, and G. Zandt, Distinguishing eclogite from peridotite: Ebsd-based calculations of seismic velocities, Geophysical Journal International, 193(1), 489-505, 2013.

Zhang, J., Y. Wang, and Z. Jin, Cpo-induced seismic anisotropy in uhp eclogites, Science in China Series D: Earth Sciences, 51(1), 11-21, 2008. 
Zhang, W., Y. Shen, and L. Zhao, Three-dimensional anisotropic seismic wave modelling in spherical coordinates by a collocated-grid finite-difference method, Geophysical Journal International, 188(3), 1359-1381, 2012.

Zhang, Z., and Y. Shen, Cross-dependence of finite-frequency compressional waveforms to shear seismic wave speeds, Geophysical Journal International, 174(3), 941-948, 2008.

Zhang, Z., Y. Shen, and L. Zhao, Finite-frequency sensitivity kernels for head waves, Geophysical Journal International, 171(2), 847-856, 2007.

Zhao, L., T. Jordan, K. Olsen, and P. Chen, Fréchet kernels for imaging regional earth structure based on three-dimensional reference models, Bulletin of the Seismological Society of America, 95(6), 2066, 2005.

Zhu, H., and J. Tromp, Mapping tectonic deformation in the crust and upper mantle beneath europe and the north atlantic ocean, Science, 341(6148), 871$875,2013$.

Zhu, H., E. Bozdağ, D. Peter, and J. Tromp, Seismic wavespeed images across the iapetus and tornquist suture zones, Geophysical Research Letters, 39(18), 2012a.

Zhu, H., E. Bozdağ, D. Peter, and J. Tromp, Structure of the european upper mantle revealed by adjoint tomography, Nature Geoscience, 5(7), 493-498, 2012b.

Zhu, H., E. Bozdağ, T. S. Duffy, and J. Tromp, Seismic attenuation beneath europe and the north atlantic: Implications for water in the mantle, Earth and Planetary Science Letters, 381, 1-11, 2013. 